\title{
Exponential integrators
}

\author{
Marlis Hochbruck \\ Karlsruher Institut für Technologie, \\ Institut für Angewandte und Numerische Mathematik, \\ D-76128 Karlsruhe, \\ Germany \\ E-mail: marlis.hochbruck@kit.edu
}

\author{
Alexander Ostermann \\ Institut für Mathematik, \\ Universität Innsbruck, \\ A-6020 Innsbruck, \\ Austria
}

E-mail: alexander.ostermann@uibk.ac.at

In this paper we consider the construction, analysis, implementation and application of exponential integrators. The focus will be on two types of stiff problems. The first one is characterized by a Jacobian that possesses eigenvalues with large negative real parts. Parabolic partial differential equations and their spatial discretization are typical examples. The second class consists of highly oscillatory problems with purely imaginary eigenvalues of large modulus. Apart from motivating the construction of exponential integrators for various classes of problems, our main intention in this article is to present the mathematics behind these methods. We will derive error bounds that are independent of stiffness or highest frequencies in the system.

Since the implementation of exponential integrators requires the evaluation of the product of a matrix function with a vector, we will briefly discuss some possible approaches as well. The paper concludes with some applications, in which exponential integrators are used.

\section{CONTENTS}

1 Introduction 210

2 Parabolic problems, smooth solutions 213

3 Highly oscillatory problems 244

4 Implementation issues 264

5 Applications 273

6 Historical remarks 277

References $\quad 279$ 


\section{Introduction}

Exponential integrators constitute an interesting class of numerical methods for the time integration of stiff systems of differential equations, that is,

$$
u^{\prime}(t)=F(t, u(t)), \quad u(0)=u_{0} .
$$

In this survey article we will mainly consider two types of stiff problems. The first one is characterized by a Jacobian that possesses eigenvalues with large negative real parts. For these problems, the usual definition of stiffness applies which states that a differential equation is stiff whenever the implicit Euler method works (tremendously) better than the explicit Euler method. The reason for this behaviour lies in the different linear stability properties of the two methods. All available explicit integrators (with the exception of Runge-Kutta-Chebyshev methods) have a relatively small linear stability domain in the complex left half-plane, and this is the reason why explicit methods require unrealistically small step sizes for integrating stiff problems.

The second class of stiff problems considered in this survey consists of highly oscillatory problems with purely imaginary eigenvalues of large modulus. Again, explicit methods lack stability and are forced to use tiny time steps. For this class, however, the implicit Euler scheme does not perform well either. At first glance this behaviour is puzzling since the method has the required stability properties. A closer look reveals that the step size reduction is forced by accuracy requirements: the method tends to resolve all the oscillations in the solution, hence its numerical inefficiency.

The basic idea behind exponential integrators is to identify a prototypical differential equation that has stiffness properties similar to those of the underlying equation (1.1) and that can be solved exactly. This prototypical equation is often found by linearizing (1.1) at a certain state $w$. For autonomous systems, this yields

$$
v^{\prime}(t)+A v(t)=g(v(t)), \quad v(0)=u_{0}-w,
$$

with $A=-\mathrm{D} F(w)$ and $v(t)=u(t)-w$. This linearization procedure gives rise to a semilinear equation with a comparably small nonlinear remainder $g$, if $u(t)$ is close to $w$. The linear part of (1.2),

$$
v^{\prime}(t)+A v(t)=0, \quad v(0)=v_{0},
$$

can then serve as the prototypical equation with exact solution

$$
v(t)=\mathrm{e}^{-t A} v_{0} .
$$

If, for example, $A$ is symmetric positive definite or skew-Hermitian with eigenvalues of large modulus, the exponential $\mathrm{e}^{-t A}$ enjoys favourable properties such as uniform boundedness, independent of the time step $t$, in contrast to the propagator $I-t A$ of the explicit Euler method. For oscillatory 
problems, the exponential contains the full information on linear oscillations, in contrast to the propagator $(I+t A)^{-1}$ of the implicit Euler method.

The numerical scheme for the full equation (1.1) or (1.2) is constructed by incorporating the exact propagator of (1.3) in an appropriate way. In the above situation, this can be achieved by considering the corresponding Volterra integral equation

$$
u(t)=\mathrm{e}^{-t A} u_{0}+\int_{0}^{t} \mathrm{e}^{-(t-\tau) A} g(u(\tau)) \mathrm{d} \tau
$$

instead of (1.2). This representation of the exact solution is also called variation-of-constants formula.

The simplest numerical method for (1.5) is obtained by interpolating the nonlinearity at the known value $g\left(u_{0}\right)$ only, leading to the exponential Euler approximation

$$
u_{1}=\mathrm{e}^{-h A} u_{0}+h \varphi_{1}(-h A) g\left(u_{0}\right) .
$$

Here $h$ denotes the step size and $\varphi_{1}$ is the entire function

$$
\varphi_{1}(z)=\frac{\mathrm{e}^{z}-1}{z} \text {. }
$$

Obviously, method (1.6) makes use of the matrix exponential of $A$ and a related function, hence its name 'exponential integrator'.

In the early days of stiff problems, the direct approximation of the matrix exponential and the related $\varphi_{1}$-function was not regarded as practical for large matrices. For this reason, the functions arising were approximated by rational (Padé) approximations which resulted in implicit or semi-implicit Runge-Kutta methods, Rosenbrock methods or W-schemes, just to mention a few well-established methods for stiff problems. The view, however, has changed as new methods for computing or approximating the product of a matrix exponential function with a vector have become available.

Apart from motivating the construction of exponential integrators for various classes of problems, our main intention in this article is to present the mathematics behind exponential integrators. We will derive error bounds that are independent of stiffness or highest frequencies in the system. In order to achieve this aim, we will distinguish - as already mentioned between systems that admit smooth solutions and systems with highly oscillatory solutions. We also emphasize the analytic conditions that underlie the numerical schemes as well as the limitations of the given error bounds. We hope that this will help in choosing the right integrator for a particular application.

In this survey, we will concentrate on the convergence properties of exponential integrators for finite times. We will not discuss further important properties such as long-term behaviour or geometric properties of the discrete flow. For trigonometric integrators, such questions are fully addressed in Hairer, Lubich and Wanner (2006). 
Whenever we speak about the order of convergence in this article, we refer to the so-called stiff order. Recall that a method has stiff order $p$ (for a particular class of problems) if the global error on finite time intervals can be uniformly bounded by $C h^{p}$, where $C$ is a constant that depends on the length of the time interval and on the chosen class of problems, but neither on the stiffness nor on the step size $h$. The stiff order of a method must not be confused with its classical order. The classical order shows up in the non-stiff case for sufficiently small step sizes and is always an upper bound to the stiff order.

For stiff problems with smooth solutions, standard techniques from the theory of stiff differential equations can be used. The local error is determined by inserting the exact solution into the numerical scheme and Taylor-expanding it to determine the defects. Such a Taylor expansion is possible for smooth solutions with bounded derivatives of reasonable size and leads to error bounds in terms of the solution. The linearization $A$ has to fulfil certain conditions to guarantee the stability of the error recursion. A prominent example of this class of problems is that of parabolic partial differential equations, either considered as abstract evolution equations in an appropriate Banach space or their spatial discretizations which result in large systems of stiff ordinary differential equations. For the latter, it is vital to obtain temporal error bounds that are basically independent of the spatial mesh width. In Section 2, we will discuss this approach for various classes of exponential one step and multistep methods.

Problems with highly oscillatory solutions are discussed in Section 3. For these problems, Taylor series expansion of the exact solution is not a viable option. Completely new techniques for constructing efficient methods and for proving error bounds have to be devised. In this section we discuss the construction and error analysis for Magnus integrators for first-order problems, and for trigonometric integrators for second-order problems. In order to avoid resonances, particular filter functions are required. Adiabatic integrators for singularly perturbed problems are briefly discussed as well.

The implementation of exponential integrators often requires the evaluation of the product of a matrix function with a vector. Many different approaches to evaluating this action in an efficient way have been proposed in the literature. In Section 4, we review Chebyshev methods, Krylov subspace methods, interpolation methods based on Leja points, and contour integral methods. For problems with dense matrices, we refer to the review by Higham and Al-Mohy (2010) and to the monograph by Higham (2008). Finally, we give some hints on the mathematical software.

Section 5 is devoted to applications of exponential integrators in science and technology. Exponential integrators, often in combination with splitting methods, have a long tradition in quantum dynamics and chemistry. New applications in mathematical finance and regularization of ill-posed 
problems, just to mention two fields, have emerged recently. We will briefly discuss such applications in this section.

We conclude this review in Section 6 with some comments on how the concept of exponential integrators has developed historically.

Having given this summary, we briefly discuss related topics that are, however, not included in this survey. As already mentioned, many interesting classes of methods for parabolic problems have their origin in the early days of exponential integrators. As the evaluation of the exponential of a large matrix was not regarded as practical at that time, methods were developed using rational approximations instead. Prominent classes are semi-implicit Runge-Kutta methods, Rosenbrock methods and W-methods. These methods will not be discussed further in this survey; we refer to the textbooks by Hairer and Wanner (1996) and Strehmel and Weiner (1992).

Splitting methods are in many respects competitive with exponential integrators; sometimes they are also used in combination with them. We do not discuss splitting methods here, but refer to the survey article of McLachlan and Quispel (2002). Methods for ordinary differential equations on manifolds and Lie group methods will also not be further discussed here. We refer to the review article by Iserles, Munthe-Kaas, Nørsett and Zanna (2000).

\section{Some notation}

We end this Introduction with some words on the notation employed. In order to stress the fundamental difference between smooth and highly oscillatory solutions, we have used different letters to denote them. Smooth solutions in Section 2 are generally denoted by $u$, whereas the letter $\psi$ is reserved for highly oscillatory solutions of first-order equations in Section 3. Finally, $(q, p)$ usually denotes the solution of a second-order problem, rewritten as a first-order system. The first component $q$ is called the position and $p$ is the momentum.

The end of a proof is marked by $\square$, the end of an example by $\diamond$, and the end of an assumption by $\bigcirc$. The conjugate transpose of a matrix $A$ is denoted by $A^{*}$.

Throughout the paper, $C>0$ will denote a generic constant.

\section{Parabolic problems, smooth solutions}

In this section, we consider semilinear problems of the form

$$
u^{\prime}(t)+A u(t)=g(t, u(t)), \quad u\left(t_{0}\right)=u_{0} .
$$

We are most interested in parabolic problems, which can be written in the form (2.1) either as an abstract ordinary differential equation on a suitable function space or as a system of ordinary differential equations in $\mathbb{R}^{n}$ or $\mathbb{C}^{n}$ stemming from a suitable spatial discretization. Throughout the section, 
we will only consider problems with temporally smooth solutions, so that we can always expand the solution in a Taylor series.

After motivating the analytical framework for our error analysis, we will first treat exponential quadrature rules for linear problems. The main part of this section is devoted to exponential Runge-Kutta methods, exponential Rosenbrock methods, exponential multistep methods, exponential general linear methods, and Magnus integrators for semilinear problems (2.1).

\subsection{Preliminaries}

In order to motivate the analytical framework of our error analysis, we start with a simple example.

Example 2.1. We consider the heat equation in one space dimension,

$$
U_{t}(t, x)=U_{x x}(t, x), \quad U(0, x)=U_{0}(x), \quad x \in \Omega=(0, \pi),
$$

subject to homogeneous Dirichlet boundary conditions,

$$
U(t, 0)=U(t, \pi)=0 .
$$

We assume that the initial function satisfies $U_{0} \in L^{2}(\Omega)$. In this case, it is well known that the solution of $(2.2)$ is given by

$$
U(x, t)=\sum_{k=1}^{\infty} \mu_{k} \mathrm{e}^{-k^{2} t} \sin k x,
$$

where the numbers $\mu_{k}$ are the Fourier coefficients of the initial function $U_{0}$,

$$
\mu_{k}=\frac{2}{\pi} \int_{0}^{\pi} U_{0}(x) \sin (k x) \mathrm{d} x .
$$

The assumption $U_{0} \in L^{2}(\Omega)$ is equivalent to $\left(\mu_{k}\right)_{k} \in \ell^{2}$.

For an abstract formulation of (2.2), we consider the linear (differential) operator $A$ defined by

$$
(A v)(x)=-v_{x x}(x) .
$$

Obviously, $A$ is an unbounded operator and not defined for all $v \in L^{2}(\Omega)$. In order to model homogeneous Dirichlet boundary conditions, we consider $A$ on the domain

$$
D(A)=H^{2}(\Omega) \cap H_{0}^{1}(\Omega),
$$

where $H^{2}$ and $H_{0}^{1}$ denote the familiar Sobolev spaces. In one space dimension, functions in $D(A)$ are continuously differentiable and vanish on the boundary of $\Omega$.

This operator has a complete system of orthogonal eigenfunctions $\sin (k x)$ corresponding to the eigenvalues $k^{2}, k=1,2, \ldots$ Due to the isomorphism between $L^{2}$ and $\ell^{2}$, the operator $A$ induces a corresponding operator on $\ell^{2}$. 
An application of $A$ to an $L^{2}$-function corresponds to the multiplication of its Fourier coefficients by $k^{2}$ in $\ell^{2}$. Given a function $\phi: \mathbb{C} \rightarrow \mathbb{C}$, this allows us to define the operator

$$
\phi(t A): L^{2}(\Omega) \rightarrow L^{2}(\Omega), \quad t \geq 0,
$$

given by

$$
\phi(t A) v=\sum_{k=1}^{\infty} \nu_{k} \phi\left(k^{2} t\right) \sin k x \quad \text { for } v(x)=\sum_{k=1}^{\infty} \nu_{k} \sin k x .
$$

Clearly, any function $\phi(\xi)$ bounded for $\xi \geq 0$ defines a bounded operator $\phi(t A)$ for $t \geq 0$. For instance, choosing $\phi(\xi)=\mathrm{e}^{-\xi}$ defines the bounded exponential operator

$$
\mathrm{e}^{-t A}: L^{2}(\Omega) \rightarrow L^{2}(\Omega) .
$$

This operator has the properties of a semigroup, namely $\mathrm{e}^{-0 A}=I$ and

$$
\mathrm{e}^{-t A} \mathrm{e}^{-s A}=\mathrm{e}^{-(t+s) A}, \quad t, s \geq 0 .
$$

Moreover, it satisfies

$$
\left\|\mathrm{e}^{-t A}\right\|_{L^{2}(\Omega) \leftarrow L^{2}(\Omega)} \leq \mathrm{e}^{-t} \leq 1, \quad t \geq 0,
$$

and

$$
\left\|(t A)^{\gamma} \mathrm{e}^{-t A}\right\|_{L^{2}(\Omega) \leftarrow L^{2}(\Omega)}=\sup _{k \geq 1}\left(t k^{2}\right)^{\gamma} \mathrm{e}^{-t k^{2}} \leq C(\gamma), \quad \gamma, t \geq 0 .
$$

Using the above notation, we can now formulate the heat equation as an abstract ordinary differential equation on the Hilbert space $X=L^{2}(\Omega)$ by defining $u(t)$ as the function that maps $x$ to $U(t, x)$ :

$$
u(t)=[x \mapsto U(t, x)] .
$$

Problem (2.2) then reads

$$
u^{\prime}+A u=0, \quad u(0)=u_{0},
$$

and its solution is given by

$$
u(t)=\mathrm{e}^{-t A} u_{0}, \quad t \geq 0,
$$

which looks formally like the familiar matrix exponential in $\mathbb{R}^{n}$.

For homogeneous Neumann boundary conditions, the operator $A$ has to be considered on the domain

$$
D(A)=\left\{v \in H^{2}(\Omega) \mid v_{x}(0)=v_{x}(1)=0\right\} .
$$

This operator has the complete system of orthogonal eigenfunctions $\cos (k x)$, $k=0,1,2, \ldots$, corresponding to the eigenvalues $k^{2}$. Functions of this operator can again be defined with the help of Fourier series. 
The above example motivates the following more general assumption. Background information on semigroups can be found in the textbooks by Henry (1981), Pazy (1992), Lunardi (1995), and Engel and Nagel (2000).

Assumption 2.2. Let $X$ be a Banach space with norm $\|\cdot\|$. We assume that $A$ is a linear operator on $X$ and that $(-A)$ is the infinitesimal generator of a strongly continuous semigroup $\mathrm{e}^{-t A}$ on $X$.

In particular, this assumption implies that there exist constants $C$ and $\omega$ such that

$$
\left\|\mathrm{e}^{-t A}\right\|_{X \leftarrow X} \leq C \mathrm{e}^{\omega t}, \quad t \geq 0 .
$$

Our error analysis will make use of this estimate only.

Example 2.3. Readers unfamiliar with functional analysis may want to think of $X=\mathbb{R}^{n}$ or $X=\mathbb{C}^{n}$. In this case the linear operator can be represented by an $n \times n$ matrix, and $\mathrm{e}^{-t A}$ is just the well-known matrix exponential function. It is important to note that condition (2.6) holds with $\omega=0$ if the field of values of $A$ is contained in the right complex half-plane. For instance, if $A$ is Hermitian positive semidefinite or skew-Hermitian, then $C=1$ and $\omega=0$ hold in the Euclidean norm, independently of the dimension $n$. If $A$ stems from a spatial discretization of a partial differential equation, then using Assumption 2.2 will yield temporal convergence results that are independent of the spatial mesh.

\subsection{Linear problems; exponential quadrature}

In this section, we will derive error bounds for exponential Runge-Kutta discretizations of linear parabolic problems,

$$
u^{\prime}(t)+A u(t)=f(t), \quad u(0)=u_{0},
$$

with a time-invariant operator $A$. The solution of (2.7) at time

$$
t_{n+1}=t_{n}+h_{n}, \quad t_{0}=0, \quad n=0,1, \ldots
$$

is given by the variation-of-constants formula

$$
u\left(t_{n+1}\right)=\mathrm{e}^{-h_{n} A} u\left(t_{n}\right)+\int_{0}^{h_{n}} \mathrm{e}^{-\left(h_{n}-\tau\right) A} f\left(t_{n}+\tau\right) \mathrm{d} \tau .
$$

A first scheme is obtained by approximating the function $f$ within the integral by its interpolation polynomial in certain non-confluent quadrature nodes $c_{1}, \ldots, c_{s}$. This yields the exponential quadrature rule

$$
u_{n+1}=\mathrm{e}^{-h_{n} A} u_{n}+h_{n} \sum_{i=1}^{s} b_{i}\left(-h_{n} A\right) f\left(t_{n}+c_{i} h_{n}\right)
$$


with weights

$$
b_{i}(-h A)=\int_{0}^{1} \mathrm{e}^{-h(1-\theta) A} \ell_{i}(\theta) \mathrm{d} \theta .
$$

Here, $\ell_{i}$ are the familiar Lagrange interpolation polynomials

$$
\ell_{i}(\theta)=\prod_{\substack{m=1 \\ m \neq i}}^{s} \frac{\theta-c_{m}}{c_{i}-c_{m}}, \quad i=1, \ldots, s .
$$

Obviously, the weights $b_{i}(z)$ are linear combinations of the entire functions

$$
\varphi_{k}(z)=\int_{0}^{1} \mathrm{e}^{(1-\theta) z} \frac{\theta^{k-1}}{(k-1) !} \mathrm{d} \theta, \quad k \geq 1 .
$$

These functions satisfy $\varphi_{k}(0)=1 / k$ ! and the recurrence relation

$$
\varphi_{k+1}(z)=\frac{\varphi_{k}(z)-\varphi_{k}(0)}{z}, \quad \varphi_{0}(z)=\mathrm{e}^{z} .
$$

Assumption 2.2 enables us to define the operators

$$
\varphi_{k}(-h A)=\int_{0}^{1} \mathrm{e}^{-h(1-\theta) A} \frac{\theta^{k-1}}{(k-1) !} \mathrm{d} \theta, \quad k \geq 1 .
$$

The following lemma turns out to be crucial.

Lemma 2.4. Under Assumption 2.2, the operators $\varphi_{k}(-h A), k=1,2, \ldots$ are bounded on $X$.

Proof. The boundedness simply follows from the estimate

$$
\left\|\varphi_{k}(-h A)\right\| \leq \int_{0}^{1}\left\|\mathrm{e}^{-h(1-\theta) A}\right\| \frac{\theta^{k-1}}{(k-1) !} \mathrm{d} \theta
$$

and the bound (2.6) on the semigroup.

Example 2.5. For $s=1$ we have

$$
\begin{aligned}
u_{n+1} & =\mathrm{e}^{-h_{n} A} u_{n}+h_{n} \varphi_{1}\left(-h_{n} A\right) f\left(t_{n}+c_{1} h_{n}\right) \\
& =u_{n}+h_{n} \varphi_{1}\left(-h_{n} A\right)\left(f\left(t_{n}+c_{1} h_{n}\right)-A u_{n}\right) .
\end{aligned}
$$

The choice $c_{1}=0$ yields the exponential Euler quadrature rule, while $c_{1}=$ $1 / 2$ corresponds to the exponential midpoint rule.

Example 2.6. For $s=2$ we obtain the weights

$$
\begin{aligned}
& b_{1}(z)=\frac{c_{2}}{c_{2}-c_{1}} \varphi_{1}(z)-\frac{1}{c_{2}-c_{1}} \varphi_{2}(z), \\
& b_{2}(z)=-\frac{c_{1}}{c_{2}-c_{1}} \varphi_{1}(z)+\frac{1}{c_{2}-c_{1}} \varphi_{2}(z) .
\end{aligned}
$$

The choice $c_{1}=0$ and $c_{2}=1$ yields the exponential trapezoidal rule. 
As a generalization to the exponential quadrature rules of collocation type (2.9) considered so far, we will now consider methods where the weights $b_{i}(-h A)$ do not necessarily satisfy condition $(2.9 \mathrm{~b})$. This more general class of methods,

$$
u_{n+1}=\mathrm{e}^{-h_{n} A} u_{n}+h_{n} \sum_{i=1}^{s} b_{i}\left(-h_{n} A\right) f\left(t_{n}+c_{i} h_{n}\right),
$$

only requires the weights $b_{i}(-h A)$ to be uniformly bounded in $h \geq 0$.

In order to analyse (2.12), we expand the right-hand side of (2.8) in a Taylor series with remainder in integral form:

$$
\begin{aligned}
u\left(t_{n+1}\right)= & \mathrm{e}^{-h_{n} A} u\left(t_{n}\right)+\int_{0}^{h_{n}} \mathrm{e}^{-\left(h_{n}-\tau\right) A} f\left(t_{n}+\tau\right) \mathrm{d} \tau \\
= & \mathrm{e}^{-h_{n} A} u\left(t_{n}\right)+h_{n} \sum_{k=1}^{p} \varphi_{k}\left(-h_{n} A\right) h_{n}^{k-1} f^{(k-1)}\left(t_{n}\right) \\
& \quad+\int_{0}^{h_{n}} \mathrm{e}^{-\left(h_{n}-\tau\right) A} \int_{0}^{\tau} \frac{(\tau-\xi)^{p-1}}{(p-1) !} f^{(p)}\left(t_{n}+\xi\right) \mathrm{d} \xi \mathrm{d} \tau .
\end{aligned}
$$

This has to be compared with the Taylor series of the numerical solution (2.12):

$$
\begin{aligned}
u_{n+1}= & \mathrm{e}^{-h_{n} A} u_{n}+h_{n} \sum_{i=1}^{s} b_{i}\left(-h_{n} A\right) f\left(t_{n}+c_{i} h_{n}\right) \\
= & \mathrm{e}^{-h_{n} A} u_{n}+h_{n} \sum_{i=1}^{s} b_{i}\left(-h_{n} A\right) \sum_{k=0}^{p-1} \frac{h_{n}^{k} c_{i}^{k}}{k !} f^{(k)}\left(t_{n}\right) \\
& \quad+h_{n} \sum_{i=1}^{s} b_{i}\left(-h_{n} A\right) \int_{0}^{c_{i} h_{n}} \frac{\left(c_{i} h_{n}-\tau\right)^{p-1}}{(p-1) !} f^{(p)}\left(t_{n}+\tau\right) \mathrm{d} \tau .
\end{aligned}
$$

Obviously the error $e_{n}=u_{n}-u\left(t_{n}\right)$ satisfies

$$
e_{n+1}=\mathrm{e}^{-h_{n} A} e_{n}-\delta_{n+1}
$$

with

$$
\delta_{n+1}=\sum_{j=1}^{p} h_{n}^{j} \psi_{j}\left(-h_{n} A\right) f^{(j-1)}\left(t_{n}\right)+\delta_{n+1}^{(p)},
$$

where

$$
\psi_{j}\left(-h_{n} A\right)=\varphi_{j}\left(-h_{n} A\right)-\sum_{i=1}^{s} b_{i}\left(-h_{n} A\right) \frac{c_{i}^{j-1}}{(j-1) !}
$$


and

$$
\begin{aligned}
\delta_{n+1}^{(p)}=\int_{0}^{h_{n}} \mathrm{e}^{-\left(h_{n}-\tau\right) A} \int_{0}^{\tau} \frac{(\tau-\xi)^{p-1}}{(p-1) !} f^{(p)}\left(t_{n}+\xi\right) \mathrm{d} \xi \mathrm{d} \tau \\
\quad-h_{n} \sum_{i=1}^{s} b_{i}\left(-h_{n} A\right) \int_{0}^{c_{i} h_{n}} \frac{\left(c_{i} h_{n}-\tau\right)^{p-1}}{(p-1) !} f^{(p)}\left(t_{n}+\tau\right) \mathrm{d} \tau .
\end{aligned}
$$

The coefficients (2.17) of the low-order terms in (2.16) being zero turn out to be the desired order conditions of the exponential quadrature rule (2.12).

We are now ready to state our convergence result.

Theorem 2.7. Let Assumption 2.2 be fulfilled and let $f^{(p)} \in L^{1}(0, T)$. For the numerical solution of (2.7), consider the exponential quadrature rule (2.12) with uniformly bounded weights $b_{i}(-h A)$ for $h \geq 0$. If the method satisfies the order conditions

$$
\psi_{j}(-h A)=0, \quad j=1, \ldots, p,
$$

then it converges with order $p$. More precisely, the error bound

$$
\left\|u_{n}-u\left(t_{n}\right)\right\| \leq C \sum_{j=0}^{n-1} h_{j}^{p} \int_{t_{j}}^{t_{j+1}}\left\|f^{(p)}(\tau)\right\| \mathrm{d} \tau
$$

then holds, uniformly on $0 \leq t_{n} \leq T$, with a constant $C$ that depends on $T$, but is independent of the chosen step size sequence.

Proof. Solving the error recursion (2.15) yields the estimate

$$
\left\|e_{n}\right\| \leq \sum_{j=0}^{n-1}\left\|\mathrm{e}^{-\left(t_{n}-t_{j}\right) A}\right\|\left\|\delta_{j}^{(p)}\right\| .
$$

The desired bound follows from the stability bound (2.6) and the assumption on the weights.

Corollary 2.8. Let Assumption 2.2 be fulfilled. Then the exponential quadrature rule (2.9) satisfies the order conditions up to order $s$. It is thus convergent of order $s$.

Proof. The weights $b_{i}$ of the exponential quadrature rule (2.9) satisfy the order conditions (2.18) for $p=s$ by construction. The boundedness of the weights follows from Lemma 2.4.

Theorem 2.7 is not yet optimal for methods whose underlying quadrature rule $\left(b_{i}(0), c_{i}\right)$ satisfies additional order conditions, e.g., $\psi_{s+1}(0)=0$; see Hochbruck and Ostermann (2005b, Section 3) for details. 


\subsection{Exponential Runge-Kutta methods}

For the numerical solution of semilinear problems (2.1), we proceed analogously to the construction of Runge-Kutta methods. We start from the variation-of-constants formula

$$
u\left(t_{n}+h_{n}\right)=\mathrm{e}^{-h_{n} A} u\left(t_{n}\right)+\int_{0}^{h_{n}} \mathrm{e}^{-\left(h_{n}-\tau\right) A} g\left(t_{n}+\tau, u\left(t_{n}+\tau\right)\right) \mathrm{d} \tau .
$$

Since, in contrast to linear problems, the integral now contains the unknown function $u$, we use (2.19) with $h_{n}$ replaced by $c_{i} h_{n}$ to define internal stages. This leads to the following general class of one-step methods:

$$
\begin{aligned}
u_{n+1} & =\chi\left(-h_{n} A\right) u_{n}+h_{n} \sum_{i=1}^{s} b_{i}\left(-h_{n} A\right) G_{n i}, \\
U_{n i} & =\chi_{i}\left(-h_{n} A\right) u_{n}+h_{n} \sum_{j=1}^{s} a_{i j}\left(-h_{n} A\right) G_{n j}, \\
G_{n j} & =g\left(t_{n}+c_{j} h_{n}, U_{n j}\right) .
\end{aligned}
$$

Here, the method coefficients $\chi, \chi_{i}, a_{i j}$, and $b_{i}$ are constructed from exponential functions or (rational) approximations of such functions evaluated at the matrix or operator $\left(-h_{n} A\right)$. For consistency reasons, we always assume that $\chi(0)=\chi_{i}(0)=1$.

It seems worth mentioning that (2.20) reduces to a Runge-Kutta method with coefficients $b_{i}=b_{i}(0)$ and $a_{i j}=a_{i j}(0)$ if we consider the (formal) limit $A \rightarrow 0$. The latter method will henceforth be called the underlying RungeKutta method, while (2.20) will be referred to as an exponential Runge-Kutta method. Throughout this section, we suppose that the underlying RungeKutta method satisfies

$$
\sum_{j=1}^{s} b_{j}(0)=1, \quad \sum_{j=1}^{s} a_{i j}(0)=c_{i}, \quad i=1, \ldots, s,
$$

which makes it invariant under the transformation of (2.1) to autonomous form.

A desirable property of numerical methods is that they preserve equilibria $u^{\star}$ of the autonomous problem

$$
u^{\prime}(t)+A u(t)=g(u(t)) .
$$

Requiring $U_{n i}=u_{n}=u^{\star}$ for all $i$ and $n \geq 0$ immediately yields necessary and sufficient conditions. It turns out that the coefficients of the method have to satisfy

$$
\sum_{j=1}^{s} b_{j}(z)=\frac{\chi(z)-1}{z}, \quad \sum_{j=1}^{s} a_{i j}(z)=\frac{\chi_{i}(z)-1}{z}, \quad i=1, \ldots, s .
$$


Table 2.1. The exponential Runge-Kutta method (2.20) in tableau form. For $\chi$, $\chi_{i}$ being the standard exponential functions (2.22), we omit the last column.

$$
\begin{array}{c|cccc|c}
c_{1} & & & & & \chi_{1}(-h A) \\
c_{2} & a_{21}(-h A) & & & & \chi_{2}(-h A) \\
\vdots & \vdots & \ddots & & \vdots \\
c_{s} & a_{s 1}(-h A) & \ldots & a_{s, s-1}(-h A) & & \chi_{s}(-h A) \\
\hline & b_{1}(-h A) & \ldots & b_{s-1}(-h A) & b_{s}(-h A) & \chi(-h A)
\end{array}
$$

Without further mention, we will assume throughout this section that these conditions are fulfilled. We mainly consider methods with

$$
\chi(z)=\mathrm{e}^{z} \quad \text { and } \quad \chi_{i}(z)=\mathrm{e}^{c_{i} z}, \quad 1 \leq i \leq s .
$$

For this choice, the simplifying assumptions (2.21) read

$$
\sum_{j=1}^{s} b_{j}(z)=\varphi_{1}(z), \quad \sum_{j=1}^{s} a_{i j}(z)=c_{i} \varphi_{1}\left(c_{i} z\right), \quad i=1, \ldots, s .
$$

Our main interest lies in explicit methods for which, due to $c_{1}=0$,

$$
\chi_{1}(z)=1 \quad \text { and } \quad a_{i j}(z)=0, \quad 1 \leq i \leq j \leq s .
$$

The general construction principle together with an error analysis is presented in Hochbruck and Ostermann (2005b). Explicit methods of the form (2.20) have already been proposed by Friedli (1978). He also presented nonstiff order conditions, which, however, are not sufficient to analyse parabolic problems. Methods of the form (2.20) with rational functions $\chi$ and $\chi_{i}$ in place of (2.22) have been proposed and analysed by Strehmel and Weiner (1987, 1992).

As usual, we represent the coefficients of (2.20) in a Butcher tableau: see Table 2.1. With the help of (2.21), the functions $\chi$ and $\chi_{i}$ can be eliminated in (2.20). The numerical scheme then takes the form

$$
\begin{aligned}
u_{n+1} & =u_{n}+h_{n} \sum_{i=1}^{s} b_{i}\left(-h_{n} A\right)\left(G_{n i}-A u_{n}\right), \\
U_{n i} & =u_{n}+h_{n} \sum_{j=1}^{s} a_{i j}\left(-h_{n} A\right)\left(G_{n j}-A u_{n}\right) .
\end{aligned}
$$

Conditions (2.21) also imply that we can restrict ourselves to autonomous problems,

$$
u^{\prime}(t)+A u(t)=g(u(t)), \quad u(0)=u_{0},
$$

since all methods satisfying (2.21) are invariant under the transformation of (2.1) to autonomous form. 
In order to study interesting examples, we have to take care of the norms in which we derive error estimates. Our analysis will make use of the variation-of-constants formula and this will involve bounds of terms of the form

$$
\mathrm{e}^{-t_{n} A}\left(g\left(u\left(t_{n}\right)\right)-g\left(u_{n}\right)\right) \text {. }
$$

Bounding $g$ in the $L^{2}$-norm, for example, would exclude even very simple functions, such as polynomials of degree higher than one. Therefore, we have to refine our framework.

Assumption 2.9. Let $X$ be a Banach space with norm $\|\cdot\|$. We assume that $A$ is a linear operator on $X$ and that $(-A)$ is the infinitesimal generator of an analytic semigroup $\mathrm{e}^{-t A}$ on $X$.

In a similar way to our motivating Example 2.1, generators of analytic semigroups allow us to define fractional powers of the operator. To be more precise, if $A$ satisfies Assumption 2.9, then there exists an $\omega \in \mathbb{R}$ such that $(A+\omega I)^{\alpha}$ is defined for real powers $\alpha$; see Henry (1981) and Pazy (1992). Writing

$$
A u+g(u)=(A+\omega I) u+(g(u)-\omega u)
$$

enables us to set $\omega=0$ without loss of generality. Then Assumption 2.9 implies that there exist constants $C=C(\gamma)$ such that

$$
\left\|\mathrm{e}^{-t A}\right\|_{X \leftarrow X}+\left\|t^{\gamma} A^{\gamma} \mathrm{e}^{-t A}\right\|_{X \leftarrow X} \leq C, \quad \gamma, t \geq 0 .
$$

With this bound at hand, we can estimate the difference of $g$ in a weaker norm:

$$
\left\|\mathrm{e}^{-t_{n} A} A^{\alpha} \cdot A^{-\alpha}\left(g\left(u\left(t_{n}\right)\right)-g\left(u_{n}\right)\right)\right\| \leq C t_{n}^{-\alpha}\left\|A^{-\alpha}\left(g\left(u\left(t_{n}\right)\right)-g\left(u_{n}\right)\right)\right\| .
$$

This observation leads to the following framework, where our main assumption on the nonlinearity $g$ is that of Henry (1981) and Pazy (1992).

Assumption 2.10. For $0 \leq \alpha<1$, let

$$
V=\left\{v \in X \mid A^{\alpha} v \in X\right\}
$$

be a Banach space with norm $\|v\|_{V}=\left\|A^{\alpha} v\right\|$. We assume that $g:[0, T] \times$ $V \rightarrow X$ is locally Lipschitz-continuous in a strip along the exact solution $u$. Thus, there exists a real number $L=L(R, T)$ such that, for all $t \in[0, T]$,

$$
\|g(t, v)-g(t, w)\| \leq L\|v-w\|_{V}
$$

if $\max \left(\|v-u(t)\|_{V},\|w-u(t)\|_{V}\right) \leq R$.

Example 2.11. It is well known that reaction-diffusion equations fit into this abstract framework, as well as the incompressible Navier-Stokes equations in two and three space dimensions; see, e.g., Henry (1981, Chapter 3) and Lunardi (1995, Section 7.3). 
Convergence of the exponential Euler method

We next study the convergence of the simplest method of the class (2.20).

Example 2.12. For $s=1$, the only reasonable choice is the exponential version of Euler's method. Applied to (2.1), it has the form

$$
u_{n+1}=\mathrm{e}^{-h_{n} A} u_{n}+h_{n} \varphi_{1}\left(-h_{n} A\right) g\left(t_{n}, u_{n}\right) .
$$

The Butcher tableau of the method reads

$$
\begin{array}{l|l}
0 & \\
\hline & \varphi_{1}
\end{array} .
$$

In order to simplify the notation, we set

$$
f(t)=g(t, u(t)),
$$

and we consider constant step sizes $h_{n}=h$ only.

Our proofs are heavily based on the representation of the exact solution by the variation-of-constants formula (2.19), which coincides with (2.8) in our notation. We expand $f$ in a Taylor series with remainder in integral form to receive

$$
f\left(t_{n}+\tau\right)=f\left(t_{n}\right)+\int_{0}^{\tau} f^{\prime}\left(t_{n}+\sigma\right) \mathrm{d} \sigma .
$$

Inserting the exact solution into the numerical scheme yields

$$
u\left(t_{n+1}\right)=\mathrm{e}^{-h A} u\left(t_{n}\right)+h \varphi_{1}(-h A) f\left(t_{n}\right)+\delta_{n+1}
$$

where, by (2.13), the defect is given by

$$
\delta_{n+1}=\int_{0}^{h} \mathrm{e}^{-(h-\tau) A} \int_{0}^{\tau} f^{\prime}\left(t_{n}+\sigma\right) \mathrm{d} \sigma \mathrm{d} \tau .
$$

For this defect we have the following estimate.

Lemma 2.13. Let the initial value problem (2.1) satisfy Assumption 2.9. Furthermore, let $0<\beta \leq 1$ and $A^{\beta-1} f^{\prime} \in L^{\infty}(0, T ; V)$. Then

$$
\left\|\sum_{j=0}^{n-1} \mathrm{e}^{-j h A} \delta_{n-j}\right\|_{V} \leq C h \sup _{0 \leq t \leq t_{n}}\left\|A^{\beta-1} f^{\prime}(t)\right\|_{V}
$$

holds with a constant $C$, uniformly in $0 \leq t_{n} \leq T$.

Proof. We denote the supremum by

$$
M:=\sup _{0 \leq t \leq t_{n}}\left\|A^{\beta-1} f^{\prime}(t)\right\|_{V},
$$


and write

$$
\mathrm{e}^{-j h A} \delta_{n-j}=\mathrm{e}^{-j h A} \int_{0}^{h} \mathrm{e}^{-(h-\tau) A} A^{1-\beta} \int_{0}^{\tau} A^{\beta-1} f^{\prime}\left(t_{n-j-1}+\sigma\right) \mathrm{d} \sigma \mathrm{d} \tau .
$$

Using the stability bound (2.27), the term for $j=0$ can be bounded in $V$ by

$$
C M \int_{0}^{h} \tau(h-\tau)^{\beta-1} \mathrm{~d} \tau \leq C M h^{1+\beta},
$$

whereas the remaining sum is bounded by

$$
\left\|\sum_{j=1}^{n-1} \mathrm{e}^{-j h A} \delta_{n-j}\right\|_{V} \leq C M h^{2} \sum_{j=1}^{n-1}(j h)^{\beta-1} \leq C M h \int_{0}^{t_{n-1}} t^{\beta-1} \mathrm{~d} t \leq C M h .
$$

This proves the desired estimate.

For the exponential Euler method, we have the following convergence result.

Theorem 2.14. Let the initial value problem (2.1) satisfy Assumptions 2.9 and 2.10, and consider for its numerical solution the exponential Euler method (2.29). Further assume that $f:[0, T] \rightarrow X$ is differentiable and that $\beta \in(0,1]$ can be chosen such that $A^{\beta-1} f^{\prime} \in L^{\infty}(0, T ; V)$. Then, the error bound

$$
\left\|u_{n}-u\left(t_{n}\right)\right\|_{V} \leq C h \sup _{0 \leq t \leq t_{n}}\left\|A^{\beta-1} f^{\prime}(t)\right\|_{V}
$$

holds uniformly in $0 \leq n h \leq T$. The constant $C$ depends on $T$, but it is independent of $n$ and $h$.

Proof. The exponential Euler method satisfies the error recursion

$$
e_{n+1}=\mathrm{e}^{-h A} e_{n}+h \varphi_{1}(-h A)\left(g\left(t_{n}, u_{n}\right)-f\left(t_{n}\right)\right)-\delta_{n+1}
$$

with defect $\delta_{n+1}$ defined in (2.32). Solving this recursion yields

$$
e_{n}=h \sum_{j=0}^{n-1} \mathrm{e}^{-(n-j-1) h A} \varphi_{1}(-h A)\left(g\left(t_{j}, u_{j}\right)-f\left(t_{j}\right)\right)-\sum_{j=0}^{n-1} \mathrm{e}^{-j h A} \delta_{n-j} .
$$

Using (2.27), Assumption 2.10 and Lemma 2.13, we may estimate this in $V$ by

$$
\left\|e_{n}\right\|_{V} \leq C h \sum_{j=0}^{n-2} t_{n-j-1}^{-\alpha}\left\|e_{j}\right\|_{V}+C h^{1-\alpha}\left\|e_{n-1}\right\|_{V}+C h \sup _{0 \leq t \leq t_{n}}\left\|A^{\beta-1} f^{\prime}(t)\right\|_{V} .
$$

The application of Lemma 2.15 concludes the proof.

In the previous proof we used the following standard Discrete Gronwall Lemma. For its proof see Dixon and McKee (1986), for instance. 
Lemma 2.15. (Discrete Gronwall Lemma) For $h>0$ and $T>0$, let $0 \leq t_{n}=n h \leq T$. Further assume that the sequence of non-negative numbers $\varepsilon_{n}$ satisfies the inequality

$$
\varepsilon_{n} \leq a h \sum_{\nu=1}^{n-1} t_{n-\nu}^{-\rho} \varepsilon_{\nu}+b t_{n}^{-\sigma}
$$

for $0 \leq \rho, \sigma<1$ and $a, b \geq 0$. Then the estimate

$$
\varepsilon_{n} \leq C b t_{n}^{-\sigma}
$$

holds, where the constant $C$ depends on $\rho, \sigma, a$, and on $T$.

Convergence of the higher-order exponential Runge-Kutta methods

The convergence analysis of higher-order methods turns out to be much more complicated than that for the exponential Euler scheme, due to the low order of the internal stages. Moreover, it requires additional assumptions on the nonlinearity $g$.

Assumption 2.16. We assume that (2.1) possesses a sufficiently smooth solution $u:[0, T] \rightarrow V$ with derivatives in $V$, and that $g:[0, T] \times V \rightarrow X$ is sufficiently often Fréchet-differentiable in a strip along the exact solution. All occurring derivatives are assumed to be uniformly bounded.

Note that, under the above assumption, the composition

$$
f:[0, T] \rightarrow X: t \mapsto f(t)=g(t, u(t))
$$

is a smooth mapping, too. This will be used frequently.

As usual for the analysis of stiff problems with smooth solutions, and as we have seen for the exponential Euler method, we start by inserting the exact solution into the numerical scheme. This yields

$$
\begin{gathered}
u\left(t_{n}+c_{i} h\right)=\mathrm{e}^{-c_{i} h A} u\left(t_{n}\right)+h \sum_{j=1}^{i-1} a_{i j}(-h A) f\left(t_{n}+c_{j} h\right)+\Delta_{n i}, \\
u\left(t_{n+1}\right)=\mathrm{e}^{-h A} u\left(t_{n}\right)+h \sum_{i=1}^{s} b_{i}(-h A) f\left(t_{n}+c_{i} h\right)+\delta_{n+1},
\end{gathered}
$$

with defect $\delta_{n+1}$ given in (2.16), and

$$
\Delta_{n i}=\sum_{j=1}^{q_{i}} h^{j} \psi_{j, i}(-h A) f^{(j-1)}\left(t_{n}\right)+\Delta_{n i}^{\left(q_{i}\right)},
$$

where

$$
\psi_{j, i}(-h A)=\varphi_{j}\left(-c_{i} h A\right) c_{i}^{j}-\sum_{k=1}^{i-1} a_{i k}(-h A) \frac{c_{k}^{j-1}}{(j-1) !}
$$


and

$$
\begin{aligned}
\Delta_{n i}^{\left(q_{i}\right)}= & \int_{0}^{c_{i} h} \mathrm{e}^{-\left(c_{i} h-\tau\right) A} \int_{0}^{\tau} \frac{(\tau-\sigma)^{q_{i}-1}}{\left(q_{i}-1\right) !} f^{\left(q_{i}\right)}\left(t_{n}+\sigma\right) \mathrm{d} \sigma \mathrm{d} \tau \\
& -h \sum_{k=1}^{i-1} a_{i k}(-h A) \int_{0}^{c_{k} h} \frac{\left(c_{k} h-\sigma\right)^{q_{i}-1}}{\left(q_{i}-1\right) !} f^{\left(q_{i}\right)}\left(t_{n}+\sigma\right) \mathrm{d} \sigma
\end{aligned}
$$

Note that $\psi_{1, i}=0$ due to the simplifying assumptions (2.23), but - as for all explicit Runge-Kutta schemes - it is not possible to achieve $\psi_{2, i}=0$ for all $i$. This implies that the internal stages are of order one only, which makes the construction of higher-order methods quite involved. We refer to Hochbruck and Ostermann (2005a) for details, and only state the main result here.

Theorem 2.17. Let the initial value problem (2.1) satisfy Assumptions 2.9 and 2.16 with $V=X$ and consider for its numerical solution an explicit exponential Runge-Kutta method (2.20) satisfying (2.22)-(2.24). For $2 \leq$ $p \leq 4$, assume that the order conditions of Table 2.2 hold up to order $p-1$ and that $\psi_{p}(0)=0$. Further assume that the remaining conditions of order $p$ hold in a weaker form with $b_{i}(0)$ instead of $b_{i}(-h A)$ for $2 \leq i \leq s$. Then the numerical solution $u_{n}$ satisfies the error bound

$$
\left\|u_{n}-u\left(t_{n}\right)\right\| \leq C h^{p}
$$

uniformly in $0 \leq n h \leq T$. The constant $C$ depends on $T$, but it is independent of $n$ and $h$.

We now consider some particular examples. We start with second-order methods.

Example 2.18. Second-order methods require two internal stages at least. For two stages, the order conditions taken from Table 2.2 are

$$
\begin{aligned}
b_{1}(-h A)+b_{2}(-h A) & =\varphi_{1}(-h A), \\
b_{2}(-h A) c_{2} & =\varphi_{2}(-h A), \\
a_{21}(-h A) & =c_{2} \varphi_{1}\left(-c_{2} h A\right) .
\end{aligned}
$$

A straightforward elimination leads to the following one-parameter family of exponential Runge-Kutta methods:

$$
\begin{array}{c|cc}
0 & & \\
c_{2} & c_{2} \varphi_{1,2} & \\
\hline & \varphi_{1}-\frac{1}{c_{2}} \varphi_{2} & \frac{1}{c_{2}} \varphi_{2}
\end{array} .
$$

Here and in the following Butcher tableaux, we use the short notation

$$
\varphi_{j, k}=\varphi_{j}\left(-c_{k} h A\right), \quad \varphi_{j}=\varphi_{j}(-h A) .
$$


Table 2.2. Stiff order conditions for explicit exponential Runge-Kutta methods for $\alpha=0$. Here $J$ and $K$ denote arbitrary bounded operators on $X$. The functions $\psi_{i}$ and $\psi_{k, \ell}$ are defined in (2.17) and (2.37), respectively.

\begin{tabular}{ccl}
\hline \hline Number & Order & Order condition \\
\hline 1 & 1 & $\psi_{1}(-h A)=0$ \\
\hline 2 & 2 & $\psi_{2}(-h A)=0$ \\
3 & 2 & $\psi_{1, i}(-h A)=0$ \\
\hline 4 & 3 & $\psi_{3}(-h A)=0$ \\
5 & 3 & $\sum_{i=1}^{s} b_{i}(-h A) J \psi_{2, i}(-h A)=0$ \\
\hline 6 & 4 & $\psi_{4}(-h A)=0$ \\
7 & 4 & $\sum_{i=1}^{s} b_{i}(-h A) J \psi_{3, i}(-h A)=0$ \\
8 & 4 & $\sum_{i=1}^{s} b_{i}(-h A) J \sum_{j=2}^{i-1} a_{i j}(-h A) J \psi_{2, j}(-h A)=0$ \\
9 & 4 & $\sum_{i=1}^{s} b_{i}(-h A) c_{i} K \psi_{2, i}(-h A)=0$ \\
\hline
\end{tabular}

It is also possible to omit the function $\varphi_{2}$ by weakening condition $(2.38 \mathrm{~b})$ to $b_{2}(0) c_{2}=\varphi_{2}(0)=\frac{1}{2}$. This yields another one-parameter family of methods:

$$
\begin{array}{c|cc}
0 & & \\
c_{2} & c_{2} \varphi_{1,2} & \\
\hline & \left(1-\frac{1}{2 c_{2}}\right) \varphi_{1} & \frac{1}{2 c_{2}} \varphi_{1}
\end{array} .
$$

The most attractive choice here is $c_{2}=\frac{1}{2}$, which yields $b_{1}=0$.

Methods (2.39) and (2.40) have already been proposed by Strehmel and Weiner (1992, Example 4.2.2) in the context of adaptive Runge-Kutta methods, where the functions $\varphi_{j}$ are usually approximated by certain rational functions. It is shown in Strehmel and Weiner (1992, Section 4.5.3) that both methods are B-consistent of order one.

The construction of various families of third-order methods can be found in Hochbruck and Ostermann (2005a). In addition, a discussion of the convergence of some related three-stage methods which can be found in the literature is presented there. Among these are the three-stage adaptive Runge-Kutta method of Strehmel and Weiner (1992, Example 4.5.4), the ETD3RK scheme by Cox and Matthews (2002) and the ETD2CF3 method, 
which is a variant of the commutator-free Lie group method CF3 of Celledoni, Marthinsen and Owren (2003).

We next discuss in more detail some four-stage methods that have recently been discussed in the literature.

Example 2.19. Cox and Matthews (2002) proposed the following exponential variant of the classical Runge-Kutta method:

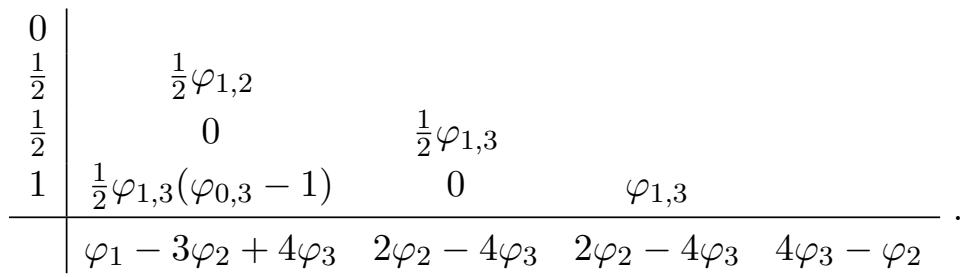

This method satisfies conditions $1-4$ of Table 2.2, the weakened but sufficient condition $6\left(\psi_{4}(0)=0\right)$, but not conditions 5, 7, 8 and 9. However, it satisfies a weakened form of conditions 5 and 9 (because $\psi_{2,2}(0)+\psi_{2,3}(0)=0$ and $\psi_{2,4}(0)=0$ ), and a very weak form of conditions 7 and 8 (where all arguments are evaluated for $A=0$ ). In the worst case, this leads to an order reduction to order two only.

The method by Krogstad (2005) is given by

$$
\begin{array}{c|cccc}
0 & & & & \\
\frac{1}{2} & \frac{1}{2} \varphi_{1,2} & & & \\
\frac{1}{2} & \frac{1}{2} \varphi_{1,3}-\varphi_{2,3} & \varphi_{2,3} & & \\
1 & \varphi_{1,4}-2 \varphi_{2,4} & 0 & 2 \varphi_{2,4} & \\
\hline & \varphi_{1}-3 \varphi_{2}+4 \varphi_{3} & 2 \varphi_{2}-4 \varphi_{3} & 2 \varphi_{2}-4 \varphi_{3} & -\varphi_{2}+4 \varphi_{3}
\end{array} .
$$

This method satisfies conditions $1-5$ and 9 of Table 2.2 , the weakened but sufficient condition $6\left(\psi_{4}(0)=0\right)$, but not conditions 7 and 8 , which are only satisfied in a very weak form (where all arguments are evaluated for $A=0$ ). In the worst case, this leads to an order reduction to order three.

Strehmel and Weiner (1992, Example 4.5.5) suggested the scheme

$$
\begin{array}{c|cccc}
0 & & & & \\
\frac{1}{2} & \frac{1}{2} \varphi_{1,2} & & & \\
\frac{1}{2} & \frac{1}{2} \varphi_{1,3}-\frac{1}{2} \varphi_{2,3} & \frac{1}{2} \varphi_{2,3} & & \\
1 & \varphi_{1,4}-2 \varphi_{2,4} & -2 \varphi_{2,4} & 4 \varphi_{2,4} & \\
\hline & \varphi_{1}-3 \varphi_{2}+4 \varphi_{3} & 0 & 4 \varphi_{2}-8 \varphi_{3} & -\varphi_{2}+4 \varphi_{3}
\end{array} .
$$

This method satisfies the conditions of Table 2.2 in exactly the same way as Krogstad's method. It thus converges in our situation, with order three in the worst case. Strehmel and Weiner (1992) proved that the method is B-consistent of order two. 
The question whether it is possible to modify the coefficients of the above four-stage methods in such a way that they have order four for semilinear parabolic problems was answered in the negative by Hochbruck and Ostermann $(2005 a)$. There, the following five-stage method was constructed:

$$
\begin{array}{c|ccccc}
0 & & & & & \\
\frac{1}{2} & \frac{1}{2} \varphi_{1,2} & & & & \\
\frac{1}{2} & \frac{1}{2} \varphi_{1,3}-\varphi_{2,3} & \varphi_{2,3} & & & \\
1 & \varphi_{1,4}-2 \varphi_{2,4} & \varphi_{2,4} & \varphi_{2,4} & & \\
\frac{1}{2} & \frac{1}{2} \varphi_{1,5}-2 a_{5,2}-a_{5,4} & a_{5,2} & a_{5,2} & \frac{1}{4} \varphi_{2,5}-a_{5,2} & \\
\hline & \varphi_{1}-3 \varphi_{2}+4 \varphi_{3} & 0 & 0 & -\varphi_{2}+4 \varphi_{3} & 4 \varphi_{2}-8 \varphi_{3}
\end{array}
$$

with

$$
a_{5,2}=\frac{1}{2} \varphi_{2,5}-\varphi_{3,4}+\frac{1}{4} \varphi_{2,4}-\frac{1}{2} \varphi_{3,5} .
$$

Under the assumptions of Theorem 2.17, this method has order four.

\subsection{Exponential Rosenbrock methods}

We now turn to the time discretization of (possibly abstract) differential equations in autonomous form,

$$
u^{\prime}(t)=F(u(t)), \quad u\left(t_{0}\right)=u_{0} .
$$

The numerical schemes considered are based on a continuous linearization of (2.44) along the numerical solution. For a given point $u_{n}$ in the state space, this linearization is

$$
\begin{gathered}
u^{\prime}(t)=J_{n} u(t)+g_{n}(u(t)), \\
J_{n}=\mathrm{D} F\left(u_{n}\right)=\frac{\partial F}{\partial u}\left(u_{n}\right), \quad g_{n}(u(t))=F(u(t))-J_{n} u(t),
\end{gathered}
$$

with $J_{n}$ denoting the Jacobian of $F$ and $g_{n}$ the nonlinear remainder evaluated at $u_{n}$, respectively. The numerical schemes will make explicit use of these quantities.

Let $u_{n}$ denote the numerical approximation to the solution of (2.44) at time $t_{n}$. Its value at $t_{0}$ is given by the initial condition. Applying an explicit exponential Runge-Kutta scheme (2.35) to (2.45), we obtain the following class of exponential Rosenbrock methods:

$$
\begin{aligned}
U_{n i} & =\mathrm{e}^{c_{i} h_{n} J_{n}} u_{n}+h_{n} \sum_{j=1}^{i-1} a_{i j}\left(h_{n} J_{n}\right) g_{n}\left(U_{n j}\right), \quad 1 \leq i \leq s, \\
u_{n+1} & =\mathrm{e}^{h_{n} J_{n}} u_{n}+h_{n} \sum_{i=1}^{s} b_{i}\left(h_{n} J_{n}\right) g_{n}\left(U_{n i}\right) .
\end{aligned}
$$


Methods of this form were proposed by Hochbruck and Ostermann (2006). Without further mention, we will assume that the methods fulfil the simplifying assumptions (2.23). Therefore, $c_{1}=0$ and consequently $U_{n 1}=u_{n}$. Methods that satisfy the simplifying assumptions possess several interesting features. In particular, they preserve equilibria of (2.44), they have small defects, which in turn leads to simple order conditions for stiff problems (see Table 2.3 below), they allow a reformulation for efficient implementation, and they can easily be extended to non-autonomous problems.

Example 2.20. The well-known exponential Rosenbrock-Euler method is given by

$$
\begin{aligned}
u_{n+1} & =\mathrm{e}^{h_{n} J_{n}} u_{n}+h_{n} \varphi_{1}\left(h_{n} J_{n}\right) g_{n}\left(u_{n}\right) \\
& =u_{n}+h_{n} \varphi_{1}\left(h_{n} J_{n}\right) F\left(u_{n}\right) .
\end{aligned}
$$

It is computationally attractive since it requires only one matrix function per step.

The first exponential integrator based on the local linearization (2.45) was proposed by Pope (1963). Tokman (2006) pursued the same approach leading to her EPI time integration. In her paper, multistep methods were also constructed.

Related approaches include the local linearization schemes by Ramos and García-López (1997) and De la Cruz, Biscay, Carbonell, Ozaki and Jimenez (2007). Implicit methods combined with fixed-point iteration and Krylov subspace approximations have been proposed by Friesner, Tuckerman, Dornblaser and Russo (1989).

For the implementation of an exponential Rosenbrock method it is crucial to approximate the application of matrix functions to vectors efficiently. We therefore suggest expressing the vectors $g_{n}\left(U_{n j}\right)$ as

$$
g_{n}\left(U_{n j}\right)=g_{n}\left(u_{n}\right)+D_{n j}, \quad 2 \leq j \leq s .
$$

A similar idea was used in Tokman (2006). Due to the simplifying assumptions (2.23), the method (2.46) takes the equivalent form

$$
\begin{aligned}
U_{n i} & =u_{n}+c_{i} h_{n} \varphi_{1}\left(c_{i} h_{n} J_{n}\right) F\left(u_{n}\right)+h_{n} \sum_{j=2}^{i-1} a_{i j}\left(h_{n} J_{n}\right) D_{n j}, \\
u_{n+1} & =u_{n}+h_{n} \varphi_{1}\left(h_{n} J_{n}\right) F\left(u_{n}\right)+h_{n} \sum_{i=2}^{s} b_{i}\left(h_{n} J_{n}\right) D_{n i} .
\end{aligned}
$$

Hence, each stage of the method consists of a perturbed exponential Rosenbrock-Euler step (2.47).

The main motivation for this reformulation is that the vectors $D_{n i}$ are expected to be small in norm. When computing the application of matrix functions to these vectors with some Krylov subspace method, this should 
be possible in a low-dimensional subspace. Consequently, only one computationally expensive Krylov approximation will be required in each time step, namely that involving $F\left(u_{n}\right)$. A similar idea has also been used in Hochbruck, Lubich and Selhofer (1998) to make the code exp4 efficient.

The proposed method can easily be extended to non-autonomous problems,

$$
u^{\prime}=F(t, u), \quad u\left(t_{0}\right)=u_{0},
$$

by rewriting the problem in autonomous form,

$$
U^{\prime}=\mathcal{F}(U), \quad U=\left[\begin{array}{l}
t \\
u
\end{array}\right], \quad \mathcal{F}(U)=\left[\begin{array}{c}
1 \\
F(t, u)
\end{array}\right],
$$

with Jacobian

$$
\mathcal{J}_{n}=\left[\begin{array}{cc}
0 & 0 \\
v_{n} & J_{n}
\end{array}\right], \quad v_{n}=\frac{\partial F}{\partial t}\left(t_{n}, u_{n}\right), \quad J_{n}=\frac{\partial F}{\partial u}\left(t_{n}, u_{n}\right) .
$$

This transformation is standard for Rosenbrock methods as well (see Hairer and Wanner (1996)), but it changes a linear non-autonomous problem into a nonlinear one.

In order to apply our method to the autonomous system (2.50), we have to compute matrix functions of $\mathcal{J}_{n}$. Using Cauchy's integral formula and exploiting the special structure of $\mathcal{J}_{n}$, we get

$$
\varphi_{k}\left(h_{n} \mathcal{J}_{n}\right)=\left[\begin{array}{cc}
\varphi_{k}(0) & 0 \\
h_{n} \varphi_{k+1}\left(h_{n} J_{n}\right) v_{n} & \varphi_{k}\left(h_{n} J_{n}\right)
\end{array}\right] .
$$

In our formulation, we will again work with the smaller quantities

$$
D_{n j}=g_{n}\left(t_{n}+c_{j} h_{n}, U_{n j}\right)-g_{n}\left(t_{n}, u_{n}\right)
$$

where

$$
g_{n}(t, u)=F(t, u)-J_{n} u-v_{n} t .
$$

Applying method (2.48) to the autonomous formulation (2.50), we get

$$
\begin{aligned}
U_{n i}=u_{n} & +h_{n} c_{i} \varphi_{1}\left(c_{i} h_{n} J_{n}\right) F\left(t_{n}, u_{n}\right) \\
& +h_{n}^{2} c_{i}^{2} \varphi_{2}\left(c_{i} h_{n} J_{n}\right) v_{n}+h_{n} \sum_{j=2}^{i-1} a_{i j}\left(h_{n} J_{n}\right) D_{n j}, \\
u_{n+1}=u_{n} & +h_{n} \varphi_{1}\left(h_{n} J_{n}\right) F\left(t_{n}, u_{n}\right) \\
& +h_{n}^{2} \varphi_{2}\left(h_{n} J_{n}\right) v_{n}+h_{n} \sum_{i=2}^{s} b_{i}\left(h_{n} J_{n}\right) D_{n i} .
\end{aligned}
$$

This is the format of an exponential Rosenbrock method for non-autonomous problems (2.49). 
Example 2.21. The exponential Rosenbrock-Euler method for non-autonomous problems is given by

$$
u_{n+1}=u_{n}+h_{n} \varphi_{1}\left(h_{n} J_{n}\right) F\left(u_{n}\right)+h_{n}^{2} \varphi_{2}\left(h_{n} J_{n}\right) v_{n},
$$

with $J_{n}$ and $v_{n}$ defined in $(2.50 \mathrm{~b})$. This scheme was proposed by Pope (1963).

In the subsequent analysis, we restrict our attention to autonomous semilinear problems,

$$
u^{\prime}(t)=F(u(t)), \quad F(u)=-A u+g(u), \quad u\left(t_{0}\right)=u_{0} .
$$

This implies that $(2.45 \mathrm{~b})$ takes the form

$$
J_{n}=-A+\frac{\partial g}{\partial u}\left(u_{n}\right), \quad g_{n}(u(t))=g(u(t))-\frac{\partial g}{\partial u}\left(u_{n}\right) u(t) .
$$

We suppose that $A$ satisfies Assumption 2.2. Our main hypothesis on the nonlinearity $g$ is Assumption 2.16 with $V=X$. The latter assumption implies that the Jacobian

$$
J=J(u)=\mathrm{D} F(u)=\frac{\partial F}{\partial u}(u)
$$

satisfies the Lipschitz condition

$$
\|J(u)-J(v)\|_{X \leftarrow X} \leq C\|u-v\|
$$

in a neighbourhood of the exact solution.

Convergence of higher-order exponential Rosenbrock methods

Theorem 2.22. Suppose the initial value problem (2.54) satisfies Assumptions 2.2 and 2.16 with $V=X$. Consider for its numerical solution an explicit exponential Rosenbrock method (2.46) that fulfils the order conditions of Table 2.3 up to order $p$ for some $2 \leq p \leq 4$. Further, let the step size sequence $h_{j}$ satisfy the condition

$$
\sum_{k=1}^{n-1} \sum_{j=0}^{k-1} h_{j}^{p+1} \leq C_{\mathrm{H}}
$$

with a constant $C_{\mathrm{H}}$ that is uniform in $t_{0} \leq t_{n} \leq T$. Then, for $C_{\mathrm{H}}$ sufficiently small, the numerical method converges with order $p$. In particular, the numerical solution satisfies the error bound

$$
\left\|u_{n}-u\left(t_{n}\right)\right\| \leq C \sum_{j=0}^{n-1} h_{j}^{p+1}
$$

uniformly on $t_{0} \leq t_{n} \leq T$. The constant $C$ is independent of the chosen step size sequence satisfying (2.57). 
Table 2.3. Stiff order conditions for exponential

Rosenbrock methods applied to autonomous problems.

\begin{tabular}{clc}
\hline \hline Number & Order condition & Order \\
\hline 1 & $\sum_{i=1}^{s} b_{i}(z)=\varphi_{1}(z)$ & 1 \\
2 & $\sum_{j=1}^{i-1} a_{i j}(z)=c_{i} \varphi_{1}\left(c_{i} z\right), \quad 2 \leq i \leq s$ & 2 \\
3 & $\sum_{i=2}^{s} b_{i}(z) c_{i}^{2}=2 \varphi_{3}(z)$ & 3 \\
4 & $\sum_{i=2}^{s} b_{i}(z) c_{i}^{3}=6 \varphi_{4}(z)$ & 4 \\
\hline
\end{tabular}

Proof. We present the proof for the exponential Rosenbrock-Euler method only. The proof for higher-order methods can be found in Hochbruck, Ostermann and Schweitzer $(2009 c)$.

To simplify the notation, we write $f_{n}(t)=g_{n}(u(t))$. Inserting the exact solution into the numerical scheme yields

$$
u\left(t_{n+1}\right)=\mathrm{e}^{h_{n} J_{n}} u\left(t_{n}\right)+h_{n} \varphi_{1}\left(h_{n} J_{n}\right) f_{n}\left(t_{n}\right)+\delta_{n+1}
$$

with defect $\delta_{n+1}$. Next, by using the variation-of-constants formula (2.8), we obtain

$$
u\left(t_{n+1}\right)=\mathrm{e}^{h_{n} J_{n}} u\left(t_{n}\right)+\int_{0}^{h_{n}} \mathrm{e}^{\left(h_{n}-\tau\right) J_{n}} f_{n}\left(t_{n}+\tau\right) \mathrm{d} \tau .
$$

Taylor expansion of $f$ within the integral yields

$$
f_{n}\left(t_{n}+\tau\right)=f_{n}\left(t_{n}\right)+\tau f_{n}^{\prime}\left(t_{n}\right)+\int_{0}^{\tau}(\tau-\xi) f_{n}^{\prime \prime}\left(t_{n}+\xi\right) \mathrm{d} \xi
$$

where, by definition of $g_{n}$,

$$
f_{n}^{\prime}\left(t_{n}\right)=\left(\frac{\partial g}{\partial u}\left(u\left(t_{n}\right)\right)-\frac{\partial g}{\partial u}\left(u_{n}\right)\right) u^{\prime}\left(t_{n}\right)
$$

We thus have

$$
\delta_{n+1}=h_{n}^{2} \varphi_{2}\left(h_{n} J_{n}\right) f_{n}^{\prime}\left(t_{n}\right)+\int_{0}^{h_{n}} \mathrm{e}^{\left(h_{n}-\tau\right) J_{n}} \int_{0}^{\tau}(\tau-\xi) f_{n}^{\prime \prime}\left(t_{n}+\xi\right) \mathrm{d} \xi \mathrm{d} \tau,
$$

and this gives the bound

$$
\left\|\delta_{n+1}\right\| \leq C\left(h_{n}^{2}\left\|e_{n}\right\|+h_{n}^{3}\right) .
$$

Subtracting (2.59) from the numerical solution leads to the error recursion

$$
e_{n+1}=\mathrm{e}^{h_{n} J_{n}} e_{n}+h_{n} \varrho_{n}-\delta_{n+1}, \quad e_{0}=0,
$$

with

$$
\varrho_{n}=\varphi_{1}\left(h_{n} J_{n}\right)\left(g_{n}\left(u_{n}\right)-f_{n}\left(t_{n}\right)\right) .
$$


Using the Lipschitz condition (2.56) in

$$
g_{n}\left(u_{n}\right)-f_{n}\left(t_{n}\right)=\int_{0}^{1}\left(\frac{\partial g_{n}}{\partial u}\left(u\left(t_{n}\right)+\theta e_{n}\right)-\frac{\partial g_{n}}{\partial u}\left(u_{n}\right)\right) e_{n} \mathrm{~d} \theta,
$$

we obtain

$$
\left\|g_{n}\left(u_{n}\right)-f_{n}\left(t_{n}\right)\right\| \leq C\left\|e_{n}\right\|^{2} .
$$

Solving the error recursion (2.62) and using $e_{0}=0$, we obtain

$$
e_{n}=\sum_{j=0}^{n-1} h_{j} \mathrm{e}^{h_{n-1} J_{n-1}} \cdots \mathrm{e}^{h_{j+1} J_{j+1}}\left(\varrho_{j}-h_{j}^{-1} \delta_{j+1}\right) .
$$

Employing (2.61) and (2.63), we obtain the bound

$$
\left\|\varrho_{j}\right\|+h_{j}^{-1}\left\|\delta_{j+1}\right\| \leq C\left(h_{j}\left\|e_{j}\right\|+\left\|e_{j}\right\|^{2}+h_{j}^{2}\right) .
$$

Inserting this into (2.64) and using the stability estimate of Hochbruck et al. (2009c, Theorem 3.7), we have

$$
\left\|e_{n}\right\| \leq C \sum_{j=0}^{n-1} h_{j}\left(\left\|e_{j}\right\|^{2}+h_{j}\left\|e_{j}\right\|+h_{j}^{2}\right) .
$$

The constant in this estimate is uniform as long as

$$
\sum_{j=1}^{n-1}\left\|e_{j}\right\| \leq C_{\mathrm{A}}
$$

holds uniformly on $t_{0} \leq t_{n} \leq T$. The application of a version of the Discrete Gronwall Lemma (Lemma 2.15) for variable step sizes (see, e.g., Emmrich (2005)) to (2.66) then shows the desired bound (2.58).

It still remains to verify that condition (2.67) holds with a uniform bound $C_{\mathrm{A}}$. This now follows recursively from (2.58) and our assumption on the step size sequence (2.57) with $C_{\mathrm{H}}$ sufficiently small.

The well-known exponential Rosenbrock-Euler method (2.47) obviously satisfies condition 1 of Table 2.3, while condition 2 is void. Therefore, it is second-order convergent for problems satisfying our analytic framework. A possible error estimator for (2.47) is described in Caliari and Ostermann (2009).

From the order conditions of Table 2.3, we constructed pairs of embedded methods of order three and four in Hochbruck et al. (2009c). For our variable step size implementation, we consider (2.46b) together with an embedded approximation

$$
\widehat{u}_{n+1}=\mathrm{e}^{h_{n} J_{n}} u_{n}+h_{n} \sum_{i=1}^{s} \widehat{b}_{i}\left(h_{n} J_{n}\right) g_{n}\left(U_{n i}\right),
$$

which relies on the same stages $U_{n i}$. 
Example 2.23. The scheme exprb32 consists of a third-order exponential Rosenbrock method with a second-order error estimator (the exponential Rosenbrock-Euler method). Its coefficients are

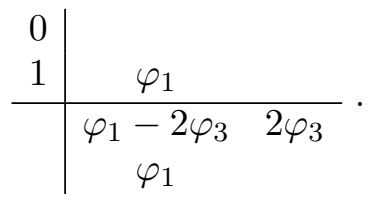

The additional bottom line in the Butcher tableau contains the weight $\widehat{b}_{1}$ of the embedded method (2.68).

Example 2.24. The scheme exprb43 is a fourth-order method with a third-order error estimator. Its coefficients are

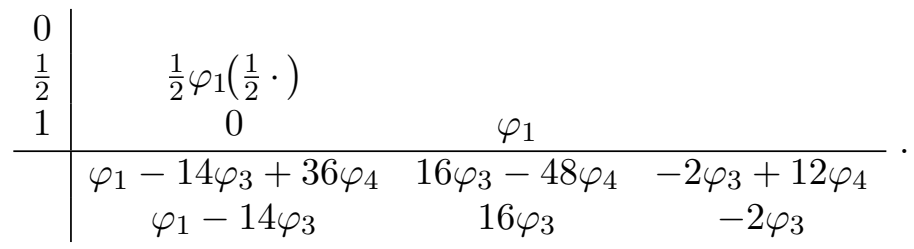

Note that the internal stages of the above methods are just exponential Rosenbrock-Euler steps. This leads to simple methods that can be implemented cheaply.

Evidently, the order conditions of Table 2.3 imply that the weights of any third-order method have to depend on $\varphi_{3}$, whereas those of any fourth-order method depend on $\varphi_{3}$ and $\varphi_{4}$ (in addition to $\varphi_{1}$ ).

Example 2.25. Hochbruck et al. (1998) proposed the following class of exponential integrators, which uses the $\varphi_{1}$-function only:

$$
\begin{aligned}
k_{i} & =\varphi_{1}\left(\gamma h J_{n}\right)\left(J_{n} u_{n}+g_{n}\left(U_{n i}\right)+h J_{n} \sum_{j=1}^{i-1} \beta_{i j} k_{j}\right), \quad i=1, \ldots, s, \\
U_{n i} & =u_{n}+h \sum_{j=1}^{i-1} \alpha_{i j} k_{j}, \\
u_{n+1} & =u_{n}+h \sum_{i=1}^{s} b_{i} k_{i} .
\end{aligned}
$$

Here, $\gamma, \alpha_{i j}, \beta_{i j}, b_{i}$, are scalar coefficients that determine the method. This method is of the form $(2.20)$, with $\chi_{i}, i=1, \ldots, s$ being linear combinations of exponential functions and with $\chi(z)=\mathrm{e}^{z}$. The method exp4 proposed by Hochbruck et al. (1998) can be interpreted as a three-stage exponential Rosenbrock-type method, which is of classical order four. However, for an efficient implementation, it should be written as a seven-stage method, 
which uses only three function evaluations. Although our theory does not fully cover this class of methods, it is obvious from the order conditions that methods using the $\varphi_{1}$-function only cannot have order larger than two.

\subsection{Exponential multistep methods}

After the discussion of exponential one-step methods, we now turn to exponential multistep methods, which were first introduced by Certaine (1960) and in a more systematic way by Nørsett (1969). The idea of Nørsett was to generalize explicit Adams methods such that they become A-stable and thus suited to stiff problems. Given approximations $u_{j} \approx u\left(t_{j}\right)$, we replace the nonlinearity in (2.1) by its interpolation polynomial at the points

$$
\left(t_{n-k+1}, g\left(t_{n-k+1}, u_{n-k+1}\right)\right), \ldots,\left(t_{n}, g\left(t_{n}, u_{n}\right)\right),
$$

that is,

$$
g\left(t_{n}+\theta h, u\left(t_{n}+\theta h\right)\right) \approx \sum_{j=0}^{k-1}(-1)^{j}\left(\begin{array}{c}
-\theta \\
j
\end{array}\right) \nabla^{j} G_{n}, \quad G_{j}=g\left(t_{j}, u_{j}\right) .
$$

Here, $\nabla^{j} G_{n}$ denotes the $j$ th backward difference defined recursively by

$$
\nabla^{0} G_{n}=G_{n}, \quad \nabla^{j} G_{n}=\nabla^{j-1} G_{n}-\nabla^{j-1} G_{n-1}, \quad j=1,2, \ldots
$$

By inserting this interpolation polynomial into the variation-of-constants formula (2.19), we obtain an explicit exponential Adams method,

$$
u_{n+1}=\mathrm{e}^{-h A} u_{n}+h \sum_{j=0}^{k-1} \gamma_{j}(-h A) \nabla^{j} G_{n},
$$

with weights

$$
\gamma_{j}(z)=(-1)^{j} \int_{0}^{1} \mathrm{e}^{(1-\theta) z}\left(\begin{array}{c}
-\theta \\
j
\end{array}\right) \mathrm{d} \theta .
$$

The weights satisfy the recurrence relation

$$
\begin{aligned}
\gamma_{0}(z) & =\varphi_{1}(z), \\
z \gamma_{k}(z)+1 & =\sum_{j=0}^{k-1} \frac{1}{k-j} \gamma_{j}(z) ;
\end{aligned}
$$

see Nørsett $(1969,(2.9),(2.10))$. For $A=0$, these exponential Adams methods reduce to the well-known explicit Adams methods; see, e.g., Hairer, Nørsett and Wanner (1993, Chapter III).

For an efficient implementation, (2.69) should be reformulated as a corrected exponential Euler step, that is,

$$
u_{n+1}=u_{n}+h \varphi_{1}(-h A)\left(G_{n}-A u_{n}\right)+h \sum_{j=1}^{k-1} \gamma_{j}(-h A) \nabla^{j} G_{n} .
$$


Our error analysis below shows that $\left\|\nabla^{j} G_{n}\right\|=\mathcal{O}\left(h^{j}\right)$ for sufficiently smooth solutions, so it can be expected that Krylov approximations become cheaper with increasing $j$; see also Tokman (2006).

Example 2.26. For $k=1$ we obtain the exponential Euler method (2.29), while for $k=2$ we have

$$
u_{n+1}=u_{n}+h \varphi_{1}(-h A)\left(G_{n}-A u_{n}\right)+h \varphi_{2}(-h A)\left(G_{n}-G_{n-1}\right),
$$

which will be seen to be second-order convergent.

Cox and Matthews (2002) consider the same class of methods, while Calvo and Palencia (2006) constructed and analysed $k$-step methods, where the variation-of-constants formula is taken over an interval of length $k h$ instead of $h$. In contrast to Adams methods, their methods have all parasitic roots on the unit circle. A variety of explicit and implicit schemes is given in Beylkin, Keiser and Vozovoi (1998). Related methods using rational approximation of the arising matrix functions are presented in Lambert and Sigurdsson (1972) and Verwer (1976).

Theorem 2.27. Let the initial value problem (2.1) satisfy Assumptions 2.9 and 2.10, and consider for its numerical solution the $k$-step exponential Adams method (2.69). For $f(t)=g(t, u(t))$ assume that $f \in C^{k}([0, T], X)$. Then, if

$$
\left\|u_{j}-u\left(t_{j}\right)\right\|_{V} \leq c_{0} h^{k}, \quad j=1, \ldots, k-1,
$$

the error bound

$$
\left\|u_{n}-u\left(t_{n}\right)\right\|_{V} \leq C \cdot h^{k} \sup _{0 \leq t \leq t_{n}}\left\|f^{(k)}(t)\right\|
$$

holds uniformly in $0 \leq n h \leq T$. The constant $C$ depends on $T$, but it is independent of $n$ and $h$.

Proof. We will present the proof for the method (2.71), i.e., for $k=2$ only. The proof for $k>2$ is analogous.

Inserting the exact solution into the numerical scheme yields

$$
\begin{aligned}
u\left(t_{n+1}\right)= & \mathrm{e}^{-h A} u\left(t_{n}\right)+h\left(\left(\varphi_{1}(-h A)+\varphi_{2}(-h A)\right) f\left(t_{n}\right)-\varphi_{2}(-h A) f\left(t_{n-1}\right)\right) \\
& +\delta_{n+1}
\end{aligned}
$$

with defect $\delta_{n+1}$. The interpolation error is given by

$$
f\left(t_{n}+\theta h\right)=G_{n}+\theta \nabla G_{n}+\frac{1}{2} h^{2} \theta(\theta+1) f^{\prime \prime}(\zeta(\theta))
$$

for certain intermediate times $\zeta(\theta) \in\left[t_{n-1}, t_{n+1}\right]$. Hence, by the variation- 
of-constants formula (2.8), the defect satisfies

$$
\delta_{n+1}=\frac{1}{2} h^{3} \int_{0}^{1} \mathrm{e}^{-h(1-\theta) A} \theta(\theta+1) f^{\prime \prime}(\zeta(\theta)) \mathrm{d} \theta
$$

and is bounded by

$$
\left\|\delta_{n+1}\right\| \leq C h^{3} M, \quad\left\|\delta_{n+1}\right\|_{V} \leq C h^{2-\alpha} M, \quad M=\sup _{0 \leq t \leq t_{n+1}}\left\|f^{\prime \prime}(t)\right\| .
$$

This yields the error recursion

$$
\begin{aligned}
e_{n+1}=\mathrm{e}^{-h A} e_{n}+h( & \left(\varphi_{1}(-h A)+\varphi_{2}(-h A)\right)\left(G_{n}-f\left(t_{n}\right)\right) \\
& \left.-\varphi_{2}(-h A)\left(G_{n-1}-f\left(t_{n-1}\right)\right)\right)-\delta_{n+1} .
\end{aligned}
$$

From (2.27) we obtain the estimate

$$
\begin{aligned}
\left\|e_{n+1}\right\|_{V} & \leq C\left\|e_{1}\right\|_{V}+C h \sum_{j=0}^{n} \frac{1}{((n+1-j) h)^{\alpha}}\left(\left\|e_{j}\right\|_{V}+h^{2} M\right) \\
& \leq C\left(\left\|e_{1}\right\|_{V}+h^{2} M\right)
\end{aligned}
$$

where the last inequality follows from Gronwall's Lemma 2.15.

\subsection{Linearized exponential multistep methods}

In the same way as for the derivation of exponential Rosenbrock methods in Section 2.4, one can also apply exponential multistep methods (2.69) to the locally linearized equation (2.45). This results in linearized exponential multistep methods.

Example 2.28. A particular two-step method of this type has been proposed in Tokman (2006, (39)):

$$
u_{n+1}=u_{n}+h \varphi_{1}\left(h J_{n}\right) F\left(u_{n}\right)-h \frac{2}{3} \varphi_{2}\left(h J_{n}\right)\left(g_{n}\left(u_{n}\right)-g_{n}\left(u_{n-1}\right)\right) .
$$

It can be interpreted as a perturbed exponential Rosenbrock-Euler step.

Motivated by this example, we consider the variant

$$
u_{n+1}=u_{n}+h \varphi_{1}\left(h J_{n}\right) F\left(u_{n}\right)-2 h \varphi_{3}\left(h J_{n}\right)\left(g_{n}\left(u_{n}\right)-g_{n}\left(u_{n-1}\right)\right) .
$$

For $J_{n}=0$, both methods coincide. The variant (2.72), however, has better convergence properties.

In the subsequent error analysis, we restrict our attention again to autonomous semilinear problems of the form (2.1). This implies that (2.45b) takes the form

$$
J_{n}=-A+\frac{\partial g}{\partial u}\left(u_{n}\right), \quad g_{n}(u(t))=g(u(t))-\frac{\partial g}{\partial u}\left(u_{n}\right) u(t) .
$$


Theorem 2.29. Let the initial value problem (2.1) satisfy Assumptions 2.9, 2.10 , and 2.16 and consider for its numerical solution the linearized exponential two-step method (2.72). For $f(t)=g(u(t))$ assume that $f \in$ $C^{3}([0, T], X)$. Then, if

$$
\left\|u_{1}-u\left(t_{1}\right)\right\|_{V} \leq c_{0} h^{3}
$$

the error bound

$$
\left\|u_{n}-u\left(t_{n}\right)\right\|_{V} \leq C \cdot h^{3} \sup _{0 \leq t \leq t_{n}}\left\|f^{(3)}(t)\right\|
$$

holds uniformly in $0 \leq n h \leq T$. The constant $C$ depends on $T$, but it is independent of $n$ and $h$.

Proof. Let $f_{n}(t)=g_{n}(u(t))$. The exact solution has the expansion

$$
\begin{gathered}
u\left(t_{n+1}\right)=u\left(t_{n}\right)+h \varphi_{1}\left(h J_{n}\right) F\left(u\left(t_{n}\right)\right)+h^{2} \varphi_{2}\left(h J_{n}\right) f_{n}^{\prime}\left(t_{n}\right) \\
+h^{3} \varphi_{3}\left(h J_{n}\right) f_{n}^{\prime \prime}\left(t_{n}\right)+h^{4} \varrho_{3},
\end{gathered}
$$

with a remainder $\varrho_{3}$ as in (2.13) for $p=3$. This remainder satisfies

$$
\left\|\varrho_{3}\right\| \leq C, \quad\left\|\varrho_{3}\right\|_{V} \leq C h^{-\alpha} .
$$

Inserting the exact solution into the numerical scheme yields

$$
\begin{aligned}
u\left(t_{n+1}\right)= & u\left(t_{n}\right)+h \varphi_{1}\left(h J_{n}\right) F\left(u\left(t_{n}\right)\right) \\
& -2 h \varphi_{3}\left(h J_{n}\right)\left(f_{n}\left(t_{n}\right)-f_{n}\left(t_{n-1}\right)\right)+\delta_{n+1},
\end{aligned}
$$

with defect

$$
\delta_{n+1}=h^{2}\left(\varphi_{2}\left(h J_{n}\right)+2 \varphi_{3}\left(h J_{n}\right)\right) f_{n}^{\prime}\left(t_{n}\right)+h^{4} \widetilde{\varrho}_{3}
$$

and a remainder $\widetilde{\varrho}_{3}$ again satisfying (2.74). Due to (2.60), the defects are thus bounded by

$$
\left\|\delta_{n+1}\right\| \leq C\left(h^{2}\left\|e_{n}\right\|_{V}+h^{4}\right), \quad\left\|\delta_{n+1}\right\|_{V} \leq C\left(h^{2-\alpha}\left\|e_{n}\right\|_{V}+h^{4-\alpha}\right),
$$

where $e_{n}=u_{n}-u\left(t_{n}\right)$ denotes the error. This error satisfies the recursion

$$
\begin{gathered}
e_{n+1}=\mathrm{e}^{h J_{n}} e_{n}+h\left(\varphi_{1}\left(h J_{n}\right)-2 \varphi_{3}\left(h J_{n}\right)\right)\left(g_{n}\left(u_{n}\right)-f_{n}\left(t_{n}\right)\right) \\
+2 h \varphi_{3}\left(h J_{n}\right)\left(g_{n}\left(u_{n-1}\right)-f_{n}\left(t_{n-1}\right)\right)-\delta_{n+1},
\end{gathered}
$$

so that, by (2.27) (for $-J_{n}$ instead of $A$ ) and by the stability result of Hochbruck et al. (2009c, Appendix A),

$$
\left\|e_{n}\right\|_{V} \leq C\left\|e_{1}\right\|_{V}+C h \sum_{j=0}^{n-1} \frac{1}{t_{n-j}^{\alpha}}\left(\left\|e_{j}\right\|_{V}+h^{3}\right) .
$$

The stated error bound then follows from a variant of the Discrete Gronwall Lemma (Lemma 2.15). 
Remark 2.30. Higher-order linearized exponential multistep methods can be constructed systematically following this approach. We will discuss details elsewhere.

\subsection{Exponential general linear methods}

In this section we study explicit exponential general linear methods for the autonomous semilinear problem (2.1). For given starting values $u_{0}, u_{1}, \ldots$, $u_{k-1}$, the numerical approximation $u_{n+1}$ at time $t_{n+1}, n \geq k-1$, is given by the recurrence formula

$$
\begin{gathered}
u_{n+1}=\mathrm{e}^{-h A} u_{n}+h \sum_{i=1}^{s} b_{i}(-h A) g\left(t_{n}+c_{i} h, U_{n i}\right) \\
+h \sum_{\ell=1}^{k-1} v_{\ell}(-h A) g\left(t_{n-\ell}, u_{n-\ell}\right) .
\end{gathered}
$$

The internal stages $U_{n i}, 1 \leq i \leq s$, are defined by

$$
\begin{gathered}
U_{n i}=\mathrm{e}^{-c_{i} h A} u_{n}+h \sum_{j=1}^{i-1} a_{i j}(-h A) g\left(t_{n}+c_{j} h, U_{n j}\right) \\
+h \sum_{\ell=1}^{k-1} w_{i \ell}(-h A) g\left(t_{n-\ell}, u_{n-\ell}\right) .
\end{gathered}
$$

The coefficient functions $a_{i j}(-h A), w_{i \ell}(-h A), b_{i}(-h A)$ and $v_{\ell}(-h A)$ are linear combinations of the exponential and related $\varphi$-functions.

The preservation of equilibria of (2.1) is guaranteed under the conditions

$$
\begin{aligned}
\sum_{i=1}^{s} b_{i}(-h A)+\sum_{\ell=1}^{k-1} v_{\ell}(-h A) & =\varphi_{1}(-h A), \\
\sum_{j=1}^{i-1} a_{i j}(-h A)+\sum_{\ell=1}^{k-1} w_{i \ell}(-h A) & =c_{i} \varphi_{1}\left(-c_{i} h A\right), \quad 1 \leq i \leq s .
\end{aligned}
$$

Moreover, these conditions ensure the equivalence of our numerical methods for autonomous and non-autonomous problems. We tacitly assume (2.76) to be satisfied and further suppose $w_{1 \ell}=0$, which implies $c_{1}=0$ and thus $U_{n 1}=u_{n}$.

The explicit exponential Runge-Kutta methods considered in Section 2.3 are contained in the method class (2.75) when setting $k=1$. The exponential Adams methods of Section 2.5 result from (2.75) for the special case of a single stage $s=1$.

For the analysis of exponential general linear methods, one proceeds as usual, by inserting the exact solution into the numerical scheme and 
Table 2.4. The exponential general linear method (2.75) in tableau form.

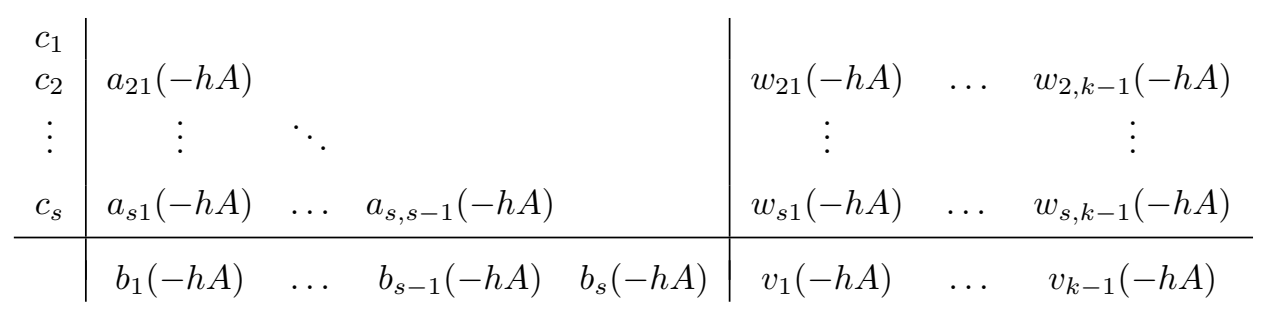

providing bounds for the defects by Taylor expansion within the variationof-constants formula. If we denote the defects for the internal stages $U_{n i}$ by $\Delta_{n i}$ and the defect of the new approximation $u_{n+1}$ by $\delta_{n+1}$, then the numerical scheme (2.75) is said to be of stage order $q$ if $\Delta_{n i}=\mathcal{O}\left(h^{q+1}\right)$ for $1 \leq i \leq s$ and of quadrature order $p$ if $\delta_{n+1}=\mathcal{O}\left(h^{p+1}\right)$. To achieve stage order $q$,

$$
\begin{aligned}
c_{i}^{m} \varphi_{m}\left(c_{i} z\right)= & \sum_{j=1}^{i-1} \frac{c_{j}^{m-1}}{(m-1) !} a_{i j}(z) \\
& +\sum_{\ell=1}^{k-1} \frac{(-\ell)^{m-1}}{(m-1) !} w_{i \ell}(z), \quad 1 \leq i \leq s,
\end{aligned}
$$

has to be satisfied for $1 \leq m \leq q$, and to achieve quadrature order $p$,

$$
\varphi_{m}(z)=\sum_{i=1}^{s} \frac{c_{i}^{m-1}}{(m-1) !} b_{i}(z)+\sum_{\ell=1}^{k-1} \frac{(-\ell)^{m-1}}{(m-1) !} v_{\ell}(z)
$$

must hold for $1 \leq m \leq p$.

The following result from Ostermann, Thalhammer and Wright (2006) shows that for parabolic problems it suffices to satisfy, instead of $(2.77 \mathrm{~b})$ for $m=p$, the weakened quadrature order conditions

$$
\frac{1}{p}=\sum_{i=1}^{s} c_{i}^{p-1} b_{i}(0)+\sum_{\ell=1}^{k-1}(-\ell)^{p-1} v_{\ell}(0),
$$

to obtain the full convergence order $p$, that is, the condition where $m=$ $p$ is fulfilled for $z=0$ only. A similar weakened condition appeared in Theorem 2.17 for exponential Runge-Kutta methods.

Theorem 2.31. Let Assumptions 2.9 and 2.16 be satisfied. Furthermore, suppose that the order conditions (2.77) are valid for $m=1, \ldots, p-1$ and that (2.78) holds. Moreover, let $f$ be $p$-times differentiable with $f^{(p)} \in$ $L^{\infty}(0, T ; X)$ and $A^{\beta} f^{(p-1)}(t) \in X$ for some $0 \leq \beta \leq \alpha$ and for all $0 \leq t \leq T$. 
Then, we have the bound

$$
\begin{aligned}
\left\|u\left(t_{n}\right)-u_{n}\right\|_{V} \leq C & \sum_{m=0}^{k-1}\left\|u\left(t_{m}\right)-u_{m}\right\|_{V}+C h^{p-\alpha+\beta} \sup _{0 \leq t \leq t_{n}}\left\|A^{\beta} f^{(p-1)}(t)\right\| \\
& +C h^{p} \sup _{0 \leq t \leq t_{n}}\left\|f^{(p)}(t)\right\|, \quad t_{k} \leq t_{n} \leq T,
\end{aligned}
$$

uniformly on $0 \leq n h \leq T$, with a constant $C>0$ that is independent of $n$ and $h$.

\section{Lawson and generalized Lawson methods}

A different approach to constructing higher-order exponential methods for semilinear problems (2.1) was proposed by Lawson (1967) and generalized later by Krogstad (2005). Krogstad called the methods (generalized) integrated factor methods. The idea is to use an appropriate transformation of variables, which is motivated by the Adams method (2.69) with a step size $\tau$ :

$$
u\left(t_{n}+\tau\right)=\mathrm{e}^{-\tau A} v(\tau)+\tau \sum_{j=0}^{k-1} \gamma_{j}(-\tau A) \nabla^{j} G_{n}
$$

with weights (2.69b). Let

$$
p(\tau)=\sum_{j=0}^{k-1}(-1)^{j}\left(\begin{array}{c}
-\tau / h \\
j
\end{array}\right) \nabla^{j} G_{n}
$$

denote the interpolation polynomial through $\left(t_{n-k+1}, G_{n-k+1}\right), \ldots,\left(t_{n}, G_{n}\right)$, evaluated at $t_{n}+\tau$. Then, by definition of $\gamma_{j}$, we have

$$
\tau \sum_{j=0}^{k-1} \gamma_{j}(-\tau A) \nabla^{j} G_{n}=\int_{0}^{\tau} \mathrm{e}^{-(\tau-\sigma) A} p(\sigma) \mathrm{d} \sigma .
$$

Differentiating (2.79) with respect to $\tau$ by using (2.81), and inserting the result into (2.1), gives the following differential equation for $v$ :

$$
v^{\prime}(\tau)=\mathrm{e}^{\tau A}\left(g\left(t_{n}+\tau, u\left(t_{n}+\tau\right)\right)-p(\tau)\right),
$$

which is discretized with an explicit Runge-Kutta method or an explicit multistep method, and the result is transformed back to the original variables via (2.79). In the case of Runge-Kutta methods, the resulting methods will be exponential general linear methods. For their analysis see Section 2.7 and Ostermann et al. (2006).

At first glance, this looks strange due to the appearance of $\mathrm{e}^{\tau A}$, which is not defined for semigroups. However, this is only a formal transformation and the factor $\mathrm{e}^{\tau A}$ will eventually disappear. For Runge-Kutta methods, this requires the nodes $c_{i} \in[0,1]$. 
Example 2.32. The method of Lawson (1967) consists of taking $k=0$ (so the sum in (2.79) is empty) and solving (2.82) with Runge's method. This yields the exponential Runge-Kutta method

$$
\begin{array}{c|cc}
0 & & \\
\frac{1}{2} & \frac{1}{2} \mathrm{e}^{-h / 2 A} & \\
\hline & 0 & \mathrm{e}^{-h / 2 A}
\end{array}
$$

which is first-order accurate by Theorem 2.17. Ehle and Lawson (1975) also considered $k=0$, but used higher-order Runge-Kutta methods implemented via rational approximations of the matrix functions.

Example 2.33. Taking $k=1$ and Runge's method gives

\begin{tabular}{c|cc}
0 & & \\
$\frac{1}{2}$ & $\frac{1}{2} \varphi_{1,2}$ & \\
\hline & $\varphi_{1}-\mathrm{e}^{-h / 2 A}$ & $\mathrm{e}^{-h / 2 A}$
\end{tabular},

which is a second-order exponential Runge-Kutta method by Theorem 2.17. For $k=2$ and Runge's method we obtain

$$
\begin{array}{c|cc|c}
0 & & & \\
\frac{1}{2} & \frac{1}{2}\left(\varphi_{1,2}+\varphi_{2,2}\right) & & -\frac{1}{2} \varphi_{2,2} \\
\hline & \varphi_{1}+\varphi_{2}-\frac{3}{2} \mathrm{e}^{-h / 2 A} & \mathrm{e}^{-h / 2 A} & -\varphi_{2}+\frac{1}{2} \mathrm{e}^{-h / 2 A}
\end{array},
$$

which is of second order, by Theorem 2.31 .

Example 2.34. The following scheme, with $k=2$ and the classical fourthorder Runge-Kutta method, was proposed by Krogstad (2005):

$$
\begin{array}{c|cccc|c}
0 & & & & \\
\frac{1}{2} & \frac{1}{2} \varphi_{1,2}+\frac{1}{4} \varphi_{2,2} & & & & -\frac{1}{4} \varphi_{2,2} \\
\frac{1}{2} & \frac{1}{2} \varphi_{1,3}+\frac{1}{4} \varphi_{2,3}-\frac{3}{4} I & \frac{1}{2} I & & & -\frac{1}{4} \varphi_{2,3}+\frac{1}{4} I \\
1 & \varphi_{1,4}+\varphi_{2,4}-\frac{3}{2} \varphi_{0,2} & 0 & \varphi_{0,2} & & -\varphi_{2,4}+\frac{1}{2} \varphi_{0,2} \\
\hline & \varphi_{1}+\varphi_{2}-\varphi_{0,2}-\frac{1}{3} I & \frac{1}{3} \varphi_{0,2} & \frac{1}{3} \varphi_{0,2} & \frac{1}{6} I & -\varphi_{2}+\frac{1}{3} \varphi_{0,2}+\frac{1}{6} I
\end{array} .
$$

This scheme satisfies (2.77) for $m=1,2$ and (2.78) for $p=3$. According to Theorem 2.31, it is of order three.

\subsection{Magnus integrators}

A simple exponential integrator for the non-autonomous linear problem

$$
u^{\prime}(t)=A(t) u(t)+b(t), \quad 0<t \leq T, \quad u(0)=u_{0}
$$

can be obtained by freezing $A$ and $b$ at $t_{n+1 / 2}$. This yields the numerical scheme

$$
u_{n+1}=\mathrm{e}^{h A_{n+1 / 2}} u_{n}+h \varphi_{1}\left(h A_{n+1 / 2}\right) b_{n+1 / 2}, \quad n \geq 0,
$$


with

$$
A_{n+1 / 2}=A\left(t_{n}+\frac{h}{2}\right), \quad b_{n+1 / 2}=b\left(t_{n}+\frac{h}{2}\right) .
$$

The method belongs to the class of Magnus integrators. For $b=0$ it was studied for time-dependent Schrödinger equations in Hochbruck and Lubich (2003); see Section 3.1 below.

For the analysis of scheme (2.84), stability bounds of the form

$$
\left\|\prod_{i=m}^{n} \mathrm{e}^{h A_{i+1 / 2}}\right\|_{X \leftarrow X} \leq C
$$

are essential. We omit the details and refer to González, Ostermann and Thalhammer (2006), where the Magnus integrator (2.84) is shown to be second-order convergent under appropriate regularity assumptions. An extension of this theory to quasi-linear parabolic problems can be found in González and Thalhammer (2007). Higher-order methods for the special case $b=0$ are analysed in Thalhammer (2006).

\section{Highly oscillatory problems}

In this section, we discuss numerical methods for problems having solutions that are highly oscillatory in time. Obviously, the techniques of the previous section, which always used Taylor expansion of the exact solution, are no longer applicable since higher-order time derivatives will now have large norms. In order to obtain error estimates that are useful for practical applications, it is crucial to base the analysis on assumptions related to the particular problem, for instance the assumption that the energy of a physical problem remains bounded.

We will treat first- and second-order differential equations such as Schrödinger equations with time-dependent Hamiltonian, Newtonian equations of motion, and semilinear wave equations. In order to keep this review to a reasonable length, among the many important properties of numerical methods for highly oscillatory problems, we will only address their finitetime error analysis. In particular, we will show that such problems can be solved numerically so that the error of the numerical solution is bounded independently of the highest frequencies arising in the problem. An overview of various principles for the construction of such integrators is given by Cohen, Jahnke, Lorenz and Lubich (2006). We refer to Hairer et al. (2006) and references therein for a detailed study of further properties of the discrete flow, such as long-time behaviour and energy conservation.

The numerical integration of highly oscillatory functions is a strongly related problem. We refer to Iserles and Nørsett (2004) for these quadrature 
methods. Related numerical integrators for highly oscillatory ordinary differential equations are discussed in Iserles (2002a, 2002b); an application to electronic engineering is given in Condon, Deaño and Iserles (2009).

\subsection{Magnus integrators}

This subsection is devoted to the construction of numerical integrators for linear differential equations of the form

$$
\psi^{\prime}(t)=A(t) \psi(t), \quad \psi(0)=\psi_{0},
$$

with a time-dependent, skew-Hermitian matrix $A(t)=-A(t)^{*}$. We follow an approach due to Magnus (1954). In the context of geometric integration, such integrators were studied in Budd and Iserles (1999) and Iserles and Nørsett (1999). An extensive review with many references on Magnus methods is given by Blanes, Casas, Oteo and Ros (2009).

Without loss of generality, we scale the initial state $\psi(0)=\psi_{0}$ such that

$$
\left\|\psi_{0}\right\|=1 .
$$

The assumption that $A$ is skew-Hermitian implies $\|\psi(t)\|=1$ for all $t$. Moreover, we will use the following short notation:

$$
\psi_{n}(\tau)=\psi\left(t_{n}+\tau\right), \quad A_{n}(\tau)=A\left(t_{n}+\tau\right) .
$$

The idea of Magnus consists in writing the solution of (3.1) as

$$
\psi_{n}(\tau)=\exp \left(\Omega_{n}(\tau)\right) \psi_{n}(0), \quad n=0,1, \ldots,
$$

for suitable matrices $\Omega_{n}(\tau)$, which are determined by differentiating (3.2),

$$
\psi_{n}^{\prime}(\tau)=\operatorname{dexp}_{\Omega_{n}(\tau)}\left(\Omega_{n}^{\prime}(\tau)\right) \psi_{n}(0) .
$$

Here, the dexp operator can be expressed by

$$
\operatorname{dexp}_{\Omega}(B)=\varphi_{1}\left(\operatorname{ad}_{\Omega}\right)(B)=\sum_{k \geq 0} \frac{1}{(k+1) !} \operatorname{ad}_{\Omega}^{k}(B),
$$

where $\operatorname{ad}_{\Omega}^{k}$ is defined recursively by

$$
\begin{aligned}
\operatorname{ad}_{\Omega}(B) & =[\Omega, B]=\Omega B-B \Omega, \\
\operatorname{ad}_{\Omega}^{k+1}(B) & =\operatorname{ad}_{\Omega}\left(\operatorname{ad}_{\Omega}^{k}(B)\right), \quad k=1,2, \ldots
\end{aligned}
$$

Hence, $\psi$ defined in (3.2) solves (3.1) if

$$
A_{n}(\tau)=\operatorname{dexp}_{\Omega_{n}(\tau)}\left(\Omega_{n}^{\prime}(\tau)\right), \quad \Omega_{n}(0)=0 .
$$

In order to obtain an explicit differential equation for $\Omega_{n}$, we have to invert the $\operatorname{dexp}_{\Omega_{n}(\tau)}$ operator. Unfortunately, this operator cannot be guaranteed 
to be invertible unless $\left\|\Omega_{n}(\tau)\right\|<\pi$. In this case, with $\beta_{k}$ denoting the $k$ th Bernoulli number, the series

$$
\operatorname{dexp}_{\Omega_{n}(\tau)}^{-1}\left(A_{n}(\tau)\right)=\sum_{k \geq 0} \frac{\beta_{k}}{k !} \operatorname{ad}_{\Omega_{n}(\tau)}^{k}\left(A_{n}(\tau)\right)
$$

converges. Taking the first terms of (3.5) yields

$$
\Omega_{n}^{\prime}(\tau)=A_{n}(\tau)-\frac{1}{2}\left[\Omega_{n}(\tau), A_{n}(\tau)\right]+\frac{1}{12}\left[\Omega_{n}(\tau),\left[\Omega_{n}(\tau), A_{n}(\tau)\right]\right]+\cdots .
$$

Picard iteration yields the Magnus expansion:

$$
\begin{aligned}
\Omega_{n}(h)=\int_{0}^{h} & A_{n}(\tau) \mathrm{d} \tau-\frac{1}{2} \int_{0}^{h}\left[\int_{0}^{\tau} A_{n}(\sigma) \mathrm{d} \sigma, A_{n}(\tau)\right] \mathrm{d} \tau \\
& +\frac{1}{4} \int_{0}^{h}\left[\int_{0}^{\tau}\left[\int_{0}^{\sigma} A_{n}(\mu) \mathrm{d} \mu, A_{n}(\sigma)\right] \mathrm{d} \sigma, A_{n}(\tau)\right] \mathrm{d} \tau \\
& +\frac{1}{12} \int_{0}^{h}\left[\int_{0}^{\tau} A_{n}(\sigma) \mathrm{d} \sigma,\left[\int_{0}^{\tau} A_{n}(\mu) \mathrm{d} \mu, A_{n}(\tau)\right]\right] \mathrm{d} \tau+\cdots .
\end{aligned}
$$

Numerical methods are obtained by truncating this series and approximating the integrals by quadrature rules. If, for instance, $A(t)$ is locally replaced by an interpolation polynomial, then the integrals in the Magnus expansion can be computed analytically. Denoting the result by $\Omega_{n}$, we obtain

$$
\psi_{n+1}=\exp \left(\Omega_{n}\right) \psi_{n}, \quad \Omega_{n} \approx \Omega_{n}(h)
$$

as a numerical approximation to $\psi\left(t_{n+1}\right)$ at $t_{n+1}=t_{n}+h$.

Example 3.1. The simplest method is obtained by truncating the series after the first term and using the midpoint rule for approximating the integral. This yields the exponential midpoint rule

$$
\psi_{n+1}=\mathrm{e}^{h A\left(t_{n}+h / 2\right)} \psi_{n}, \quad n=0,1,2, \ldots,
$$

which corresponds to the choice

$$
\Omega_{n}=h A_{n}(h / 2)=h A\left(t_{n}+h / 2\right)
$$

in (3.7).

Example 3.2. Using two terms of the series and the two-point Gauss quadrature rule with nodes $c_{1,2}=1 / 2 \mp \sqrt{3} / 6$ yields the fourth-order scheme with

$$
\Omega_{n}=\frac{h}{2}\left(A_{n}\left(c_{1} h\right)+A_{n}\left(c_{2} h\right)\right)+\frac{\sqrt{3} h^{2}}{12}\left[A_{n}\left(c_{2} h\right), A_{n}\left(c_{1} h\right)\right] .
$$

This method requires two evaluations of $A$ and one commutator in each time step. 
High-order interpolatory Magnus integrators require the computation of many commutators per step. Blanes, Casas and Ros (2002) constructed (non-interpolatory) Magnus integrators for which the number of commutators could be reduced significantly.

While the Magnus series approach nicely motivates the numerical schemes, it is not at all useful for studying the convergence of Magnus integrators in the case of large $\|h A(t)\|$. The reason is - as mentioned before - that dexp ${\Omega_{n}}_{n}$ need not be invertible and the Magnus expansion need not converge; see Moan and Niesen (2008) for a discussion of the convergence properties of the Magnus expansion. Nevertheless, in important practical applications, Magnus integrators work extremely well even with step sizes for which $\|h A(t)\|$ is large.

For the analysis of the exponential midpoint rule (3.8), we use the following assumption.

Assumption 3.3. We assume that $A(t)$ is skew-Hermitian and that the $m$ th derivative satisfies

$$
\left\|A^{(m)}(t)\right\| \leq M_{m}, \quad m=1,2, \ldots, p
$$

for an appropriate integer $p \geq 1$.

The following theorem was given by Hochbruck and Lubich (1999b).

\section{Theorem 3.4.}

(i) If Assumption 3.3 is satisfied with $p=1$, then the error of the method (3.8) is bounded by

$$
\left\|\psi_{n}-\psi\left(t_{n}\right)\right\| \leq C h
$$

for $0 \leq t_{n} \leq T$. Here, $C$ depends only on $M_{1}$ and $T$.

(ii) Let Assumption 3.3 hold with $p=2$ and let the Hermitian matrix

$$
H(t)=-\mathrm{i} A(t)
$$

be positive definite. Further assume that there exists $0<\alpha \leq 1$ such that

$$
\begin{aligned}
\left\|H(t)^{\alpha} \psi(t)\right\| & \leq C_{\alpha}, \\
\left\|H(t)^{\alpha} A^{\prime}(t) \psi(t)\right\| & \leq C_{\alpha}
\end{aligned}
$$

for $0 \leq t \leq T$. Then the error of the method (3.8) is bounded by

$$
\left\|\psi_{n}-\psi\left(t_{n}\right)\right\| \leq C h^{1+\alpha}
$$

for $0 \leq t_{n} \leq T$. Here, $C$ only depends on $C_{\alpha}, M_{1}, M_{2}$, and $T$.

The assumption of a positive definite $H(t)$ is not essential. If the eigenvalues of $H(t)$ are bounded from below by $-\kappa$, then the result still holds when $H(t)$ is replaced by $H(t)+(\kappa+1) I$ in (3.11) and (3.12). 
Proof of Theorem 3.4. (i) We insert the exact solution into the numerical scheme and obtain

$$
\psi\left(t_{n+1}\right)=\mathrm{e}^{h A_{n}(h / 2)} \psi\left(t_{n}\right)+\delta_{n+1}
$$

with defect $\delta_{n+1}$. By writing (3.1) as

$$
\psi^{\prime}(t)=A(t) \psi(t)=A_{n}(h / 2) \psi(t)+\left(A(t)-A_{n}(h / 2)\right) \psi(t)
$$

and applying the variation-of-constants formula (2.8), we find

$$
\delta_{n+1}=\int_{0}^{h} \mathrm{e}^{(h-\tau) A_{n}(h / 2)}\left(A_{n}(\tau)-A_{n}(h / 2)\right) \psi\left(t_{n}+\tau\right) \mathrm{d} \tau .
$$

By assumption, we have $\left\|\delta_{n+1}\right\| \leq C M_{1} h^{2}$. Denote the error as usual by $e_{n}=\psi_{n}-\psi\left(t_{n}\right)$. Subtracting (3.13) from the numerical scheme (3.8) yields the error recursion

$$
e_{n+1}=\mathrm{e}^{h A_{n}(h / 2)} e_{n}-\delta_{n+1} .
$$

Solving this recursion and using $e_{0}=0$ gives

$$
\left\|e_{n}\right\| \leq \sum_{j=1}^{n}\left\|\delta_{j}\right\| \leq C M_{1} T h .
$$

(ii) Using once more the variation-of-constants formula for $\psi\left(t_{n}+\tau\right)$ in (3.14) yields

$$
\delta_{n+1}=\int_{0}^{h} \mathrm{e}^{(h-\tau) A_{n}(h / 2)}(\tau-h / 2) A_{n}^{\prime}(h / 2) \mathrm{e}^{\tau A_{n}(h / 2)} \psi\left(t_{n}\right) \mathrm{d} \tau+\mathcal{O}\left(h^{3}\right),
$$

where the constant hidden in the $\mathcal{O}\left(h^{3}\right)$ term depends only on $M_{2}$.

In the defect $\delta_{n+1}$, we write $\psi\left(t_{n}\right)=H_{n+1 / 2}^{-\alpha} H_{n+1 / 2}^{\alpha} \psi\left(t_{n}\right)$, where $H_{n+1 / 2}=$ $H\left(t_{n}+h / 2\right)$. From $H(t)=-\mathrm{i} A(t)$ we obtain the bound

$$
\left\|\left(\mathrm{e}^{s A_{n}(h / 2)}-I\right) H_{n}^{-\alpha}\right\| \leq \max _{x>0}\left|\left(\mathrm{e}^{-\mathrm{i} s x}-1\right) / x^{\alpha}\right| \leq C|s|^{\alpha} .
$$

By condition (3.11) we have

$$
\begin{array}{r}
\int_{0}^{h} \mathrm{e}^{(h-\tau) A_{n}(h / 2)}(\tau-h / 2) A_{n}^{\prime}(h / 2)\left(\mathrm{e}^{\tau A_{n}(h / 2)}-I\right) H_{n+1 / 2}^{-\alpha} H_{n+1 / 2}^{\alpha} \psi\left(t_{n}\right) \mathrm{d} \tau \\
=\mathcal{O}\left(h^{2+\alpha}\right),
\end{array}
$$

and therefore the defect can be written as

$$
\delta_{n+1}=\int_{0}^{h} \mathrm{e}^{(h-\tau) A_{n}(h / 2)}(\tau-h / 2) \mathrm{d} \tau A_{n}^{\prime}(h / 2) \psi\left(t_{n}\right)+\mathcal{O}\left(h^{2+\alpha}\right) .
$$

Using condition (3.12) and, once more, the same argument to eliminate the 
factor $\mathrm{e}^{(h-\tau) A_{n}(h / 2)}$, we obtain

$$
\delta_{n+1}=\int_{0}^{h}(\tau-h / 2) \mathrm{d} \tau A_{n}^{\prime}(h / 2) \psi\left(t_{n}\right)+\mathcal{O}\left(h^{2+\alpha}\right)=\mathcal{O}\left(h^{2+\alpha}\right)
$$

because the integral vanishes. This proves the desired estimate.

Remark 3.5. Under certain non-resonance conditions and conditions on the smoothness of the eigen-decompositions of $A(t)$, second-order error bounds can be proved: see Hochbruck and Lubich (1999b, Theorem 2.1) for details.

The convergence behaviour of higher-order Magnus integrators was explained in Hochbruck and Lubich (2003). Our presentation closely follows this paper.

Assumption 3.6. We assume that there exist constants $M_{q}>0$ and a symmetric, positive definite matrix $D$ such that

$$
A(t)=-\mathrm{i}\left(D^{2}+V(t)\right)
$$

with $\left\|V^{(q)}(t)\right\| \leq M_{q}$ for $q=0,1,2, \ldots$ Moreover, we assume that there exists a constant $K>0$ such that, for all vectors $v$ and for all step sizes $h>0$, the commutator bound

$$
\|[A(\tau), A(\sigma)] v\| \leq K h\|D v\| \quad \text { for }|\tau-\sigma| \leq h
$$

holds.

Without loss of generality, we further assume

$$
\|v\| \leq\|D v\| \text { for all } v
$$

This can be achieved by shifting $D$ by the identity matrix.

Example 3.7. We consider the linear Schrödinger equation on a $d$-dimensional torus $\mathcal{T}$ with time-dependent potential,

$$
\mathrm{i} \psi^{\prime}=H(t) \psi=-\Delta \psi+V(t) \psi .
$$

Here $\Delta$ denotes the Laplacian and $V(t)$ is a bounded and smooth multiplication operator. By setting $D^{2}=-\Delta+I$, we obtain

$$
\|D v\|^{2}=\int_{\mathcal{T}}|\nabla v|^{2} \mathrm{~d} x+\int_{\mathcal{T}} v^{2} \mathrm{~d} x
$$

so that $\|D v\|$ is the familiar $H^{1}$ Sobolev norm of $v$. In the spatially discretized case, $\|D v\|$ can be viewed as a discrete Sobolev norm. For a space discretization with minimal grid spacing $\Delta x$, we note that $\left\|D^{2}\right\| \sim \Delta x^{-2}$ and $\|D\| \sim \Delta x^{-1}$. 
In the continuous case, the commutator bound $(3.16)$ is valid since $\left[D^{2}, V\right]$ is a first-order differential operator. For a spectral discretization the commutator bound is proved, uniformly in the discretization parameter, in Jahnke and Lubich (2000, Lemma 3.1).

The idea of Hochbruck and Lubich (2003) was to interpret the numerical approximation as the exact solution of a modified problem. Truncation of the Magnus expansion (3.6) amounts to using a modified $\widetilde{\Omega}_{n}$ instead of $\Omega_{n}$ in $(3.2)$, i.e.,

$$
\widetilde{\psi}_{n}(\tau)=\exp \left(\widetilde{\Omega}_{n}(\tau)\right) \psi\left(t_{n}\right)
$$

For the exponential midpoint rule we truncate the series after the first term and obtain

$$
\widetilde{\Omega}_{n}(\tau)=\int_{0}^{\tau} A_{n}(\sigma) \mathrm{d} \sigma, \quad 0 \leq \tau \leq h .
$$

By differentiating (3.18), we obtain the approximate solution $\widetilde{\psi}_{n}$ as the solution of the modified differential equation

$$
\widetilde{\psi}_{n}^{\prime}(\tau)=\widetilde{A}_{n}(\tau) \widetilde{\psi}_{n}(\tau), \quad \widetilde{\psi}_{n}(0)=\psi_{n}(0)=\psi\left(t_{n}\right),
$$

with

$$
\widetilde{A}_{n}(\tau)=\operatorname{dexp}_{\widetilde{\Omega}_{n}(\tau)}\left(\widetilde{\Omega}_{n}^{\prime}(\tau)\right) .
$$

Note that the truncated Magnus series $\widetilde{\Omega}_{n}(\tau)$ and the modified operator $\widetilde{A}_{n}(\tau)$ are skew-Hermitian if $A(t)$ is skew-Hermitian. As the following lemma shows, a bound on $\widetilde{A}_{n}(\tau)-A_{n}(\tau)$ then immediately leads to a local error bound.

Lemma 3.8. Let $\psi$ be a solution of (3.1) with skew-Hermitian $A(t)$, and $\widetilde{\psi}$ a solution of (3.20). Then their difference is bounded by

$$
\left\|\widetilde{\psi}_{n}(\tau)-\psi_{n}(\tau)\right\| \leq C h^{3} \max _{0 \leq \sigma \leq h}\left\|D \psi_{n}(\sigma)\right\|, \quad 0 \leq \tau \leq h .
$$

Proof. For $E_{n}(\tau)=\widetilde{A}_{n}(\tau)-A_{n}(\tau)$, we write (3.1) as

$$
\psi_{n}^{\prime}(\tau)=A_{n}(\tau) \psi_{n}(\tau)=\widetilde{A}_{n}(\tau) \psi_{n}(\tau)-E_{n}(\tau) \psi_{n}(\tau)
$$

and subtract it from (3.20). This shows that the error $\widetilde{e}_{n}(\tau)=\widetilde{\psi}_{n}(\tau)-\psi_{n}(\tau)$ satisfies

$$
\widetilde{e}_{n}^{\prime}(\tau)=\widetilde{A}_{n}(\tau) \widetilde{e}_{n}(\tau)+E_{n}(\tau) \psi_{n}(\tau), \quad \widetilde{e}_{n}(0)=0 .
$$

Taking the inner product with $\widetilde{e}_{n}$ on both sides of (3.21) and noting that $\widetilde{A}_{n}$ is skew-Hermitian, we have

$$
\operatorname{Re}\left\langle\widetilde{e}_{n}^{\prime}, \widetilde{e}_{n}\right\rangle=\operatorname{Re}\left\langle E_{n} \psi_{n}, \widetilde{e}_{n}\right\rangle \leq\left\|E_{n} \psi_{n}\right\|\left\|\widetilde{e}_{n}\right\|
$$


On the other hand, we have

$$
\operatorname{Re}\left\langle\widetilde{e}_{n}^{\prime}, \widetilde{e}_{n}\right\rangle=\frac{1}{2} \frac{\mathrm{d}}{\mathrm{d} \tau}\left\|\widetilde{e}_{n}\right\|^{2}=\left\|\widetilde{e}_{n}\right\| \frac{\mathrm{d}}{\mathrm{d} \tau}\left\|\widetilde{e}_{n}\right\| \leq\left\|E_{n} \psi_{n}\right\|\left\|\widetilde{e}_{n}\right\| .
$$

Integrating the inequality proves the bound

$$
\left\|\widetilde{\psi}_{n}(\tau)-\psi_{n}(\tau)\right\| \leq \int_{0}^{\tau}\left\|\left(\widetilde{A}_{n}(\sigma)-A_{n}(\sigma)\right) \psi_{n}(\sigma)\right\| \mathrm{d} \sigma .
$$

For $\widetilde{\Omega}_{n}=\widetilde{\Omega}_{n}(h)$, we next use the expansion

$$
\widetilde{A}_{n}=\operatorname{dexp}_{\widetilde{\Omega}_{n}}\left(\widetilde{\Omega}_{n}^{\prime}\right)=\widetilde{\Omega}_{n}^{\prime}+\varphi_{2}\left(\operatorname{ad}_{\widetilde{\Omega}_{n}}\right)\left(\operatorname{ad}_{\widetilde{\Omega}_{n}}\left(\widetilde{\Omega}_{n}^{\prime}\right)\right)
$$

and $A_{n}=\widetilde{\Omega}_{n}^{\prime}$ by (3.19). It was shown in Hochbruck and Lubich (2003, Lemma 5.1) that the remainder can be bounded by

$$
\left\|\varphi_{2}\left(\operatorname{ad}_{\widetilde{\Omega}_{n}}\right)\left(\operatorname{ad}_{\widetilde{\Omega}_{n}}\left(\widetilde{\Omega}_{n}^{\prime}\right)\right) \psi_{n}(\sigma)\right\| \leq C h^{2}\left\|D \psi_{n}(\sigma)\right\|, \quad 0 \leq \tau \leq h .
$$

This finally proves the desired result.

Theorem 3.9. Let Assumption 3.6 hold. If $A(t)$ satisfies the commutator bound (3.16), then the error of the exponential midpoint rule (3.8) is bounded by

$$
\left\|\psi_{n}-\psi\left(t_{n}\right)\right\| \leq C h^{2} t_{n} \max _{0 \leq t \leq t_{n}}\|D \psi(t)\| .
$$

The constant $C$ depends only on $M_{m}$ for $m \leq 2$ and on $K$. In particular, $C$ is independent of $n, h$, and $\|D\|$.

Proof. Inserting the exact solution $\psi$ of (3.1) into the numerical scheme yields

$$
\psi\left(t_{n+1}\right)=\exp \left(\Omega_{n}\right) \psi\left(t_{n}\right)+\delta_{n+1}
$$

with defect

$$
\delta_{n+1}=\psi\left(t_{n+1}\right)-\exp \left(\widetilde{\Omega}_{n}\right) \psi\left(t_{n}\right)+\exp \left(\widetilde{\Omega}_{n}\right) \psi\left(t_{n}\right)-\exp \left(\Omega_{n}\right) \psi\left(t_{n}\right) .
$$

Since the midpoint rule is of order two and due to $\left\|A^{\prime \prime}(t)\right\| \leq M_{2}$, the quadrature error is bounded by

$$
\left\|\widetilde{\Omega}_{n}-\Omega_{n}\right\|=\left\|\int_{0}^{h}\left(A_{n}(\tau)-A_{n}(h / 2)\right) \mathrm{d} \tau\right\| \leq \frac{1}{24} M_{2} h^{3},
$$

and this leads immediately to

$$
\left\|\mathrm{e}^{\widetilde{\Omega}_{n}}-\mathrm{e}^{\Omega_{n}}\right\|=\left\|\int_{0}^{1} \mathrm{e}^{(1-s) \Omega_{n}}\left(\widetilde{\Omega}_{n}-\Omega_{n}\right) \mathrm{e}^{s \widetilde{\Omega}_{n}} \mathrm{~d} s\right\| \leq \frac{1}{24} M_{2} h^{3} .
$$

By Lemma 3.8, estimates (3.22) and (3.24), and assumption (3.17), we obtain

$$
\left\|\delta_{n+1}\right\| \leq C h^{3} \max _{t_{n} \leq t \leq t_{n+1}}\|D \psi(t)\|
$$


Subtracting (3.23) from (3.7) leads to the error recursion

$$
e_{n+1}=\exp \left(\Omega_{n}\right) e_{n}-\delta_{n+1}
$$

for $e_{n}=\psi_{n}-\psi\left(t_{n}\right)$, and thus

$$
\left\|e_{n}\right\| \leq \sum_{j=1}^{n}\left\|\delta_{j}\right\| .
$$

This proves the stated error estimate.

The following error bound of Hochbruck and Lubich (2003, Theorem 3.2) holds for $p$ th-order interpolatory Magnus integrators, i.e., those based on a $p$ th-order truncation of the Magnus series and polynomial interpolation of $A(t)$ at the nodes of a $p$ th-order quadrature formula.

Theorem 3.10. Let Assumption 3.6 hold and assume that for a method of classical order $p$, which contains commutator products of $A\left(t_{n}+c_{j} h\right)$ with $r$ factors, the commutator bounds

$$
\left\|\left[A\left(\tau_{k}\right),\left[\ldots,\left[A\left(\tau_{1}\right), \frac{\mathrm{d}^{m}}{\mathrm{~d} t^{m}} V\left(\tau_{0}\right)\right]\right] \ldots\right] v\right\| \leq K\left\|D^{k} v\right\|
$$

hold for arbitrary times $\tau_{j}$ for $0 \leq m \leq p$ and $k+1 \leq r p$. Then the $p$ th-order interpolatory Magnus integrators satisfy the error bound

$$
\left\|\psi_{n}-\psi\left(t_{n}\right)\right\| \leq C h^{p} t_{n} \max _{0 \leq t \leq t_{n}}\left\|D^{p-1} \psi(t)\right\|,
$$

for time steps $h$ satisfying $h\|D\| \leq c$. The constant $C$ depends only on $M_{m}$ for $m \leq p$, on $K, c$, and on $p$. In particular, $C$ is independent of $n, h$, and $\|D\|$ as long as $h\|D\| \leq c$.

In Example 3.2, we have $r=2$ for order $p=4$. For all Magnus methods proposed in the literature, $r \leq p-1$ holds.

Adiabatic integrators for time-dependent Schrödinger equations

Next we consider the related singularly perturbed problem

$$
\psi^{\prime}(t)=\frac{1}{\varepsilon} A(t) \psi(t), \quad 0<\varepsilon \ll 1 .
$$

We still impose Assumption 3.3. For background information about the adiabatic theory in quantum dynamics we refer to Teufel (2003).

Solving this problem by the exponential midpoint rule would require time steps of size $h=\mathcal{O}(\varepsilon)$ to achieve reasonable accuracy. Moreover, the convergence bounds of Section 3.1 are not practical here, due to the large factor $1 / \varepsilon$ multiplying the derivatives of $A(t)$.

A different approach to the solution of such problems is based on adiabatic transformations: see Jahnke and Lubich (2003), Jahnke (2004), Hairer et al. 
(2006, Chapter XIV) and references therein. The main idea is to represent the solution in a rotating frame of eigenvectors of $A$. Let

$$
-\mathrm{i} A(t)=H(t)=Q(t) \Lambda(t) Q(t)^{*}, \quad \Lambda(t)=\operatorname{diag}\left(\lambda_{k}(t)\right)
$$

be the eigen-decomposition of the real symmetric matrix $H(t)$. Here, $\lambda_{k}(t)$ are the eigenvalues and $Q(t)$ is the orthogonal matrix of eigenvectors of $H(t)$ depending smoothly on $t$ (unless, possibly, crossings of eigenvalues occur). Then the adiabatic transformation is defined by

$$
\eta(t)=\mathrm{e}^{-\frac{\mathrm{i}}{\varepsilon} \Phi(t)} Q(t)^{*} \psi(t)
$$

where

$$
\Phi(t)=\operatorname{diag}\left(\phi_{j}(t)\right)=\int_{0}^{t} \Lambda(s) \mathrm{d} s .
$$

Differentiation with respect to $t$ leads to the differential equation

$$
\eta^{\prime}(t)=\mathrm{e}^{-\frac{\mathrm{i}}{\varepsilon} \Phi(t)} W(t) \mathrm{e}^{\frac{\mathrm{i}}{\varepsilon}(t)} \eta(t), \quad W(t)=Q^{\prime}(t)^{*} Q(t) .
$$

The orthogonality of $Q$ ensures that $W$ is a skew-symmetric matrix for all $t$. Note that the assumption on the smoothness of the eigenvectors guarantees that $\left\|\eta^{\prime}(t)\right\|$ is bounded. The quantum-adiabatic theorem of Born and Fock (1928) yields that the solution of (3.30) satisfies

$$
\eta(t)=\eta(0)+\mathcal{O}(\varepsilon)
$$

uniformly on bounded time intervals if the eigenvalues are separated from each other by a constant $\delta$, which is independent of $\varepsilon$, i.e., if

$$
\left|\lambda_{j}(t)-\lambda_{k}(t)\right| \geq \delta
$$

and if $\left\|\Lambda^{\prime}(t)\right\| \leq C,\left\|Q^{\prime}(t)\right\| \leq C$ for some constant $C$ for $0 \leq t \leq T$. Quantities satisfying (3.31) are called adiabatic invariants.

We consider (3.30) with the bounded matrix

$$
B(t)=\mathrm{e}^{-\frac{\mathrm{i}}{\varepsilon} \Phi(t)} W(t) \mathrm{e}^{\frac{\mathrm{i}}{\varepsilon} \Phi(t)} .
$$

Since $B$ is highly oscillatory, the exponential midpoint rule should not be applied directly. Instead, an averaging process should first be applied. We will explain the basic idea presented by Jahnke and Lubich (2003) and Jahnke (2004) for the construction of such methods. For the simplest scheme - which, however, is not of practical interest - the phase is approximated by

$$
\Phi\left(t_{n}+\tau\right) \approx \Phi\left(t_{n}+h / 2\right)+(h / 2-\tau) \Lambda\left(t_{n}+h / 2\right)=: \widetilde{\Phi}_{n+1 / 2}(\tau),
$$

with an error of size $\mathcal{O}\left(h^{2}\right)$ for $0 \leq \tau \leq h$. This approximation is inserted into $B(t)$, the slow variable $W$ is approximated by its value at the midpoint, and the time average is taken over a time step. This results in the following 
approximation:

$$
B\left(t_{n}+h / 2\right) \approx \widetilde{B}_{n+1 / 2}=\frac{1}{h} \int_{0}^{h} \mathrm{e}^{-\frac{\mathrm{i}}{\varepsilon} \widetilde{\Phi}_{n+1 / 2}(\tau)} W\left(t_{n}+h / 2\right) \mathrm{e}^{\frac{\mathrm{i}}{\varepsilon} \widetilde{\Phi}_{n+1 / 2}(\tau)} \mathrm{d} \tau .
$$

The integration can be done exactly, leading to

$$
\widetilde{B}_{n+1 / 2}=E\left(\Phi\left(t_{n+1 / 2}\right)\right) \bullet \mathcal{I}\left(\Lambda\left(t_{n+1 / 2}\right)\right) \bullet W\left(t_{n+1 / 2}\right),
$$

where $\bullet$ denotes the entrywise product of matrices. The matrices $E$ and $\mathcal{I}$ are defined by

$$
\begin{aligned}
& E(\Phi)_{j k}=\mathrm{e}^{-\frac{\mathrm{i}}{\varepsilon}\left(\phi_{j}-\phi_{k}\right)}, \\
& \mathcal{I}(\Lambda)_{j k}=\int_{-h / 2}^{h / 2} \mathrm{e}^{-\frac{\mathrm{i}}{\varepsilon} \tau\left(\lambda_{j}-\lambda_{k}\right)} \mathrm{d} \tau=\operatorname{sinc}\left(\frac{h}{2 \varepsilon}\left(\lambda_{j}-\lambda_{k}\right)\right) .
\end{aligned}
$$

The averaged adiabatic exponential midpoint rule finally reads

$$
\eta_{n+1}=\mathrm{e}^{h \widetilde{B}_{n+1 / 2}} \eta_{n}, \quad n=0,1,2, \ldots
$$

Unfortunately, the error of this scheme is $\mathcal{O}(\min \{\varepsilon, h\})$ only, which is not satisfactory because $\eta$ is an adiabatic invariant.

Practically relevant schemes use better approximations of the phase and of the factor $W(s)$, and are combined with recursive usage of the variationof-constants formula or higher-order Magnus integrators. Such methods are presented and analysed in Jahnke and Lubich (2003) and Jahnke (2003, 2004).

\subsection{Second-order differential equations}

We now consider the second-order differential equation

$$
q^{\prime \prime}(t)=f(q(t)), \quad q(0)=q_{0}, q^{\prime}(0)=p_{0},
$$

where we assume that the force $f$ can be decomposed into fast and slow forces:

$$
f=f_{\text {fast }}+f_{\text {slow }} \text {. }
$$

In practice, the fast forces are often cheap to evaluate while slow forces are expensive to compute. Therefore, we are interested in constructing methods that use only one evaluation of the slow force per time step.

We start with the semilinear problem where $f_{\text {fast }}$ is a linear force and where, for simplicity, the slow force is denoted by $g$ :

$$
q^{\prime \prime}(t)=-\Omega^{2} q(t)+g(q(t)), \quad q(0)=q_{0}, q^{\prime}(0)=p_{0} .
$$

$\Omega$ is assumed to be a symmetric positive definite matrix. We are interested in the case where high frequencies occur, i.e., $\|\Omega\| \gg 1$. 
The variation-of-constants formula (2.19) applied to the equivalent firstorder system

$$
\left[\begin{array}{l}
q(t) \\
p(t)
\end{array}\right]^{\prime}=\left[\begin{array}{cc}
0 & I \\
-\Omega^{2} & 0
\end{array}\right]\left[\begin{array}{l}
q(t) \\
p(t)
\end{array}\right]+\left[\begin{array}{c}
0 \\
g(q(t))
\end{array}\right]
$$

yields

$$
\left[\begin{array}{l}
q(t) \\
p(t)
\end{array}\right]=R(t \Omega)\left[\begin{array}{l}
q(0) \\
p(0)
\end{array}\right]+\int_{0}^{t}\left[\begin{array}{c}
\Omega^{-1} \sin ((t-s) \Omega) \\
\cos ((t-s) \Omega)
\end{array}\right] g(q(s)) \mathrm{d} s,
$$

with

$$
R(t \Omega)=\exp \left(t\left[\begin{array}{cc}
0 & I \\
-\Omega^{2} & 0
\end{array}\right]\right)=\left[\begin{array}{cc}
\cos (t \Omega) & \Omega^{-1} \sin (t \Omega) \\
-\Omega \sin (t \Omega) & \cos (t \Omega)
\end{array}\right] .
$$

Numerical methods can be constructed by approximating the integral appropriately, either by using a standard quadrature rule or by approximating the function $g$ and integrating exactly. We consider explicit schemes only.

Example 3.11. Gautschi (1961) developed a number of trigonometric multistep methods that use the above variation-of-constants formula (3.38). A symmetric two-step scheme is obtained by replacing $g(q(s))$ by $g\left(q_{n}\right)$ to approximate $q\left(t_{n} \pm h\right)$. Adding and subtracting yield

$$
q_{n+1}-2 \cos (h \Omega) q_{n}+q_{n-1}=h^{2} \operatorname{sinc}^{2}\left(\frac{h}{2} \Omega\right) g\left(q_{n}\right),
$$

where $\operatorname{sinc} \xi=\sin \xi / \xi$. Approximations to the momenta can be obtained via

$$
p_{n+1}-p_{n-1}=2 h \operatorname{sinc}(h \Omega)\left(-\Omega^{2} q_{n}+g\left(q_{n}\right)\right) .
$$

The same approximation of $g$ within the integral (3.38) leads to the one-step scheme

$$
\left[\begin{array}{l}
q_{n+1} \\
p_{n+1}
\end{array}\right]=R(h \Omega)\left[\begin{array}{l}
q_{n} \\
p_{n}
\end{array}\right]+\frac{h}{2}\left[\begin{array}{c}
h \operatorname{sinc}^{2}\left(\frac{h}{2} \Omega\right) \\
2 \operatorname{sinc}(h \Omega)
\end{array}\right] g\left(q_{n}\right) .
$$

However, we would like to stress that the two schemes are not equivalent.

Example 3.12. Deuflhard (1979) refined Gautschi's method by using the trapezoidal rule to approximate the integrals. He suggested the two-step scheme

$$
q_{n+1}-2 \cos (h \Omega) q_{n}+q_{n-1}=h^{2} \operatorname{sinc}(h \Omega) g\left(q_{n}\right)
$$

and its one-step formulation

$$
\left[\begin{array}{l}
q_{n+1} \\
p_{n+1}
\end{array}\right]=R(h \Omega)\left[\begin{array}{l}
q_{n} \\
p_{n}
\end{array}\right]+\frac{h}{2}\left[\begin{array}{c}
h \operatorname{sinc}(h \Omega) g\left(q_{n}\right) \\
\cos (h \Omega) g\left(q_{n}\right)+g\left(q_{n+1}\right)
\end{array}\right] .
$$

In contrast to Gautschi's method, this scheme is symmetric as a one- and a two-step method. 
If $\|\Omega\| \gg 1$, the solution of (3.36) will have highly oscillatory components. The pointwise evaluation of the right-hand side $g$ in combination with large time steps is not advisable. Much better behaviour can be expected if $g$ is evaluated at an averaged value of $q$. Clearly, there is a lot of freedom in choosing such averages. García-Archilla, Sanz-Serna and Skeel (1998) propose solving an auxiliary problem,

$$
y^{\prime \prime}=-\Omega^{2} y, \quad y(0)=q, y^{\prime}(0)=0,
$$

and computing the averaged solutions over a time step of length $h$,

$$
a(q)=\frac{1}{h} \int_{0}^{h} y(\tau) \mathrm{d} \tau=\operatorname{sinc}(h \Omega) q .
$$

The examples above and the averaging approach suggest the following class of two-step methods for the solution of (3.36):

$$
q_{n+1}-2 \cos (h \Omega) q_{n}+q_{n-1}=h^{2} \psi(h \Omega) g\left(\phi(h \Omega) q_{n}\right) .
$$

The schemes are symmetric if the filter functions $\psi$ and $\phi$ are even, which we will assume henceforth.

We also consider the corresponding class of one-step schemes given by

$$
\left[\begin{array}{l}
q_{n+1} \\
p_{n+1}
\end{array}\right]=R(h \Omega)\left[\begin{array}{l}
q_{n} \\
p_{n}
\end{array}\right]+\frac{h}{2}\left[\begin{array}{c}
h \Psi g\left(\Phi q_{n}\right) \\
\Psi_{0} g\left(\Phi q_{n}\right)+\Psi_{1} g\left(\Phi q_{n+1}\right)
\end{array}\right],
$$

where

$$
\Phi=\phi(h \Omega), \quad \Psi=\psi(h \Omega), \quad \Psi_{0}=\psi_{0}(h \Omega), \quad \Psi_{1}=\psi_{1}(h \Omega)
$$

with suitable functions $\phi, \psi, \psi_{0}$ and $\psi_{1}$; see Hairer et al. (2006, Chapter XIII). The one-step method (3.42) is symmetric if and only if

$$
\psi(\xi)=\psi_{1}(\xi) \operatorname{sinc} \xi, \quad \psi_{0}(\xi)=\psi_{1}(\xi) \cos \xi .
$$

A symmetric one-step scheme is equivalent to its corresponding two-step scheme for appropriate initial values.

\section{Finite-time error analysis}

We now study the error of methods of the class (3.42) over a finite time interval $[0, T]$ for problems whose solutions satisfy a finite-energy condition. Our presentation is based on Grimm and Hochbruck (2006).

Assumption 3.13. We assume that there exists a constant $K>0$ such that the solution of (3.37) satisfies the finite-energy condition

$$
\frac{1}{2}\|p(t)\|^{2}+\frac{1}{2}\|\Omega q(t)\|^{2} \leq K^{2}
$$

uniformly for $0 \leq t \leq T$. 
The even analytic functions defining the integrator (3.42) are assumed to be bounded on the non-negative real axis, i.e.,

$$
\max _{\xi \geq 0}|\chi(\xi)| \leq M_{1}, \quad \chi=\phi, \psi, \psi_{0}, \psi_{1},
$$

for some constant $M_{1}$. Moreover, we assume $\phi(0)=1$ and thus the existence of a constant $M_{2}$ such that

$$
\max _{\xi \geq 0}\left|\frac{\phi(\xi)-1}{\xi}\right| \leq M_{2} .
$$

In addition, we assume

$$
\max _{\xi \geq 0}\left|\frac{1}{\sin \frac{\xi}{2}}\left(\operatorname{sinc}^{2} \frac{\xi}{2}-\psi(\xi)\right)\right| \leq M_{3}
$$

and

$$
\max _{\xi \geq 0}\left|\frac{1}{\xi \sin \frac{\xi}{2}}(\operatorname{sinc} \xi-\chi(\xi))\right| \leq M_{4}, \quad \chi=\phi, \psi_{0}, \psi_{1} .
$$

The assumptions made so far are necessary to prove second-order error bounds for the positions $q_{n} \approx q\left(t_{n}\right)$. In order to verify first-order error bounds for the momenta $p$, we assume that

$$
\max _{\xi \geq 0}|\xi \psi(\xi)| \leq M_{5}, \quad \max _{\xi \geq 0}\left|\frac{\xi}{\sin \frac{\xi}{2}}\left(\operatorname{sinc}^{2} \frac{\xi}{2}-\psi(\xi)\right)\right| \leq M_{6} .
$$

Clearly, the constants $M_{1}$ to $M_{6}$ only depend on the choice of the analytic functions. It is easy to find analytic functions for which

$$
M:=\max _{i=1, \ldots, 6} M_{i}
$$

is a small constant.

Example 3.14. The method of Gautschi (1961) described in Example 3.11 uses the filters

$$
\phi(\xi)=1, \quad \psi(\xi)=\operatorname{sinc}^{2}\left(\frac{1}{2} \xi\right), \quad \psi_{0}(\xi)=2 \operatorname{sinc} \xi, \quad \psi_{1}(\xi)=0 .
$$

Hence, condition (3.48) is not satisfied for $\psi_{0}$ and $\psi_{1}$. For the method of Deuflhard (1979), we have

$$
\phi(\xi)=1, \quad \psi(\xi)=\operatorname{sinc} \xi, \quad \psi_{0}(\xi)=\cos \xi, \quad \psi_{1}(\xi)=1 .
$$

These methods do not use an inner filter $\phi$, and thus suffer from resonances.

Filter functions that fulfil (3.45)-(3.49) have been proposed in the literature. García-Archilla et al. (1998) suggested the choice

$$
\phi(\xi)=\operatorname{sinc} \xi, \quad \psi(\xi)=\operatorname{sinc}^{2} \xi,
$$

and Grimm and Hochbruck (2006) suggested

$$
\phi(\xi)=\operatorname{sinc} \xi, \quad \psi(\xi)=\operatorname{sinc}^{3} \xi .
$$


The following theorem of Grimm and Hochbruck (2006) states that suitably chosen filter functions lead to second-order errors in the positions $q$ and first-order error bounds in the momenta $p$.

Theorem 3.15. In (3.36), let $\Omega$ be an arbitrary symmetric positive semidefinite matrix and let the solution satisfy Assumption 3.13. Moreover, suppose that $g$ and the derivatives $g_{q}$ and $g_{q q}$ are bounded in the Euclidean norm or the norms induced by the Euclidean norm, respectively. If the even analytic functions of the scheme (3.42) satisfy (3.45)-(3.48), then

$$
\left\|q\left(t_{n}\right)-q_{n}\right\| \leq h^{2} C, \quad 0 \leq t_{n}=n h \leq T .
$$

The constant $C$ only depends on $T, K, M_{1}, \ldots, M_{4},\|g\|,\left\|g_{q}\right\|$, and $\left\|g_{q q}\right\|$.

If, in addition, (3.49) holds, then

$$
\left\|p\left(t_{n}\right)-p_{n}\right\| \leq h \widetilde{C}, \quad 0 \leq t_{n}=n h \leq T .
$$

The constant $\widetilde{C}$ only depends on $T, K, M,\|g\|,\left\|g_{q}\right\|$, and $\left\|g_{q q}\right\|$.

Proof. Substitution of the exact solution into the integration scheme (3.42) gives

$$
\begin{aligned}
{\left[\begin{array}{l}
q\left(t_{n+1}\right) \\
p\left(t_{n+1}\right)
\end{array}\right]=} & R(h \Omega)\left[\begin{array}{l}
q\left(t_{n}\right) \\
p\left(t_{n}\right)
\end{array}\right] \\
& +\left[\begin{array}{c}
\frac{1}{2} h^{2} \Psi g\left(\Phi q\left(t_{n}\right)\right) \\
\frac{h}{2}\left(\Psi_{0} g\left(\Phi q\left(t_{n}\right)\right)+\Psi_{1} g\left(\Phi q\left(t_{n+1}\right)\right)\right)
\end{array}\right]+\left[\begin{array}{l}
\delta_{n+1} \\
\delta_{n+1}^{\prime}
\end{array}\right],
\end{aligned}
$$

with the defects $\delta_{n+1}$ and $\delta_{n+1}^{\prime}$. Subtraction of equation (3.42) and summation leads to

$$
\begin{gathered}
{\left[\begin{array}{l}
e_{n+1} \\
e_{n+1}^{\prime}
\end{array}\right]=R(h \Omega)^{n+1}\left[\begin{array}{l}
e_{0} \\
e_{0}^{\prime}
\end{array}\right]+\sum_{j=0}^{n} R(h \Omega)^{n-j}\left[\begin{array}{c}
\frac{1}{2} h^{2} \Psi F_{j} e_{j} \\
\frac{1}{2} h \Psi_{0} F_{j} e_{j}+\frac{1}{2} h \Psi_{1} F_{j+1} e_{j+1}
\end{array}\right]} \\
+\left[\begin{array}{c}
\Delta_{n+1} \\
\Delta_{n+1}^{\prime}
\end{array}\right],
\end{gathered}
$$

where $e_{n}:=q\left(t_{n}\right)-q_{n}$ and $e_{n}^{\prime}:=p\left(t_{n}\right)-p_{n}$,

$$
F_{n}:=\int_{0}^{1} g_{q}\left(\Phi\left(q_{n}+\theta e_{n}\right)\right) \mathrm{d} \theta \Phi, \quad\left\|F_{n}\right\| \leq M_{1}\left\|g_{q}\right\|,
$$

and

$$
\left[\begin{array}{c}
\Delta_{n} \\
\Delta_{n}^{\prime}
\end{array}\right]=\sum_{j=1}^{n} R((n-j) h \Omega)\left[\begin{array}{l}
\delta_{j} \\
\delta_{j}^{\prime}
\end{array}\right] .
$$

Unfortunately, the defects $\delta_{j}$ are of size $\mathcal{O}\left(h^{2}\right)$ and the defects $\delta_{j}^{\prime}$ are of size $\mathcal{O}(h)$ only. Bounding the sums in a standard way would yield first-order error bounds for the positions and nothing for the velocities. The key point 
of the proof is now to write

$$
\delta_{n+1}=h^{2} \cdot(\text { highly oscillatory function }) \cdot g\left(\Phi q_{n}\right)+\mathcal{O}\left(h^{3}\right),
$$

and to use a similar but more complicated form for $h \delta_{n+1}^{\prime}$. Such expressions are derived in Grimm and Hochbruck (2006, Lemmas 1 and 2). Using summation by parts, the sums of highly oscillatory functions are bounded independently of $n$ as long as no resonances occur, while the differences of the $g$-function yield an additional factor of $h$ due to the smoothness of $g$. In general, resonant frequencies will deteriorate the convergence and this is exactly the place where the filter functions come into play. They are constructed in such a way that possible resonances disappear. The analysis is detailed in Grimm and Hochbruck (2006, Lemmas 3-6). We then have

$$
\left\|\Delta_{n}\right\| \leq C h^{2} \text { and }\left\|\Delta_{n}^{\prime}\right\| \leq C h,
$$

with a constant $C$ independent of $n$ as long as $0 \leq n h \leq T$. Due to $e_{0}=e_{0}^{\prime}=0$, the recursion (3.51) reads

$$
e_{n}=h \sum_{j=1}^{n-1} L_{j} e_{j}+\Delta_{n}
$$

where

$$
\begin{aligned}
L_{j}:=\frac{1}{2}\left(h \cos ((n-j) h \Omega) \Psi+(n-j) h \operatorname{sinc}((n-j) h \Omega) \Psi_{0}\right. \\
\left.+(n+1-j) h \operatorname{sinc}((n+1-j) h \Omega) \Psi_{1}\right) F_{j} .
\end{aligned}
$$

This yields

$$
\left\|L_{j}\right\| \leq \frac{3}{2} T M_{1}^{2}\left\|g_{q}\right\|
$$

so that $\left\|e_{n}\right\| \leq C h^{2}$ follows from Gronwall's Lemma 2.15. Assumption (3.49) and the recursion for $e_{n}^{\prime}$ finally show $\left\|e_{n}^{\prime}\right\| \leq C h$.

Example 3.16. A similar result for two-step methods (3.41) was proved in Hochbruck and Lubich $(1999 c)$. There, it was shown that the choice

$$
\psi(\xi)=\operatorname{sinc}^{2} \frac{\xi}{2}, \quad \phi(\xi)=\left(1+\frac{1}{3} \operatorname{sinc} 2 \frac{\xi}{2}\right) \operatorname{sinc} \xi
$$

yields a method with a small error constant. The conjecture that the error constant is uniform in the number of time steps and the dimension of the problem was proved elegantly in Grimm (2005a).

In Grimm $(2002,2005 b)$ the error analysis is extended to more general problems, where $\Omega=\Omega(t, q)$ varies smoothly, i.e., the first and the second partial derivatives of $\Omega(t, q)$ are bounded. 
Impulse and mollified impulse method

Writing (3.35) as a first-order differential equation, and using Strang splitting applied to

$$
\left[\begin{array}{c}
q^{\prime} \\
p^{\prime}
\end{array}\right]=\left[\begin{array}{c}
p \\
f_{\text {fast }}(q)
\end{array}\right]+\left[\begin{array}{c}
0 \\
f_{\text {slow }}(q)
\end{array}\right],
$$

yields the impulse method, by Grubmüller, Heller, Windemuth and Schulten (1991) and Tuckerman, Berne and Martyna (1992). Following GarcíaArchilla et al. (1998), we state it in the following form:

$$
\begin{aligned}
\text { kick } & p_{n}^{+}=p_{n}+\frac{h}{2} f_{\text {slow }}\left(q_{n}\right), \\
\text { oscillate } & \text { solve } q^{\prime \prime}=f_{\text {fast }}(q) \text { with initial values }\left(q_{n}, p_{n}^{+}\right) \text {over } \\
& \text { a time step of length } h \text { to obtain }\left(q_{n+1}, p_{n+1}^{-}\right), \\
\text {kick } & p_{n+1}=p_{n+1}^{-}+\frac{h}{2} f_{\text {slow }}\left(q_{n+1}\right) .
\end{aligned}
$$

For linear fast forces (3.36), the 'oscillate' step can be computed exactly, leading to

$$
\left[\begin{array}{c}
q_{n+1} \\
p_{n+1}^{-}
\end{array}\right]=R(h \Omega)\left[\begin{array}{c}
q_{n} \\
p_{n}^{+}
\end{array}\right] .
$$

The method is then precisely the scheme of Deuflhard (1979), described in Example 3.12 above. For more general forces $f$, the 'oscillate' step can be done numerically using smaller time steps. This is affordable since the fast forces are usually cheap to evaluate.

It was observed in Biesiadecki and Skeel (1993) that the impulse method also suffers from resonances, which may occur if eigenvalues of $h \Omega$ are integer multiples of $\pi$. They can be circumvented by suitable averaging. When the slow force has a potential $U, f_{\text {slow }}=-\nabla U$, García-Archilla et al. (1998) suggest replacing $U(q)$ by an averaged potential $\bar{U}(q)=U(a(q))$. This leads to a mollified force:

$$
\bar{f}_{\text {slow }}(q)=a^{\prime}(q)^{T} f_{\text {slow }}(a(q)) .
$$

The same technique can then be used in the general case where $f$ does not have a potential. The mollified impulse method of García-Archilla et al. (1998) consists of using the averaged slow force $\bar{f}_{\text {slow }}$ instead of $f_{\text {slow }}$ in the impulse method.

To compute the mollifier, we solve the auxiliary problem

$$
y^{\prime \prime}=f_{\text {fast }}(y), \quad y(0)=q, y^{\prime}(0)=0,
$$

together with the variational equation

$$
Y^{\prime \prime}=f_{\text {fast }}^{\prime}(y(t)) Y, \quad Y(0)=I, Y^{\prime}(0)=0,
$$


and compute the averaged solutions over a time step of length $h$,

$$
a(q)=\frac{1}{h} \int_{0}^{h} y(\tau) \mathrm{d} \tau, \quad a^{\prime}(q)=\frac{1}{h} \int_{0}^{h} Y(\tau) \mathrm{d} \tau .
$$

Example 3.17. For linear forces as in (3.36), we obtain the averaging function (3.40) and thus $a^{\prime}(q)=\operatorname{sinc}(h \Omega)$. The mollified impulse method then reads:

$$
\begin{aligned}
\text { kick } & p_{n}^{+}=p_{n}+\frac{h}{2} \operatorname{sinc}(h \Omega) g\left(\operatorname{sinc}(h \Omega) q_{n}\right), \\
\text { oscillate } & {\left[\begin{array}{l}
q_{n+1} \\
p_{n+1}^{-}
\end{array}\right]=R(h \Omega)\left[\begin{array}{c}
q_{n} \\
p_{n}^{+}
\end{array}\right], } \\
\text {kick } & p_{n+1}=p_{n+1}^{-}+\frac{h}{2} \operatorname{sinc}(h \Omega) g\left(\operatorname{sinc}(h \Omega) q_{n+1}\right),
\end{aligned}
$$

and this is equivalent to (3.42) with filter (3.50). Thus the convergence is covered by Theorem 3.15. Different proofs have been given by GarcíaArchilla et al. (1998) and by Hochbruck and Lubich (1999c).

\section{Multiple time-stepping}

Yet another way to treat different scales in the general case (3.35) is motivated by the following relation satisfied by the exact solution:

$$
q(t+h)-2 q(t)+q(t-h)=h^{2} \int_{-1}^{1}(1-|\theta|) f(q(t+\theta h)) \mathrm{d} \theta
$$

see Hairer et al. (2006, Chapter VIII.4). The force is approximated by

$$
f\left(q\left(t_{n}+\theta h\right)\right) \approx f_{\text {fast }}(y(\theta h))+f_{\text {slow }}\left(q_{n}\right),
$$

where $y(\tau)$ is a solution of the auxiliary problem

$$
y^{\prime \prime}(\tau)=f_{\text {fast }}(y(\tau))+f_{\text {slow }}\left(q_{n}\right), \quad y(0)=q_{n}, y^{\prime}(0)=p_{n} .
$$

This yields

$$
\int_{-1}^{1}(1-|\theta|) f\left(q_{n}+y(\theta h)\right) \mathrm{d} \theta=\frac{1}{h^{2}}(y(h)-2 y(0)+y(-h)) .
$$

For the velocities we proceed analogously, starting from

$$
y^{\prime}(t+h)-y^{\prime}(t-h)=h \int_{-1}^{1} f(q(t+\theta h)) \mathrm{d} \theta .
$$

The auxiliary problem (3.53) can either be solved exactly or by a standard method such as the Störmer-Verlet scheme with a smaller step size on the time interval $[-h, h]$. Denoting the approximations by $y_{n \pm 1} \approx y( \pm h)$, this 
yields the following symmetric two-step method of Hochbruck and Lubich $(1999 c)$ :

$$
\begin{aligned}
q_{n+1}-2 q_{n}+q_{n-1} & =y_{n+1}-2 y_{n}+y_{n-1}, \\
p_{n+1}-p_{n-1} & =y_{n+1}^{\prime}-y_{n-1}^{\prime} .
\end{aligned}
$$

To obtain a one-step scheme, we solve

$$
y^{\prime \prime}(\tau)=f_{\text {fast }}(y(\tau))+f_{\text {slow }}\left(q_{n}\right), \quad y(0)=q_{n}, y^{\prime}(0)=0 .
$$

Note that, for linear fast forces, the averaged force (3.54) is independent of $y^{\prime}(0)$, as can be seen from (3.38). Moreover, the solution of (3.55) is even, $y(-\tau)=y(\tau)$, which means that the integration has to be done for $\tau \in[0, h]$ only. Hence the averaged force (3.54) can be computed from the solution of (3.55) via

$$
\widetilde{f}_{n}=\frac{2}{h^{2}}\left(y(h)-q_{n}\right) .
$$

This leads to the scheme

$$
\begin{aligned}
p_{n+1 / 2} & =p_{n}+\frac{h}{2} \widetilde{f}_{n}, \\
q_{n+1} & =q_{n}+h p_{n+1 / 2}, \\
p_{n+1} & =p_{n+1 / 2}+\frac{h}{2} \widetilde{f}_{n+1} ;
\end{aligned}
$$

see Hairer et al. (2006, Chapter VIII.4). The variables $p_{n}$ can be interpreted as averaged velocities

$$
p_{n}=\frac{q_{n+1}-q_{n-1}}{2 h} \approx \frac{q\left(t_{n+1}\right)-q\left(t_{n-1}\right)}{2 h}=\frac{1}{2} \int_{-1}^{1} q^{\prime}\left(t_{n}+\theta h\right) \mathrm{d} \theta .
$$

Methods using averaged forces have been used for applications in quantum-classical molecular dynamics. We refer to the review by Cohen et al. (2006) for details and further references.

Remark 3.18. The geometric properties of the methods considered above have recently been intensively studied. It is straightforward to verify that symmetric one-step methods (3.42) are symplectic if and only if

$$
\psi(\xi)=\operatorname{sinc}(\xi) \phi(\xi) .
$$

In Hairer and Lubich (2000), however, long-time near-energy conservation for linear problems has been proved under the condition

$$
\psi(\xi)=\operatorname{sinc}^{2}(\xi) \phi(\xi),
$$

which cannot be satisfied by a symplectic method. For an overview of geometric properties and, in particular, the techniques of modulated Fourier expansions, we refer to Hairer et al. (2006) and Hairer and Lubich (2009). 
Adiabatic integrators

Finally, we consider the singularly perturbed second-order differential equation

$$
q^{\prime \prime}(t)+\frac{1}{\varepsilon^{2}} \Omega(t)^{2} q(t)=\frac{1}{\varepsilon^{2}} f(t),
$$

with real symmetric positive definite matrix $\Omega(t)$. We start with the homogeneous problem and use the notation

$$
\psi(t)=\left[\begin{array}{l}
q(t) \\
p(t)
\end{array}\right]
$$

Rewriting (3.56) as a first-order system reads

$$
\psi^{\prime}(t)=\frac{1}{\varepsilon} A(t) \psi(t)+G(t) \psi(t)
$$

with

$$
A(t)=\left[\begin{array}{cc}
0 & \Omega(t) \\
-\Omega(t) & 0
\end{array}\right], \quad G(t)=\left[\begin{array}{cc}
0 & 0 \\
0 & -\Omega(t)^{-1} \Omega^{\prime}(t)
\end{array}\right] .
$$

Except for a perturbation which is bounded independently of $\varepsilon$, this perturbed first-order problem is of the form (3.27). Next we diagonalize $A$ to obtain (3.28). Note that the eigenvalues of $H$ occur in pairs of $\pm \omega_{j}$, where $\omega_{j}>0$ are the eigenvalues of $\Omega$. Using the adiabatic transformation (3.29) leads to

$$
\eta^{\prime}(t)=\mathrm{e}^{-\frac{\mathrm{i}}{\varepsilon} \Phi(t)} W(t) \mathrm{e}^{\frac{\mathrm{i}}{\varepsilon} \Phi(t)} \eta(t)+\mathrm{e}^{-\frac{\mathrm{i}}{\varepsilon} \Phi(t)} \widetilde{G}(t) \mathrm{e}^{\frac{\mathrm{i}}{\varepsilon} \Phi(t)} \eta(t),
$$

with

$$
W(t)=Q^{\prime}(t)^{*} Q(t) \text { and } \widetilde{G}(t)=Q(t)^{*} G(t) Q(t) .
$$

If, in addition to (3.32), we assume that the frequencies are bounded away from zero,

$$
\left|\lambda_{j}\right| \geq \frac{\delta}{2}
$$

and that $Q$ depends smoothly on $t,(3.57)$ is a linear differential equation with bounded operator. This allows us to apply a Magnus-type integrator, for instance a variant of the exponential midpoint rule (3.34). For details, we refer to Lorenz, Jahnke and Lubich (2005).

The inhomogeneity $f$ in (3.56) can be handled by yet another transformation:

$$
w(t)=q(t)-\Omega(t)^{-2} f(t)+\varepsilon^{2} \Omega(t)^{-2} \frac{\mathrm{d}^{2}}{\mathrm{~d} t^{2}}\left(\Omega(t)^{-2} f(t)\right) .
$$

Differentiating twice shows that $w$ satisfies

$$
w^{\prime \prime}(t)+\frac{1}{\varepsilon^{2}} \Omega(t)^{2} w(t)=\varepsilon^{2} \frac{\mathrm{d}^{2}}{\mathrm{~d} t^{2}}\left(\Omega(t)^{-2} \frac{\mathrm{d}^{2}}{\mathrm{~d} t^{2}}\left(\Omega(t)^{-2} f(t)\right)\right) .
$$


The term on the right-hand side leads to an error of size $\mathcal{O}\left(\varepsilon^{4}\right)$ in the solution, and hence can be omitted if an approximation with error $\mathcal{O}\left(h^{2}\right)$ is required for step sizes $h>\varepsilon$. Then the equation for $w$ is again of the type (3.56).

Adiabatic integrators for mechanical systems with time-dependent frequencies are studied in more detail in Hairer et al. (2006, Chapter XIV.2). In particular, an error analysis for the mollified impulse method for solving singularly perturbed second-order problems is presented there.

\section{Implementation issues}

The implementation of exponential integrators requires the approximation of products of matrix functions with vectors, that is,

$$
\phi(A) v, \quad A \in \mathbb{R}^{d \times d}, \quad v \in \mathbb{R}^{d} .
$$

Clearly, the efficiency of these integrators strongly depends on the numerical linear algebra used to compute these approximations. Standard methods such as diagonalization or Padé approximation will only be useful if the dimension of the matrix is not too large; see Moler and Van Loan (2003) and Higham (2008). We refer to the review by Higham and Al-Mohy (2010) in this volume and concentrate here on large-scale problems.

For simplicity, we always scale $v$ to have unit norm.

\subsection{Chebyshev methods}

If the matrix $A$ is Hermitian or skew-Hermitian for which a priori information about an enclosure of spectrum is available, then a very efficient way of approximating $\phi(A) v$ for some vector $v$ is to use a truncated Chebyshev series. For simplicity, we only consider the exponential function here.

It is well known that a smooth function $\phi:[-1,1] \rightarrow \mathbb{C}$ can be expanded in a Chebyshev series:

$$
\phi(\xi)=c_{0}+2 \sum_{k=1}^{\infty} c_{k} T_{k}(\xi) .
$$

Here, $T_{k}$ denotes the $k$ th Chebyshev polynomial, which is defined by

$$
T_{k}(\xi)=\cos (k \arccos \xi), \quad \xi \in[-1,1]
$$

and

$$
c_{k}=\frac{1}{\pi} \int_{-1}^{1} T_{k}(\xi) \phi(\xi) \frac{1}{\sqrt{1-\xi^{2}}} \mathrm{~d} \xi=\frac{1}{\pi} \int_{0}^{\pi} \cos (k \theta) \phi(\cos \theta) \mathrm{d} \theta .
$$

Chebyshev polynomials satisfy the recursion

$$
T_{k+1}(\xi)=2 \xi T_{k}(\xi)-T_{k-1}(\xi), \quad k=1,2, \ldots
$$

initialized by $T_{0}(\xi)=1$ and $T_{1}(\xi)=\xi$. 
An approximation of $\phi$ can be obtained by truncating the Chebyshev series of $\phi$ after $m$ terms,

$$
S_{m} \phi(\xi)=c_{0}+2 \sum_{k=1}^{m-1} c_{k} T_{k}(\xi)
$$

Note that $S_{m}$ is a polynomial of degree $m-1$.

For real $\omega$ and $\phi(\xi)=\mathrm{e}^{-\omega \xi}$, we have by (4.1)

$$
c_{k}=\mathrm{i}^{k} J_{k}(\mathrm{i} \omega)=I_{k}(-\omega),
$$

where $J_{k}$ denotes the $k$ th Bessel function and $I_{k}$ the $k$ th modified Bessel function, while for $\phi(\xi)=\mathrm{e}^{\mathrm{i} \omega \xi}$ we get

$$
c_{k}=\mathrm{i}^{k} J_{k}(\omega) \text {. }
$$

If $A$ is Hermitian with eigenvalues in $[a, b] \subset \mathbb{R}$, then a linear transformation to the interval $[-1,1]$ yields the approximation

$$
S_{m} \mathrm{e}^{-h A}=\mathrm{e}^{-h(a+b) / 2}\left(c_{0}+2 \sum_{k=1}^{m-1} c_{k} T_{k}\left(\frac{2}{b-a}\left(A-\frac{a+b}{2} I\right)\right)\right),
$$

with

$$
c_{k}=I_{k}\left(-h \frac{b-a}{2}\right)
$$

On the other hand, if $A$ is skew-Hermitian with eigenvalues contained in the interval $\mathrm{i}[a, b]$ on the imaginary axis, then

$$
S_{m} \mathrm{e}^{h A}=\mathrm{e}^{\mathrm{i} h(a+b) / 2}\left(c_{0}+2 \sum_{k=1}^{m-1} c_{k} T_{k}\left(\frac{-2}{b-a}\left(\mathrm{i} A+\frac{a+b}{2} I\right)\right)\right),
$$

with

$$
c_{k}=\mathrm{i}^{k} J_{k}\left(h \frac{b-a}{2}\right) .
$$

The approximation $S_{m} \mathrm{e}^{ \pm h A} v$ can be computed efficiently with the Clenshaw algorithm.

For the approximation error in the case of Hermitian $A$, the following result was given by Stewart and Leyk (1996, Section 4); see also Bergamaschi and Vianello (2000).

Theorem 4.1. Let $A$ be a Hermitian positive semi-definite matrix with eigenvalues in the interval $[0, \rho]$. Then the error of the $m$ th Chebyshev approximation of $\mathrm{e}^{-h A}$, i.e., $\varepsilon_{m}:=\left\|\mathrm{e}^{-h A}-S_{m} \mathrm{e}^{-h A}\right\|$, is bounded by

$$
\varepsilon_{m} \leq 2 \exp \left(-\frac{\beta m^{2}}{h \rho}\right)\left(1+\sqrt{\frac{h \rho \pi}{4 \beta}}\right)+\frac{2 \delta^{h \rho}}{1-\delta}, \quad 0<m \leq h \rho,
$$

where $\beta=2 /(1+\sqrt{5}) \approx 0.618$ and $\delta=\mathrm{e}^{\beta} /(2+\sqrt{5}) \approx 0.438$. 
Remark 4.2. The bounds given in this theorem are not optimal. One can obtain sharper bounds (similar to Theorem 4.4) by combining Bernstein's theorem (see Lubich (2008, Theorem III.2.1)) with the techniques used in the proof of Hochbruck and Lubich (1997, Theorem 2). We do not elaborate on this here.

For skew-Hermitian matrices, the subsequent convergence result is given in Lubich (2008, Theorem III.2.4).

Theorem 4.3. Let $A$ be a skew-Hermitian matrix with eigenvalues in the interval $\mathrm{i}[a, b]$. Then the error of the $m$ th Chebyshev approximation of $\mathrm{e}^{h A}$, i.e., $\varepsilon_{m}:=\left\|\mathrm{e}^{h A}-S_{m} \mathrm{e}^{h A}\right\|$, is bounded by

$$
\varepsilon_{m} \leq 4\left(\exp \left(1-\left(\frac{\omega}{2 m}\right)^{2}\right) \frac{\omega}{2 m}\right)^{m}, \quad \omega=h \frac{b-a}{2} \leq m .
$$

For general matrices, one has to use truncated Faber series instead of Chebyshev series. This technique has been employed by Knizhnerman (1991) and Moret and Novati (2001) for the exponential function.

As mentioned at the start of this subsection, Chebyshev methods require a priori information on the spectrum. In contrast, Krylov subspace methods, which we consider next, work without any a priori information on the field of values of the matrix. Moreover, they exploit clustering of the spectrum and take advantage of particular properties of the vector $b$.

\subsection{Krylov subspace methods}

We assume that the reader is familiar with the basics of solving a linear system with a Krylov subspace method; see, for instance, Saad (2003). The $m$ th Krylov subspace with respect to a vector $b \in \mathbb{C}^{d}$ and a matrix $A \in \mathbb{C}^{d \times d}$ will be denoted by

$$
\mathcal{K}_{m}(A, b)=\operatorname{span}\left\{b, A b, \ldots, A^{m-1} b\right\} .
$$

Without loss of generality we scale $b$ such that $\|b\|=1$.

There are several different ways to derive Krylov approximations to the product of a matrix function with a vector. Here, we present an approach motivated by the Cauchy integral formula:

$$
\phi(A) b=\frac{1}{2 \pi \mathrm{i}} \int_{\Gamma} \phi(\lambda)(\lambda I-A)^{-1} b \mathrm{~d} \lambda=\frac{1}{2 \pi \mathrm{i}} \int_{\Gamma} \phi(\lambda) x(\lambda) \mathrm{d} \lambda .
$$

The curve $\Gamma$ is a suitable contour surrounding the field of values $\mathcal{F}(A)$ of the matrix $A$ and $\phi$ is assumed to be analytic in a neighbourhood of $\mathcal{F}(A)$. The integrand contains, for each $\lambda \in \Gamma$, the solution of a linear system of equations:

$$
(\lambda I-A) x(\lambda)=b .
$$


A Krylov subspace method for approximating the solution of (4.3) first constructs a basis of the Krylov subspace $\mathcal{K}_{m}(\lambda I-A, b)$. Fortunately, since $\mathcal{K}_{m}(A, b)=\mathcal{K}_{m}(\lambda I-A, b)$ for each $\lambda \in \mathbb{C}$, the same Krylov subspace can be used for all $\lambda$.

The Arnoldi method constructs an orthonormal basis $V_{m} \in \mathbb{C}^{d \times m}$ of $\mathcal{K}_{m}(A, b)$ and an unreduced upper Hessenberg matrix $H_{m} \in \mathbb{C}^{m \times m}$ satisfying the standard Krylov recurrence formula

$$
A V_{m}=V_{m} H_{m}+h_{m+1, m} v_{m+1} e_{m}^{T}, \quad V_{m}^{*} V_{m}=I_{m} .
$$

Here $e_{m}$ denotes the $m$ th unit vector in $\mathbb{C}^{d}$. For the shifted systems, we thus have the relation

$$
(\lambda I-A) V_{m}=V_{m}\left(\lambda I-H_{m}\right)-h_{m+1, m} v_{m+1} e_{m}^{T} .
$$

A Galerkin approximation to the solution of (4.3) is defined by requiring that the residual

$$
r_{m}(\lambda)=b-(\lambda I-A) x_{m}(\lambda)
$$

is orthogonal to $\mathcal{K}_{m}(A, b)$. The orthogonality of $V_{m}$ leads to the approximation

$$
x_{m}(\lambda)=V_{m}\left(\lambda I-H_{m}\right)^{-1} e_{1} .
$$

Note that the Galerkin approximation exists for each $m$, since by assumption $\Gamma$ surrounds the field of values of $A$ and thus also $\mathcal{F}\left(H_{m}\right) \subset \mathcal{F}(A)$. An approximation to $\phi(A) b$ is obtained by replacing $x(\lambda) \approx x_{m}(\lambda)$ in (4.2). This yields

$$
\begin{aligned}
\phi(A) b & \approx \frac{1}{2 \pi \mathrm{i}} \int_{\Gamma} \phi(\lambda) x_{m}(\lambda) \mathrm{d} \lambda \\
& =\frac{1}{2 \pi \mathrm{i}} \int_{\Gamma} \phi(\lambda) V_{m}\left(\lambda I-H_{m}\right)^{-1} e_{1} \mathrm{~d} \lambda \\
& =V_{m} \phi\left(H_{m}\right) e_{1},
\end{aligned}
$$

since $V_{m}$ is independent of $\lambda$.

To summarize, the Krylov approximation of $\phi(A) b$ involves two steps. The first is the computation of the basis, for instance using the Arnoldi method, and the second is the computation of $\phi\left(H_{m}\right) e_{1}$, which can be done by standard methods such as diagonalization or Padé approximation (Higham 2008, Higham and Al-Mohy 2010), or methods based on contour integrals (López-Fernández 2009).

The convergence of Krylov subspace approximations has been studied extensively: see, for instance, Druskin and Knizhnerman (1991), Knizhnerman (1991, 1992), Gallopoulos and Saad (1992), Druskin and Knizhnerman (1994, 1995) and Saad (1992). A remarkable property is that the convergence is always superlinear. However, the number of iterations to reach the 
regime of superlinear convergence depends on the geometry of the field of values of the matrix.

The following convergence result for the Hermitian case was given by Hochbruck and Lubich (1997, Theorem 2).

Theorem 4.4. Let $A$ be a Hermitian positive semi-definite matrix with eigenvalues in the interval $[0, \rho]$. Then the error in the Arnoldi approximation of $\mathrm{e}^{-h A} v$, i.e., $\varepsilon_{m}:=\left\|\mathrm{e}^{-h A} v-V_{m} \mathrm{e}^{-h H_{m}} e_{1}\right\|$, is bounded as follows:

$$
\begin{array}{lll}
\varepsilon_{m} & \leq\left(3 \frac{\rho h}{m^{2}}+4 \frac{\sqrt{\rho h}}{m}\right) \mathrm{e}^{-\beta m^{2} /(\rho h)}, & \sqrt{\rho h} \leq m \leq \frac{1}{2} \rho h, \\
\varepsilon_{m} \leq\left(\frac{20}{\rho h}+\frac{6 \sqrt{\pi}}{\sqrt{\rho h}}\right) \mathrm{e}^{(\rho h)^{2} /(16 m)} \mathrm{e}^{-\rho h / 2}\left(\frac{\mathrm{e} \rho h}{4 m}\right)^{m}, & \frac{1}{2} \rho h \leq m,
\end{array}
$$

where $\beta>0.92$.

For the skew-Hermitian case, the regime of superlinear convergence behaviour starts much later, namely with $m \geq\|h A\|$ instead of $m \geq \sqrt{\|h A\|}$ for Hermitian matrices. The result is taken from Hochbruck and Lubich (1997, Theorem 4) in the refined version of Lubich (2008, Theorem III.2.10).

Theorem 4.5. Let $A$ be a skew-Hermitian matrix with eigenvalues contained in an interval $\mathrm{i}[a, b]$ of the imaginary axis. Then, for $\rho=(b-a) / 2$, the error in the Arnoldi approximation of $e^{h A} v$ is bounded by

$$
\varepsilon_{m} \leq 8 \mathrm{e}^{-(\rho h)^{2} /(4 m)}\left(\frac{\mathrm{e} \rho h}{2 m}\right)^{m}, \quad m \geq \rho h .
$$

For $\rho h>1$ we have

$$
\varepsilon_{m} \leq \frac{1}{3}\left(\frac{8}{\rho h}+\frac{11 \sqrt{2}}{\sqrt{\rho h}}\right) \mathrm{e}^{-(\rho h)^{2} /(4 m)}\left(\frac{\mathrm{e} \rho h}{2 m}\right)^{m}, \quad m \geq \rho h .
$$

Additional results for matrices with field of values contained in a disc or a wedge-shaped set can be found in Hochbruck and Lubich (1997). Here we only note that the onset of superlinear convergence behaviour starts with

$$
m \geq(h\|A\|)^{\alpha}, \quad \alpha=\frac{\pi}{2} \frac{1}{\pi-\theta},
$$

where $\theta$ denotes the angle between negative real axis and the boundary of the wedge at the origin. The situation in Theorem 4.4 corresponds to $\theta=0$, while Theorem 4.4 treats the case $\theta=\pi / 2$.

Variants of Krylov subspace methods for the evaluation of matrix functions are an active field of research. Some recent advances such as restarted Krylov subspace methods are given by Eiermann and Ernst (2006) and Niehoff (2007). 


\subsection{Leja interpolation}

Yet a different approach for constructing polynomial approximations is to use interpolation. Since interpolation is ill-conditioned, in general, it is essential to choose the nodes carefully. Caliari, Vianello and Bergamaschi (2004) proposed the use of Leja points for the approximation of the function $\varphi_{1}$ of a matrix whose field of values is contained in an ellipse with foci $(a, 0)$ and $(b, 0)$ on the real axis. In the following, we summarize the basic features of the method. For more details and further references, the reader is referred to Caliari et al. (2004). An extension to $\varphi_{k}$ for $k>1$ is given in Caliari (2007).

A sequence of Leja points $\left\{z_{i}\right\}$ is defined recursively, usually starting from $\left|z_{0}\right|=\max \{|a|,|b|\}$, in such a way that the $(m+1)$ st point $z_{m}$ satisfies

$$
\prod_{i=0}^{m-1}\left|z_{m}-z_{i}\right|=\max _{z \in[a, b]} \prod_{i=0}^{m-1}\left|z-z_{i}\right| .
$$

In practice, Leja points can be extracted from a sufficiently dense set of uniformly distributed points on $[a, b]$. From the definition, it is clear that, in contrast to interpolation in Chebyshev nodes, it is possible to increase the interpolation degree by simply adding new nodes of the same sequence.

Leja points guarantee maximal and superlinear convergence of the interpolant on every ellipse of the confocal family. Therefore, they also ensure superlinear convergence of the corresponding matrix polynomials, provided that the spectrum (or the field of values) of the matrix is contained in one of the above ellipses.

As for Chebyshev approximation, interpolation in Leja points requires a priori information about the field of values of $A$, e.g., by using Gerschgorin's discs. For stability reasons, the method is applied to a scaled and shifted function. Let $c$ and $\gamma$ be defined such that $[a, b]=[c-2 \gamma, c+2 \gamma]$. We interpolate $\varphi_{k}(h(c+\gamma \xi))$ at the Leja points $\left\{\xi_{i}\right\}, \xi_{0}=2$, of the reference interval $[-2,2]$. The latter can be computed once and for all. The matrix Newton polynomial $p_{m}(h A) v$ of degree $m$, which approximates $\varphi_{k}(h A) v$, is then

$$
\begin{aligned}
p_{m}(h A) v & =p_{m-1}(h A) v+d_{m} q_{m}, \\
q_{m} & =\left((A-c I) / \gamma-\xi_{m-1} I\right) q_{m-1},
\end{aligned}
$$

where

$$
p_{0}(h A) v=d_{0} q_{0}, \quad q_{0}=v,
$$

and $\left\{d_{i}\right\}_{i=0}^{m}$ are the divided differences of the function $\varphi_{k}(h(c+\gamma \xi))$ at the points $\left\{\xi_{i}\right\}$. The accurate evaluation of divided differences is considered in Caliari (2007). The method is computationally attractive because of the 
underlying two-term recurrence. When the expected degree $m$ for convergence is too large, an efficient sub-stepping procedure can be used: see Caliari and Ostermann (2009).

\subsection{Contour integrals}

A major disadvantage of polynomial approximations is that the required degree of the polynomials grows with the norm of $A$. If linear systems with coefficient matrix $z I+h A$ can be solved efficiently, rational approximations constitute a good alternative. The most famous example is the approximation of the exponential function on the negative real line, which converges geometrically with rate $1 / 9.28903 \ldots$; see Schmelzer and Trefethen (2007) for a near-best approximation based on the Carathéodory-Fejér procedure, and references therein.

Among the many different approaches, we mention rational Krylov methods. Some recent references are Moret and Novati (2004), Hochbruck and van den Eshof (2006), Frommer and Simoncini (2008) and Grimm and Hochbruck (2008). Extended Krylov subspace methods are investigated in Knizhnerman and Simoncini (2009). It is beyond the scope of this review paper to give a complete list.

Other rational approaches are based on representing the matrix function as an appropriate contour integral: see, e.g., Schmelzer and Trefethen (2007), Trefethen, Weideman and Schmelzer (2006), Schädle, López-Fernández and Lubich (2006), to mention just a few recent articles. Here we present the approach of López-Fernández, Palencia and Schädle (2006), which is based on the inversion of the Laplace transform. Let

$$
\Sigma_{\delta}=\{z \in \mathbb{C}|| \arg (-z) \mid \leq \delta\} \cup\{0\}, \quad 0<\delta<\pi / 2,
$$

be a sector in the left complex half-plane, and let

$$
\Phi: \mathbb{C} \backslash \Sigma_{\delta} \rightarrow X
$$

be a holomorphic function satisfying

$$
\|\Phi(z)\| \leq \frac{M}{|z|}
$$

for some constant $M>0$. It is well known that, in this case, $\Phi$ is the Laplace transform of

$$
\varphi(t)=\frac{1}{2 \pi \mathrm{i}} \int_{\Gamma} \mathrm{e}^{t z} \Phi(z) \mathrm{d} z, \quad|\arg t| \leq \pi / 2-\delta .
$$

Our aim is to reconstruct $\varphi$ from a few evaluations of $\Phi(z)$ on a certain contour $\Gamma$.

For a given $\delta$, we select parameters $d>0, \alpha>0$ satisfying

$$
0<\alpha-d<\alpha+d<\pi / 2-\delta \text {. }
$$


To invert the Laplace transform (4.8), we use the contour formed by the left branch of a hyperbola,

$$
\Gamma=\{\lambda T(x)+\gamma \mid x \in \mathbb{R}\},
$$

where $\lambda>0$ and

$$
T(x)=1-\sin (\alpha+\mathrm{i} x) .
$$

Inserting this contour into (4.8), we obtain

$$
\varphi(t)=\int_{-\infty}^{\infty} G(t, x) \mathrm{d} x
$$

where the function

$$
G(t, w)=-\frac{\lambda T^{\prime}(w)}{2 \pi \mathrm{i}} \mathrm{e}^{t(\lambda T(w)+\gamma)} \Phi(\lambda T(w)+\gamma)
$$

is holomorphic on the horizontal strip $\{w \in \mathbb{C}|| \operatorname{Im} w \mid<d\}$.

For the numerical approximation $\varphi(n, t)$ of $(4.10)$, we consider the quadrature rule

$$
\varphi(n, t)=h \sum_{\ell=-n}^{n} G\left(t, x_{\ell}\right),
$$

with nodes $x_{\ell}=\ell h$. This approximation can be rewritten in terms of $\Phi$ as

$$
\varphi(n, t)=h \sum_{\ell=-n}^{n} w_{\ell} \mathrm{e}^{t z_{\ell}} \Phi\left(z_{\ell}\right),
$$

with weights

$$
w_{\ell}=\frac{h \lambda}{2 \pi \mathrm{i}} T^{\prime}\left(x_{\ell}\right)
$$

and nodes $z_{\ell}=\lambda T\left(x_{\ell}\right)$.

For this approximation, López-Fernández et al. (2006) proved the following error bound.

Theorem 4.6. Let $\Phi$ satisfy (4.7) and let $\alpha, d$ be chosen according to (4.9). Then, for $t_{0}>0, \Lambda \geq 1,0<\theta<1, n \geq 1$, and

$$
a(\theta)=\operatorname{arccosh}\left(\frac{\Lambda}{(1-\theta) \sin \alpha}\right)
$$

the choice

$$
h=\frac{a(\theta)}{n}, \quad \lambda=\frac{2 \pi d n(1-\theta)}{t_{0} \Lambda a(\theta)}
$$

yields the uniform estimate

$$
\|\varphi(t)-\varphi(n, t)\| \leq \frac{4 M}{\pi} \psi\left(\alpha, d, \lambda, t_{0}\right) \frac{\varepsilon_{n}(\theta)^{\theta}}{1-\varepsilon_{n}(\theta)},
$$


for $t_{0} \leq t \leq \Lambda t_{0}$, where

$$
\psi\left(\alpha, d, \lambda, t_{0}\right)=\sqrt{\frac{1+\sin (\alpha+d)}{1-\sin (\alpha+d)}}\left(1+\left|\log \left(1-\mathrm{e}^{-\lambda t_{0} \sin (\alpha-d)}\right)\right|\right)
$$

and

$$
\varepsilon_{n}(\theta)=\exp \left(-\frac{2 \pi d n}{a(\theta)}\right)
$$

López-Fernández (2009) suggests applying these techniques for evaluating the $\varphi$-functions of generators of analytic semigroups. For this purpose, we write

$$
\varphi_{k}(-h A)=\int_{0}^{1} \mathrm{e}^{-h(1-\theta) A} \frac{\theta^{k-1}}{(k-1) !} \mathrm{d} \theta=\mathcal{L}^{-1}\left(\mathcal{L}(\chi(\cdot, h A)) \mathcal{L}\left(\varrho_{k}\right)\right)(1),
$$

where $\mathcal{L}$ denotes the Laplace transform and

$$
\chi(\theta, h A)=\mathrm{e}^{-h \theta A}, \quad \rho_{k}(\theta)=\frac{\theta^{k-1}}{(k-1) !} .
$$

This shows at once that

$$
\Phi_{k}(z,-h A)=\frac{1}{z^{k}}(z I+h A)^{-1} .
$$

If $A$ is a matrix and $v$ is a vector of appropriate dimension, the evaluation of $\Phi_{k}\left(z_{\ell},-h A\right) v$ requires the solution of a linear system of equations.

\subsection{Hints on mathematical software}

Most of the mathematical software for exponential integrators is still in its infancy. Although there exist many experimental codes, only a few of these programs are sufficiently developed and documented for general use. The following packages, however, are well established and much used.

The exponential integrator exp $4,{ }^{1}$ by Hochbruck et al. (1998), is a variable step size implementation of an exponential Rosenbrock-type method, described in Example 2.25. Implementations in both MATLAB and $\mathrm{C}$ are available. The code has an option for dense matrices, where the required matrix functions are computed exactly. For large sparse matrices, however, Krylov subspace methods are employed. The software is well tested and has been successfully used in various applications.

In contrast to the integrator exp4, the MATLAB package EXPINT, ${ }^{2}$ by Berland, Skaflestad and Wright $(2007 b)$, is more of a developing and testing tool for exponential integrators. It is a constant step size implementation of various integrators from the literature, and it is supplemented

${ }^{1}$ http://www.am.uni-duesseldorf.de/en/Research/04_Software.php

${ }^{2}$ http://www.math.ntnu.no/num/expint/matlab.php 
by some numerical examples for the purpose of testing and comparison. The $\varphi$-functions are evaluated by an extension of the well-known scaling and squaring method for the matrix exponential function (Moler and Van Loan 2003, Higham 2008).

The next two packages can be used to compute the product of the matrix exponential and the matrix $\varphi_{1}$-function with a vector, respectively. In this capacity, they are useful building blocks for exponential integrators. Moreover, they can be used for solving first-order linear systems

$$
u^{\prime}(t)+A u(t)=b .
$$

One of these packages is Expokit, ${ }^{3}$ by Sidje (1998). It is available in MATLAB and FORTRAN. The computation of the above-mentioned matrix functions is based on Krylov subspace methods. The other package is SPARSKIT, ${ }^{4}$ by Saad (1994). Designed as a basic toolkit for sparse matrix computations, its routines EXPPRO and PHIPRO provide the product of the matrix exponential and the matrix $\varphi_{1}$-function with a vector, respectively. The computations are again based on Krylov subspace methods.

Finally, we mention the recent MATLAB function phipm, ${ }^{5}$ by Niesen and Wright (2009). It provides the action of matrix $\varphi$-functions, computed by Krylov subspace methods.

\section{Applications}

In recent years, exponential integrators have been employed in various largescale computations. Here we will discuss some typical applications that illustrate the computational benefits of exponential integrators. The given list is by no means exhaustive, and is steadily growing.

Nonlinear partial differential equations with constant coefficients on rectangular domains can be spatially discretized by spectral methods. Integrating the resulting semidiscrete ordinary differential equations with constant step sizes allows us to compute the arising matrix functions once and for all at the beginning of the time integration. Such an approach has been advocated by Kassam and Trefethen (2005), and was successfully used in Klein (2008).

\subsection{Reaction-advection-diffusion equations}

Time-dependent partial differential equations that couple reaction terms with advection and diffusion terms form an important class of (semi)linear parabolic equations. For a recent review on numerical methods for such equations, we refer to the textbook by Hundsdorfer and Verwer (2007).

\footnotetext{
${ }^{3}$ http://www.maths.uq.edu.au/expokit/

${ }^{4}$ http://www-users.cs.umn.edu/ saad/software/SPARSKIT/sparskit.html

${ }^{5}$ http://www.amsta.leeds.ac.uk/ jitse/software.html
} 
Exponential integrators for reaction-diffusion systems are used in Friesner et al. (1989). For linear advection-diffusion problems, Bergamaschi, Caliari and Vianello (2004) and Caliari et al. (2004) consider a finite element discretization with mass lumping. The arising linear differential equation is integrated exactly with an exponential integrator. The required $\varphi_{1}$-function of the non-symmetric stiffness matrix is approximated by interpolation at Leja points. A comparison with the Crank-Nicolson method is given for problems in two and three space dimensions. Bergamaschi, Caliari, Martínez and Vianello (2005) and Martínez, Bergamaschi, Caliari and Vianello (2009) describe parallel implementations of the same approach. Numerical comparisons for a reaction-advection-diffusion equation in two dimensions are given in Caliari and Ostermann (2009). Krylov and Leja approximations for large-scale matrix exponentials are compared in Bergamaschi, Caliari, Martínez and Vianello (2006).

\subsection{Mathematical finance}

Option pricing in mathematical finance is yet another source of parabolic (integro-)differential equations. Tangman, Gopaul and Bhuruth (2008) consider European, barrier and butterfly spread options for the Black-Scholes model and Merton's jump diffusion model. In that paper, the performance of exponential integrators is compared with the traditional Crank-Nicolson method. American options are considered by Rambeerich, Tangman, Gopaul and Bhuruth (2009), and Gondal (2010).

Lee and Sheen (2009) consider a contour integral approach for the solution of the Black-Scholes equations. A hyperbolic contour is used for the numerical inversion of the Laplace transform. In't Hout and Weideman (2009) extend this approach to the Heston model in two dimensions. They consider a parabolic contour and compare the performance of the resulting method with conventional ADI splitting methods.

\subsection{Classical and quantum-classical molecular dynamics}

Molecular dynamics is concerned with the simulation of long-range interaction of molecules. The different time scales involved, together with high oscillations, make these simulations very time-consuming, even on supercomputers. The challenges arising in computational molecular biophysics are well documented in the survey paper by Schlick, Skeel, Brunger, Kalé, Board, Hermans and Schulten (1999).

The mollified impulse method (see Section 3.2) was an important step towards more efficient integrators that allow longer time steps. More details and some applications are given in Izaguirre, Reich and Skeel (1999), Ma, Izaguirre and Skeel (2003) and Ma and Izaguirre (2003a, 2003b).

Exponential integrators have also been proposed for the time integration of mixed quantum-classical models: see Nettesheim, Bornemann, Schmidt 
and Schütte (1996), Schütte and Bornemann (1999), Nettesheim and Schütte (1999), and the collection by Deuflhard, Hermans, Leimkuhler, Mark, Reich and Skeel (1999). These models describe a small but important part of the system by quantum mechanics while the majority of atoms is described by classical mechanics. The quantum-classical molecular dynamics (QCMD) model is a famous example, where a singularly perturbed Schrödinger equation is coupled nonlinearly to classical Newtonian equations of motion. Due to the different time scales in the classical and the quantum evolution, the solutions are typically highly oscillatory. Hochbruck and Lubich (1999b) propose and analyse a variety of methods in which the action of the exponential of the Hamiltonian to a wave function is approximated by the Lanczos algorithm. Methods for more general problems, including the Car-Parinello equations of ab initio molecular dynamics, are presented in Hochbruck and Lubich (1999a). The methods allow the use of step sizes that are much larger than the inverse of the largest frequency in the system.

Numerical integrators for quantum dynamics close to the adiabatic limit are studied in Jahnke and Lubich (2003) and Jahnke (2004). The main idea here is to apply a clever transformation of variables (cf. (3.29)). For the reformulated problem, time-reversible numerical integrators have been proposed, which can use much larger step sizes than standard schemes. A generalization of these techniques to QCMD models can be found in Jahnke (2003) and to linear second-order differential equations with time-varying eigen-decompositions in Lorenz et al. (2005).

\subsection{Schrödinger equations}

Krylov subspace methods and Chebyshev approximations of the matrix exponential operator have a long tradition in computational chemistry and physics: see, e.g., Nauts and Wyatt (1983), Tal-Ezer and Kosloff (1984), Park and Light (1986), Tal-Ezer, Kosloff and Cerjan (1992), Kosloff (1994) and Peskin, Kosloff and Moiseyev (1994).

Berland, Owren and Skaflestad (2006) compare the performance of Lawson methods and exponential Runge-Kutta methods for nonlinear Schrödinger equations, and Berland, Islas and Schober (2007a) use exponential integrators for the cubic nonlinear Schrödinger equation with periodic boundary conditions. Celledoni, Cohen and Owren (2008) derive and study symmetric exponential integrators and present some results on the cubic nonlinear Schrödinger equation.

\subsection{Maxwell equations}

Botchev, Harutyunyan and van der Vegt (2006) suggest using a Gautschitype method (see Section 3.2) combined with Krylov approximations for the solution of a finite element discretization of linear three-dimensional 
Maxwell equations. The paper also includes an analysis of the Krylov approximation error and of the dispersion of the numerical solution. Moreover, comparisons with a standard leap-frog method are reported. Later, Botchev, Faragó and Horváth (2009) presented further comparisons of this method with a variety of splitting methods, and in Verwer and Botchev (2009), the exponential trapezoidal rule described in Example 2.6 is considered.

Tokman and Bellan (2002) present a three-dimensional magnetohydrodynamic (MHD) model for the description of the evolution of coronal magnetic arcades in response to photospheric flows, which is able to reproduce a number of features characteristic of coronal mass ejection observations. For the time integration, the scheme exp4 by Hochbruck et al. (1998), which is a particular variant of the exponential Rosenbrock-type method discussed in Example 2.25, is used.

Karle, Schweitzer, Hochbruck, Laedke and Spatschek (2006) and Karle, Schweitzer, Hochbruck and Spatschek (2008) suggest using Gautschi-type integrators for the simulation of nonlinear wave motion in dispersive media. The model derived in these papers applies to laser propagation in a relativistic plasma. For the one-dimensional problem, the matrix functions are evaluated by fast Fourier transformations, while for a two-dimensional problem this is no longer efficient if the laser pulse propagates within the plasma. The authors propose the use of two-dimensional fast Fourier transformations only in vacuum, where the integrator can take very large time steps. Within the plasma, where smaller time steps are required, a physically motivated spatial splitting is used for the implementation. Comparisons with a standard leap-frog integrator are reported and an implementation on parallel computers is discussed.

Another application is the simulation of electromagnetic wave propagation in optical and photonic systems. Niegemann, Tkeshelashvili and Busch (2007) suggest using an exponential quadrature rule (see Section 2.2) for the solution of linear Maxwell's equations that uses only matrix exponentials instead of linear combinations of $\varphi$-functions as in (2.9). In contrast to the standard finite difference time-domain (FDTD) method, their exponential quadrature rule allows the handling of lossy and anisotropic materials, as well as advanced boundary conditions, such as perfectly matched layers or auxiliary differential equations. For the implementation, Krylov subspace methods are used.

Since many complex optical and photonic systems lead to nonlinear problems, Pototschnig, Niegemann, Tkeshelashvili and Busch (2009) generalize this approach to Maxwell's equations with general nonlinear polarizations. As concrete examples, they investigate instantaneous Kerr nonlinearities, and give an overview of the treatment of coupled systems dynamics. They discuss Lawson methods (see Section 2.7) and a particular case of exponential Rosenbrock methods from Hochbruck et al. (1998) (see Section 2.4). 


\subsection{Regularization of ill-posed problems}

Asymptotic regularization is a well-established tool for treating nonlinear ill-posed problems. Tautenhahn (1994) analysed the convergence of this approach for linear and nonlinear problems.

For its numerical realization, an appropriate numerical method for solving the Showalter differential equation is required. Applications of standard integration schemes yield well-known regularization methods. For example, the explicit Euler method and the linearly implicit Euler method are equivalent to the Landweber method and the Levenberg-Marquardt regularization, respectively. Hochbruck, Hönig and Ostermann (2009a, 2009b) present a variable step size analysis for the exponential Euler method. Optimal convergence rates are achieved under suitable assumptions on the initial error.

\section{Historical remarks}

We conclude this review with some comments on how the concept of exponential integrators has developed historically. However, it is not our intention to provide a full account of the history of exponential integrators. Relevant comments with appropriate references have already been given at various locations in the main text. Additional historical details can be found in Minchev and Wright (2005).

To the best of our knowledge, exponential integrators were first considered by Hersch (1958). The starting point of his investigation was the observation that standard difference schemes for the numerical solution of differential equations do not give the exact solution, in general, even if the differential equation is simple and can be solved by elementary methods. He then proposed new schemes that are exact for linear problems with constant coefficients. His main interest in this work was the study of improved schemes for boundary value and eigenvalue problems with variable coefficients in one or several spatial variables.

Certaine (1960) used the variation-of-constants formula to derive exponential multistep methods for the numerical solution of semilinear initial value problems (2.1). He suggested using the exponential Euler scheme (2.29) as a predictor for implicit exponential one- and two-step methods. Pope (1963) considered general nonlinear problems (2.44) and suggested linearizing them in each time step. The simplest scheme making use of this linearization is the exponential Rosenbrock-Euler method (2.53) for non-autonomous problems.

Gautschi (1961) proposed the use of trigonometric polynomials for the construction of time integration schemes for second-order problems. Probably the most famous example is the two-step method of Example 3.11. Related methods were constructed by Deuflhard (1979), using a theoretical 
approach to extrapolation methods. Example 3.12 contains his two-step method for semilinear second-order differential equations.

Nørsett (1969) used an idea similar to that of Certaine (1960) to construct explicit exponential Adams methods for semilinear problems (2.1). These methods are discussed in Section 2.5. A different idea due to Lawson (1967) consists in using an appropriate transformation of variables. Here, the simplest scheme is obtained by using the exponential function as a transformation: see Example 2.32. The hope is that the transformed equation will be non-stiff and can be discretized efficiently with an explicit Runge-Kutta or multistep method. The resulting approximations are then transformed back to the original variables. The idea was further developed in Ehle and Lawson (1975).

The first exponential Runge-Kutta methods (2.20) were constructed by Lawson (1967) and Ehle and Lawson (1975), with coefficients being exponential functions. Higher-order methods, however, require more $\varphi$-functions. Such methods were first proposed by Friedli (1978).

The term 'exponential integrators' was coined in the seminal paper by Hochbruck, Lubich and Selhofer (1998), which proposed an implementation of the code exp4 (which belongs to the class of Rosenbrock-type methods; see Section 2.4), including adaptive time-stepping combined with Krylov subspace approximations of the $\varphi_{1}$-functions. The class of methods had already been introduced in Hochbruck and Lubich (1997), but it turned out that a naive implementation of exponential integrators would not be efficient in general. The integrator exp4 used a clever construction which minimizes the numerical linear algebra costs. In this way, the first exponential integrator was obtained that was competitive for certain time-dependent partial differential equations.

The paper by Hochbruck et al. (1998) led to a revival of exponential integrators and initiated various activities in different directions on the construction, analysis, implementation, and application of such methods. These activities can be divided into four different groups: integrators for problems with temporally smooth solutions, integrators for problems with highly oscillatory solutions, improvements of the numerical linear algebra, and integrators designed for a specific application. In fact, these four topics correspond to the four main chapters of this review paper.

\section{Acknowledgements}

We thank the members of our groups for their careful reading of this paper and helpful comments. Moreover, we are grateful to Christian Lubich, Valeria Simoncini, and Nick Higham for their comments on an earlier version of this paper.

This work was supported by the Deutsche Forschungsgemeinschaft via SFB TR 18. 


\section{REFERENCES}

L. Bergamaschi and M. Vianello (2000), 'Efficient computation of the exponential operator for large, sparse, symmetric matrices', Numer. Linear Algebra Appl. 7, 27-45.

L. Bergamaschi, M. Caliari and M. Vianello (2004), The ReLPM exponential integrator for FE discretizations of advection-diffusion equations. In Computational Science: ICCS 2004, Vol. 3039 of Lecture Notes in Computer Science, Springer, pp. 434-442.

L. Bergamaschi, M. Caliari, A. Martínez and M. Vianello (2005), A parallel exponential integrator for large-scale discretizations of advection-diffusion models. In Recent Advances in Parallel Virtual Machine and Message Passing Interface, Vol. 3666 of Lecture Notes in Computer Science, Springer, pp. 483-492.

L. Bergamaschi, M. Caliari, A. Martínez and M. Vianello (2006), Comparing Leja and Krylov approximations of large scale matrix exponentials. In Computational Science: ICCS 2006, Vol. 3994 of Lecture Notes in Computer Science, Springer, pp. 685-692.

H. Berland, A. L. Islas and C. M. Schober (2007a), 'Conservation of phase space properties using exponential integrators on the cubic Schrödinger equation', J. Comput. Phys. 225, 284-299.

H. Berland, B. Owren and B. Skaflestad (2006), 'Solving the nonlinear Schrödinger equation using exponential integrators', Model. Identif. Control 27, 201-217.

H. Berland, B. Skaflestad and W. M. Wright (2007b), 'EXPINT: A MATLAB package for exponential integrators', ACM Trans. Math. Software 33, 4:1$4: 17$.

G. Beylkin, J. M. Keiser and L. Vozovoi (1998), 'A new class of time discretization schemes for the solution of nonlinear PDEs', J. Comput. Phys. 147, 362-387.

J. J. Biesiadecki and R. D. Skeel (1993), 'Dangers of multiple time step methods', J. Comput. Phys. 109, 318-328.

S. Blanes, F. Casas and J. Ros (2002), 'High order optimized geometric integrators for linear differential equations', BIT 42, 262-284.

S. Blanes, F. Casas, J. Oteo and J. Ros (2009), 'The Magnus expansion and some of its applications', Physics Reports 470, 151-238.

M. Born and V. Fock (1928), 'Beweis des Adiabatensatzes', Z. Phys. A Hadrons and Nuclei 51, 165-180.

M. A. Botchev, I. Faragó and R. Horváth (2009), 'Application of operator splitting to the Maxwell equations including a source term', Appl. Numer. Math. 59, 522-541.

M. A. Botchev, D. Harutyunyan and J. J. W. van der Vegt (2006), 'The Gautschi time stepping scheme for edge finite element discretizations of the Maxwell equations', J. Comput. Phys. 216, 654-686.

C. Budd and A. Iserles (1999), 'On the solution of linear differential equations in Lie groups', Philos. Trans. Royal Soc. A 357, 946-956.

M. Caliari (2007), 'Accurate evaluation of divided differences for polynomial interpolation of exponential propagators', Computing 80, 189-201.

M. Caliari and A. Ostermann (2009), 'Implementation of exponential Rosenbrocktype integrators', Appl. Numer. Math. 59, 568-581. 
M. Caliari, M. Vianello and L. Bergamaschi (2004), 'Interpolating discrete advection-diffusion propagators at Leja sequences', J. Comput. Appl. Math. 172, 79-99.

M. P. Calvo and C. Palencia (2006), 'A class of explicit multistep exponential integrators for semilinear problems', Numer. Math. 102, 367-381.

E. Celledoni, D. Cohen and B. Owren (2008), 'Symmetric exponential integrators with an application to the cubic Schrödinger equation', Found. Comp. Math. 8, 303-317.

E. Celledoni, A. Marthinsen and B. Owren (2003), 'Commutator-free Lie group methods', Future Generation Computer Systems 19, 341-352.

J. Certaine (1960), The solution of ordinary differential equations with large time constants. In Mathematical Methods for Digital Computers, Wiley, pp. 128 132.

D. Cohen, T. Jahnke, K. Lorenz and C. Lubich (2006), Numerical integrators for highly oscillatory Hamiltonian systems: A review. In Analysis, Modeling and Simulation of Multiscale Problems (A. Mielke, ed.), Springer, pp. 553-576.

M. Condon, A. Deaño and A. Iserles (2009), 'On highly oscillatory problems arising in electronic engineering', Mathematical Modelling and Numerical Analysis 43, 785-804.

S. M. Cox and P. C. Matthews (2002), 'Exponential time differencing for stiff systems', J. Comput. Phys. 176, 430-455.

H. De la Cruz, R. J. Biscay, F. Carbonell, T. Ozaki and J. Jimenez (2007), 'A higher order local linearization method for solving ordinary differential equations', Appl. Math. Comput. 185, 197-212.

P. Deuflhard (1979), 'A study of extrapolation methods based on multistep schemes without parasitic solutions', Z. Angew. Math. Phys. 30, 177-189.

P. Deuflhard, J. Hermans, B. Leimkuhler, A. Mark, S. Reich and R. D. Skeel, eds (1999), Algorithms for Macromolecular Modelling, Vol. 4 of Lecture Notes in Computational Science and Engineering, Springer.

J. Dixon and S. McKee (1986), 'Weakly singular discrete Gronwall inequalities', Z. Angew. Math. Mech. 66, 535-544.

V. L. Druskin and L. A. Knizhnerman (1991), 'Error bounds in the simple Lanczos procedure for computing functions of symmetric matrices and eigenvalues', Comput. Math. Math. Phys. 31, 20-30.

V. L. Druskin and L. A. Knizhnerman (1994), 'On application of the Lanczos method to solution of some partial differential equations', J. Comput. Appl. Math. 50, 255-262.

V. L. Druskin and L. A. Knizhnerman (1995), 'Krylov subspace approximation of eigenpairs and matrix functions in exact and computer arithmetic', Numer. Linear Algebra Appl. 2, 205-217.

B. L. Ehle and J. D. Lawson (1975), 'Generalized Runge-Kutta processes for stiff initial-value problems', J. Inst. Math. Appl. 16, 11-21.

M. Eiermann and O. G. Ernst (2006), 'A restarted Krylov subspace method for the evaluation of matrix functions', SIAM J. Numer. Anal. 44, 2481-2504.

E. Emmrich (2005), 'Stability and error of the variable two-step BDF for semilinear parabolic problems', J. Appl. Math. Comput. 19, 33-55. 
K.-J. Engel and R. Nagel (2000), One-Parameter Semigroups for Linear Evolution Equations, Vol. 194 of Graduate Texts in Mathematics, Springer.

A. Friedli (1978), Verallgemeinerte Runge-Kutta Verfahren zur Lösung steifer Differentialgleichungssysteme. In Numerical Treatment of Differential Equations (R. Burlirsch, R. Grigorieff and J. Schröder, eds), Vol. 631 of Lecture Notes in Mathematics, Springer, pp. 35-50.

R. A. Friesner, L. S. Tuckerman, B. C. Dornblaser and T. V. Russo (1989), 'A method for exponential propagation of large systems of stiff nonlinear differential equations', J. Sci. Comput. 4, 327-354.

A. Frommer and V. Simoncini (2008), Matrix functions. In Model Order Reduction: Theory, Research Aspects and Applications (W. H. Schilders and H. A. van der Vorst, eds), Mathematics in Industry, Springer, pp. 275-304.

E. Gallopoulos and Y. Saad (1992), 'Efficient solution of parabolic equations by Krylov approximation methods', SIAM J. Sci. Statist. Comput. 13, 12361264 .

B. García-Archilla, J. M. Sanz-Serna and R. D. Skeel (1998), 'Long-time-step methods for oscillatory differential equations', SIAM J. Sci. Comput. 20, 930-963.

W. Gautschi (1961), 'Numerical integration of ordinary differential equations based on trigonometric polynomials', Numer. Math. 3, 381-397.

M. A. Gondal (2010), 'Exponential Rosenbrock integrators for option pricing', J. Comput. Appl. Math. 234, 1153-1160.

C. González and M. Thalhammer (2007), 'A second-order Magnus-type integrator for quasi-linear parabolic problems', Math. Comp. 76, 205-231.

C. González, A. Ostermann and M. Thalhammer (2006), 'A second-order Magnustype integrator for nonautonomous parabolic problems', J. Comput. Appl. Math. 189, 142-156.

V. Grimm (2002), Exponentielle Integratoren als Lange-Zeitschritt-Verfahren für oszillatorische Differentialgleichungen zweiter Ordnung. Dissertation, Heinrich-Heine Universität Düsseldorf.

V. Grimm (2005a), 'A note on the Gautschi-type method for oscillatory secondorder differential equations', Numer. Math. 102, 61-66.

V. Grimm (2005b), 'On error bounds for the Gautschi-type exponential integrator applied to oscillatory second-order differential equations', Numer. Math. 100, 71-89.

V. Grimm and M. Hochbruck (2006), 'Error analysis of exponential integrators for oscillatory second-order differential equations', J. Phys. A 39, 5495-5507.

V. Grimm and M. Hochbruck (2008), 'Rational approximation to trigonometric operators', BIT 48, 215-229.

H. Grubmüller, H. Heller, A. Windemuth and K. Schulten (1991), 'Generalized Verlet algorithm for efficient molecular dynamics simulations with long-range interactions', Molecular Simulation 6, 121-142.

E. Hairer and C. Lubich (2000), 'Long-time energy conservation of numerical methods for oscillatory differential equations', SIAM J. Numer. Anal. 38, 414-441.

E. Hairer and C. Lubich (2009), Oscillations over long times in numerical Hamiltonian systems. In Highly Oscillatory Problems (E. H. B. Engquist, A. Fokas and A. Iserles, eds), Vol. 366 of London Mathematical Society Lecture Notes, Cambridge University Press, pp. 1-24. 
E. Hairer and G. Wanner (1996), Solving Ordinary Differential Equations II: Stiff and Differential-Algebraic Problems, Vol. 14 of Springer Series in Computational Mathematics, 2nd edn, Springer.

E. Hairer, C. Lubich and G. Wanner (2006), Geometric Numerical Integration, Structure-Preserving Algorithms for Ordinary Differential Equations, Vol. 31 of Springer Series in Computational Mathematics, Springer.

E. Hairer, S. P. Nørsett and G. Wanner (1993), Solving Ordinary Differential Equations I: Nonstiff Problems, Vol. 8 of Springer Series in Computational Mathematics, 2nd edn, Springer.

D. Henry (1981), Geometric Theory of Semilinear Parabolic Equations, Vol. 840 of Lecture Notes in Mathematics, Springer.

J. Hersch (1958), 'Contribution à la méthode des équations aux différences', Z. Angew. Math. Phys. 9, 129-180.

N. J. Higham (2008), Functions of Matrices: Theory and Computation, SIAM.

N. J. Higham and A. H. Al-Mohy (2010), Computing matrix functions. In Acta Numerica, Vol. 19, Cambridge University Press, pp. 159-208.

M. Hochbruck and C. Lubich (1997), 'On Krylov subspace approximations to the matrix exponential operator', SIAM J. Numer. Anal. 34, 1911-1925.

M. Hochbruck and C. Lubich (1999a), A bunch of time integrators for quantum/classical molecular dynamics. In Deuflhard et al. (1999), pp. 421-432.

M. Hochbruck and C. Lubich (1999b), 'Exponential integrators for quantumclassical molecular dynamics', BIT 39, 620-645.

M. Hochbruck and C. Lubich (1999c), 'A Gautschi-type method for oscillatory second-order differential equations', Numer. Math. 83, 403-426.

M. Hochbruck and C. Lubich (2003), 'On Magnus integrators for time-dependent Schrödinger equations', SIAM J. Numer. Anal. 41, 945-963.

M. Hochbruck and A. Ostermann (2005a), 'Explicit exponential Runge-Kutta methods for semilinear parabolic problems', SIAM J. Numer. Anal. 43, 10691090.

M. Hochbruck and A. Ostermann (2005b), 'Exponential Runge-Kutta methods for parabolic problems', Appl. Numer. Math. 53, 323-339.

M. Hochbruck and A. Ostermann (2006), 'Explicit integrators of Rosenbrock-type', Oberwolfach Reports 3, 1107-1110.

M. Hochbruck and J. van den Eshof (2006), 'Preconditioning Lanczos approximations to the matrix exponential', SIAM J. Sci. Comput. 27, 1438-1457.

M. Hochbruck, M. Hönig and A. Ostermann (2009a), 'A convergence analysis of the exponential Euler iteration for nonlinear ill-posed problems', Inverse Problems 25, 075009.

M. Hochbruck, M. Hönig and A. Ostermann (2009b), 'Regularization of nonlinear ill-posed problems by exponential integrators', Mathematical Modelling and Numerical Analysis 43, 709-720.

M. Hochbruck, C. Lubich and H. Selhofer (1998), 'Exponential integrators for large systems of differential equations', SIAM J. Sci. Comput. 19, 1552-1574.

M. Hochbruck, A. Ostermann and J. Schweitzer (2009c), 'Exponential Rosenbrocktype methods', SIAM J. Numer. Anal. 47, 786-803.

W. Hundsdorfer and J. G. Verwer (2007), Numerical Solution of Time-Dependent Advection-Diffusion-Reaction Equations, Vol. 33 of Springer Series in Computational Mathematics, corrected 2nd printing, Springer. 
K. J. In't Hout and J. A. C. Weideman (2009), Appraisal of a contour integral method for the Black-Scholes and Heston equations. Technical report, Department of Mathematics and Computer Science, University of Antwerp.

A. Iserles $(2002 a)$, 'On the global error of discretization methods for highlyoscillatory ordinary differential equations', BIT 42, 561-599.

A. Iserles (2002b), 'Think globally, act locally: Solving highly-oscillatory ordinary differential equations', Appl. Numer. Math. 43, 145-160.

A. Iserles and S. P. Nørsett (1999), 'On the solution of linear differential equations in Lie groups', Philos. Trans. Royal Soc. A 357, 983-1019.

A. Iserles and S. Nørsett (2004), 'On quadrature methods for highly oscillatory integrals and their implementation', BIT 44, 755-772.

A. Iserles, H. Z. Munthe-Kaas, S. P. Nørsett and A. Zanna (2000), Lie-group methods. In Acta Numerica, Vol. 9, Cambridge University Press, pp. 215365.

J. A. Izaguirre, S. Reich and R. D. Skeel (1999), 'Longer time steps for molecular dynamics', J. Chem. Phys. 110, 9853-9864.

T. Jahnke (2003), Numerische Verfahren für fast adiabatische Quantendynamik. $\mathrm{PhD}$ thesis, Eberhard-Karls-Universität, Tübingen, Germany.

T. Jahnke (2004), 'Long-time-step integrators for almost-adiabatic quantum dynamics', SIAM J. Sci. Comput. 25, 2145-2164.

T. Jahnke and C. Lubich (2000), 'Error bounds for exponential operator splittings', BIT 40, 735-744.

T. Jahnke and C. Lubich (2003), 'Numerical integrators for quantum dynamics close to the adiabatic limit', Numer. Math. 94, 289-314.

C. Karle, J. Schweitzer, M. Hochbruck and K.-H. Spatschek (2008), 'A parallel implementation of a two-dimensional fluid laser-plasma integrator for stratified plasma-vacuum systems', J. Comput. Phys. 227, 7701-7719.

C. Karle, J. Schweitzer, M. Hochbruck, E. W. Laedke and K.-H. Spatschek (2006), 'Numerical solution of nonlinear wave equations in stratified dispersive media', J. Comput. Phys. 216, 138-152.

A.-K. Kassam and L. N. Trefethen (2005), 'Fourth-order time-stepping for stiff PDEs', SIAM J. Sci. Comput. 26, 1214-1233.

C. Klein (2008), 'Fourth order time-stepping for low dispersion Korteweg-de Vries and nonlinear Schrödinger equations', Electron. Trans. Numer. Anal. 29, 116135.

L. A. Knizhnerman (1991), 'Computation of functions of unsymmetric matrices by means of Arnoldi's method', J. Comput. Math. Math. Phys. 31, 5-16 (in the Russian issue).

L. A. Knizhnerman (1992), 'Error bounds in Arnoldi's method: The case of a normal matrix', Comput. Math. Math. Phys. 32, 1199-1211.

L. Knizhnerman and V. Simoncini (2009), 'A new investigation of the extended Krylov subspace method for matrix function evaluations', Numer. Linear Algebra Appl. In press.

R. Kosloff (1994), 'Propagation methods for quantum molecular dynamics', Annu. Rev. Phys. Chem. 45, 145-178.

S. Krogstad (2005), 'Generalized integrating factor methods for stiff PDEs', J. Comput. Phys. 203, 72-88. 
J. D. Lambert and S. T. Sigurdsson (1972), 'Multistep methods with variable matrix coefficients', SIAM J. Numer. Anal. 9, 715-733.

J. D. Lawson (1967), 'Generalized Runge-Kutta processes for stable systems with large Lipschitz constants', SIAM J. Numer. Anal. 4, 372-380.

H. Lee and D. Sheen (2009), 'Laplace transformation method for the Black-Scholes equations', Int. J. Numer. Anal. Model. 6, 642-659.

M. López-Fernández (2009), On the implementation of exponential methods for semilinear parabolic equations. Technical report, Instituto de Ciencias Matemáticas, Madrid, Spain.

M. López-Fernández, C. Palencia and A. Schädle (2006), 'A spectral order method for inverting sectorial Laplace transforms', SIAM J. Numer. Anal. 44, 13321350 .

K. Lorenz, T. Jahnke and C. Lubich (2005), 'Adiabatic integrators for highly oscillatory second-order linear differential equations with time-varying eigendecomposition', BIT 45, 91-115.

C. Lubich (2008), From Quantum to Classical Molecular Dynamics: Reduced Models and Numerical Analysis, Zurich Lectures in Advanced Mathematics, European Mathematical Society (EMS).

A. Lunardi (1995), Analytic Semigroups and Optimal Regularity in Parabolic Problems, Vol. 16 of Progress in Nonlinear Differential Equations and their Applications, Birkhäuser.

Q. Ma and J. A. Izaguirre (2003a), Long time step molecular dynamics using targeted Langevin stabilization. In SAC '03: Proc. 2003 ACM Symposium on Applied Computing, ACM, New York, pp. 178-182.

Q. Ma and J. A. Izaguirre (2003b), 'Targeted mollified impulse: A multiscale stochastic integrator for long molecular dynamics simulations', Multiscale Model. Simul. 2, 1-21.

Q. Ma, J. A. Izaguirre and R. D. Skeel (2003), 'Verlet-I/R-RESPA/impulse is limited by nonlinear instabilities', SIAM J. Sci. Comput. 24, 1951-1973.

W. Magnus (1954), 'On the exponential solution of differential equations for a linear operator', Comm. Pure Appl. Math. 7, 649-673.

A. Martínez, L. Bergamaschi, M. Caliari and M. Vianello (2009), 'A massively parallel exponential integrator for advection-diffusion models', J. Comput. Appl. Math. 231, 82-91.

R. I. McLachlan and G. R. W. Quispel (2002), Splitting methods. In Acta Numerica, Vol. 11, Cambridge University Press, pp. 341-434.

B. V. Minchev and W. Wright (2005), A review of exponential integrators for first order semi-linear problems. Preprint, NTNU Trondheim.

P. C. Moan and J. Niesen (2008), 'Convergence of the Magnus series', Found. Comput. Math. 8, 291-301.

C. Moler and C. Van Loan (2003), 'Nineteen dubious ways to compute the exponential of a matrix, twenty-five years later', SIAM Rev. 45, 3-49.

I. Moret and P. Novati (2001), 'An interpolatory approximation of the matrix exponential based on Faber polynomials', J. Comput. Appl. Math. 131, 361380 .

I. Moret and P. Novati (2004), 'RD-rational approximations of the matrix exponential', BIT 44, 595-615. 
A. Nauts and R. E. Wyatt (1983), 'New approach to many-state quantum dynamics: The recursive-residue-generation method', Phys. Rev. Lett. 51, 22382241 .

P. Nettesheim and C. Schütte (1999), Numerical integrators for quantum-classical molecular dynamics. In Deuflhard et al. (1999), pp. 396-411.

P. Nettesheim, F. A. Bornemann, B. Schmidt and C. Schütte (1996), 'An explicit and symplectic integrator for quantum-classical molecular dynamics', Chem. Phys. Lett. 256, 581-588.

J. Niegemann, L. Tkeshelashvili and K. Busch (2007), 'Higher-order time-domain simulations of Maxwell's equations using Krylov-subspace methods', J. Comput. Theor. Nanoscience 4, 627-634.

J. Niehoff (2007), Projektionsverfahren zur Approximation von Matrixfunktionen mit Anwendungen auf die Implementierung exponentieller Integratoren. Dissertation, Heinrich-Heine Universität Düsseldorf, Mathematisches Institut.

J. Niesen and W. Wright (2009), A Krylov subspace algorithm for evaluating the $\varphi$ functions appearing in exponential integrators. Preprint: arXiv:0907.4631v1.

S. P. Nørsett (1969), An A-stable modification of the Adams-Bashforth methods. In Conference on the Numerical Solution of Differential Equations, Vol. 109 of Lecture Notes in Mathematics, Springer, pp. 214-219.

A. Ostermann, M. Thalhammer and W. M. Wright (2006), 'A class of explicit exponential general linear methods', BIT 46, 409-431.

T. J. Park and J. C. Light (1986), 'Unitary quantum time evolution by iterative Lanczos reduction', J. Chem. Phys. 85, 5870-5876.

A. Pazy (1992), Semigroups of Linear Operators and Applications to Partial Differential Equations, Vol. 44 of Applied Mathematical Sciences, corrected 2nd printing, Springer.

U. Peskin, R. Kosloff and N. Moiseyev (1994), 'The solution of the time dependent Schrödinger equation by the $\left(t, t^{\prime}\right)$ method: The use of global polynomial propagators for time dependent Hamiltonians', J. Chem. Phys. 100, 8849-8855.

D. A. Pope (1963), 'An exponential method of numerical integration of ordinary differential equations', Comm. Assoc. Comput. Mach. 6, 491-493.

M. Pototschnig, J. Niegemann, L. Tkeshelashvili and K. Busch (2009), 'Timedomain simulations of nonlinear Maxwell equations using operator-exponential methods', IEEE Trans. Antenn. Propag. 57, 475-483.

N. Rambeerich, D. Y. Tangman, A. Gopaul and M. Bhuruth (2009), 'Exponential time integration for fast finite element solutions of some financial engineering problems', J. Comput. Appl. Math. 224, 668-678.

J. I. Ramos and C. M. García-López (1997), 'Piecewise-linearized methods for initial-value problems', Appl. Math. Comput. 82, 273-302.

Y. Saad (1992), 'Analysis of some Krylov subspace approximations to the matrix exponential operator', SIAM J. Numer. Anal. 29, 209-228.

Y. Saad (1994), SPARSKIT: A basic tool kit for sparse matrix computations, version 2. Technical report, Department of Computer Science and Engineering, University of Minnesota.

Y. Saad (2003), Iterative Methods for Sparse Linear Systems, 2nd edn, SIAM.

A. Schädle, M. López-Fernández and C. Lubich (2006), 'Fast and oblivious convolution quadrature', SIAM J. Sci. Comput. 28, 421-438. 
T. Schlick, R. D. Skeel, A. T. Brunger, L. V. Kalé, J. A. Board, J. Hermans and K. Schulten (1999), 'Algorithmic challenges in computational molecular biophysics', J. Comput. Phys. 151, 9-48.

T. Schmelzer and L. N. Trefethen (2007), 'Evaluating matrix functions for exponential integrators via Carathéodory-Fejér approximation and contour integrals', Electron. Trans. Numer. Anal. 29, 1-18.

C. Schütte and F. A. Bornemann (1999), 'On the singular limit of the quantumclassical molecular dynamics model', SIAM J. Appl. Math. 59, 1208-1224.

R. B. Sidje (1998), 'Expokit: A software package for computing matrix exponentials', ACM Trans. Math. Software 24, 130-156.

D. E. Stewart and T. S. Leyk (1996), 'Error estimates for Krylov subspace approximations of matrix exponentials', J. Comput. Appl. Math. 72, 359-369.

K. Strehmel and R. Weiner (1987), 'B-convergence results for linearly implicit one step methods', BIT 27, 264-281.

K. Strehmel and R. Weiner (1992), Linear-implizite Runge-Kutta Methoden und ihre Anwendungen, Vol. 127 of Teubner-Texte zur Mathematik, Teubner.

H. Tal-Ezer and R. Kosloff (1984), 'An accurate and efficient scheme for propagating the time-dependent Schrödinger equation', J. Chem. Phys. 81, 3967-3971.

H. Tal-Ezer, R. Kosloff and C. Cerjan (1992), 'Low-order polynomial approximation of propagators for the time-dependent Schrödinger equation', J. Comput. Phys. 100, 179-187.

D. Y. Tangman, A. Gopaul and M. Bhuruth (2008), 'Exponential time integration and Chebychev discretisation schemes for fast pricing of options', Appl. Numer. Math. 58, 1309-1319.

U. Tautenhahn (1994), 'On the asymptotical regularization of nonlinear ill-posed problems', Inverse Problems 10, 1405-1418.

S. Teufel (2003), Adiabatic Perturbation Theory in Quantum Dynamics, Vol. 1821 of Lecture Notes in Mathematics, Springer.

M. Thalhammer (2006), 'A fourth-order commutator-free exponential integrator for nonautonomous differential equations', SIAM J. Numer. Anal. 44, 851864 .

M. Tokman (2006), 'Efficient integration of large stiff systems of ODEs with exponential propagation iterative (EPI) methods', J. Comput. Phys. 213, 748-776.

M. Tokman and P. M. Bellan (2002), 'Three-dimensional model of the structure and evolution of coronal mass ejections', Astrophys. J. 567, 1202-1210.

L. N. Trefethen, J. A. C. Weideman and T. Schmelzer (2006), 'Talbot quadratures and rational approximations', BIT 46, 653-670.

M. Tuckerman, B. J. Berne and G. J. Martyna (1992), 'Reversible multiple time scale molecular dynamics', J. Chem. Phys. 97, 1990-2001.

J. Verwer (1976), 'On generalized linear multistep methods with zero-parasitic roots and an adaptive principal root', Numer. Math. 27, 143-155.

J. G. Verwer and M. A. Botchev (2009), 'Unconditionally stable integration of Maxwell's equations', Linear Algebra Appl. 431, 300-317. 